\title{
Yeast ATM and ATR use different mechanisms to spread histone H2A phosphorylation around a DNA double-strand break
}

\author{
Kevin $\mathrm{Li}^{1}$, Gabriel Bronk ${ }^{1}$, Jane Kondev ${ }^{1, *}$ and James E. Haber ${ }^{2, *}$ \\ ${ }^{1}$ Department of Physics, Brandeis University, Waltham, MA \\ ${ }^{2}$ Department of Biology and Rosenstiel Basic Medical Sciences Research Center, Brandeis \\ University, Waltham, MA \\ * to whom correspondence should be addressed \\ haber@brandeis.edu, kondev@brandeis.edu \\ Author Contributions: K.L, G.B, J.K, and J.E.H designed research. K.L. performed \\ experiments. G.B. and K.L. analyzed the data. G.B. performed mathematical modelling \\ and simulations. K.L, G.B, J.K, and J.E.H wrote the paper.
}

Competing Interest Statement: The authors declare no conflict of interest 


\begin{abstract}
One of the hallmarks of DNA damage is the rapid spreading of phosphorylated histone H2A $(\gamma-\mathrm{H} 2 \mathrm{AX})$ around a DNA double-strand break (DSB). In the budding yeast $S$. cerevisiae, nearly all $\mathrm{H} 2 \mathrm{~A}$ isoforms can be phosphorylated, either by Mec1 ${ }^{\mathrm{ATR}}$ or Tel1 ${ }^{\mathrm{ATM}}$ checkpoint kinases. We induced a site-specific DSB with HO endonuclease at the MAT locus on chromosome III and monitored the formation of $\gamma-\mathrm{H} 2 \mathrm{AX}$ by ChIP-qPCR in order to uncover the mechanisms by which $\mathrm{Mec} 1^{\mathrm{ATR}}$ and Tel1 ${ }^{\mathrm{ATM}}$ propagate histone modifications across chromatin. With either kinase, $\gamma-\mathrm{H} 2 \mathrm{AX}$ spreads as far as $\sim 50 \mathrm{~kb}$ on both sides of the lesion within $1 \mathrm{~h}$; but the kinetics and distribution of modification around the DSB are significantly different. The total accumulation of phosphorylation is reduced by about half when either of the two H2A genes is mutated to the nonphosphorylatable S129A allele. Mec1 activity is limited by the abundance of its ATRIP partner, Ddc2. Moreover, Mec1 is more efficient than Tel1 at phosphorylating chromatin in trans - at distant undamaged sites that are brought into physical proximity to the DSB. We compared experimental data to mathematical models of spreading mechanisms to determine whether the kinases search for target nucleosomes by primarily moving in three dimensions through the nucleoplasm or in one dimension along the chromatin. Bayesian model selection indicates that Mec1 primarily uses a 3D diffusive mechanism, whereas Tell undergoes directed motion along the chromatin.
\end{abstract}

\title{
Introduction
}

Genetic loci can be separated by thousands to millions of base pairs in the nucleus, constraining contact between distant regions of the genome. Yet many biological processes rely on the effective communication between distant parts of the genome to facilitate a diverse range of phenomena such as the regulation of gene expression through promoterenhancer interactions, the formation of chromatin loops during chromosome condensation or the initiation of homologous recombination in the presence of DNA breaks (1-3). Therefore, an understanding of many nuclear processes can be uncovered by investigating how distant chromosomal regions can establish genomic interactions in a timely fashion (4).

Communication between remote parts of the genome is often facilitated by the presence of intermediary proteins that shuttle information in a three-dimensional (3D) manner through the nucleoplasm or in a one-dimensional (1D) manner along the chromatin. 3D modes of communication lead to both intra- and interchromosomal interactions either by the physical folding of chromatin to bring two regions of the genome within close proximity (looping) or by $3 \mathrm{D}$ diffusion of a protein from one genetic locus to another through the surrounding nucleoplasm (5). In both looping and 3D diffusion, information can be transferred over many kilobases without having to interact with the intervening chromatin. In contrast, 1D mechanisms are intrinsically intrachromosomal and communication is predicated on the movement of DNA-bound proteins along the contours of the chromatin fiber. 1D mechanisms include either diffusive or unidirectional motion of proteins along the chromatin (5). Comparisons of experimental data with biophysical models of chromatin looping, 3D diffusion, 1D diffusion and directed sliding provides insight into how specific information transfers take place in the nucleus. 
In particular, we are interested in studying how genomic interactions are achieved over a distance of many kilobase pairs $(\mathrm{kb})$ after DNA damage. The rapid formation of $\gamma-\mathrm{H} 2 \mathrm{AX}$, the phosphorylated form of histone $\mathrm{H} 2 \mathrm{~A}$ (H2AX in mammals), over an extensive region of the chromatin is one indicator of DNA double strand breaks (DSBs) (6-9). In budding yeast, $\gamma-\mathrm{H} 2 \mathrm{AX}$ is formed by the phosphorylation at H2A-S129 by the checkpoint kinases Mec1 and Tel1, homologs of mammalian ATR and ATM, respectively. Previous studies have shown that Mec1 and Tell are both capable of forming $\gamma-\mathrm{H} 2 \mathrm{AX}$ regions up to $50 \mathrm{~kb}$ on either side of a DSB (9), while their mammalian homologs, especially ATM, are able to phosphorylate histones in chromatin regions in excess of $1 \mathrm{Mb}$ in mammalian cells $(6$, 7). $\gamma-\mathrm{H} 2 \mathrm{AX}$ has been shown to play a role in the recruitment and retention of factors responsible for efficient DNA repair, DNA damage signaling and chromatin remodeling, but is not essential for these processes $(8,9)$.

Mec1 and Tel1 are activated in the presence of a DSB by different mechanisms. In budding yeast, as in mammals, Tel1 ${ }^{\mathrm{ATM}}$ is attracted to a broken chromosome end by its association with Mre11-Rad50-Xrs2 ${ }^{\mathrm{Nbs} 1}$ (13). In contrast, the recruitment of $\mathrm{Mec}^{\mathrm{ATR}}$ requires that the broken end undergoes some 5'to 3' resection, promoting the binding of the single-strand DNA-binding protein complex, RPA. RPA is then bound by Ddc $2^{\text {ATRIP }}$, which is the obligate partner of $\operatorname{Mec}^{\text {ATR }}(14,15)$. While we know that both Mec1 and Tell are actively recruited to DSBs and are involved in $\gamma-\mathrm{H} 2 \mathrm{AX}$ formation, the means by which Mec1 and Tel1 kinases reach histones tens of kilobases from the DSB is still unknown. To address this problem, we monitored the kinetics and extent of $\gamma$-H2AX formation in budding yeast, by ChIP-qPCR, after creating a synchronously-induced DSB at a specific location on chromosome III. We compared experimentally measured $\gamma-\mathrm{H} 2 \mathrm{AX}$ distributions to mathematical formulations of different phosphorylation spreading mechanisms to determine whether the kinases can reach histones far from the DSB using chromatin looping, 3D diffusive, 1D diffusive or directed sliding mechanisms. Through Bayesian model selection, we conclude that Mec1 reaches target histones by a $3 \mathrm{D}$ diffusive mechanism, while Tell dynamics is best described by directed 1D sliding along the chromatin.

\section{Results}

\section{Mec1 and Tel1 kinases use different mechanisms for $\gamma$-H2AX spreading}

We studied phosphorylation spreading in S. cerevisiae after inducing a site-specific DSB using a galactose-inducible $\mathrm{HO}$ endonuclease, resulting in robust cleavage at the $M A T$ locus on chromosome III within 30 min (Figure S1). DSBs were rendered irreparable by homologous recombination through the deletion of both $H M L$ and $H M R$ donor loci (16). $\gamma$-H2AX spreading was studied in G1-arrested haploid MATa cells where the re-ligation of cleaved ends by nonhomologous end-joining was prevented by deleting NEJ1 or YKU80 (17). The formation of $\gamma-\mathrm{H} 2 \mathrm{AX}$ over a region of roughly $50 \mathrm{~kb}$ on both sides of the DSB was quantified by ChIP-qPCR with an antibody specific to phosphorylated H2A-S129. To differentiate between the mechanisms used by $\mathrm{Mecl}$ and Tell to phosphorylate large regions of chromatin adjacent to the break, we measured $\gamma-\mathrm{H} 2 \mathrm{AX}$ levels in strains where both Mec1 and Tel1 were active or when only Mec1 or Tell kinase was active. 
In G1-arrested cells, 5' to 3' resection of DSB ends is blocked $(12,13)$. Under these conditions, the absence of RPA binding leads to lack of Mec1 recruitment; so only Tell is active (9). We confirmed that in nej1s G1-arrested cells, Tell robustly modifies the chromatin adjacent to $M A T$, but there is only a very small signal in strains where only Mec1 is present; the observed signal likely reflects the small fraction of cells that have escaped $\alpha$-factor arrest (Figures 1A and 1B).

To compare Mec1 and Tel1 activity in G1 cells, we deleted YKU80, which normally blocks access of the exonuclease Exo1 to the DSB (20). $y k u 80 \Delta$ cells generate sufficient ssDNA to recruit the single-strand binding protein complex RPA and activate Mec1-Ddc2 (14). Under these conditions, both Mecl alone or Tell alone were efficient at phosphorylating histone H2A (Figures 1D and 1E). In the absence of $Y K U 80$, the Tell-only derivative exhibited a significant increase in $\gamma-\mathrm{H} 2 \mathrm{AX}$ levels over background by $25 \mathrm{~min}$ (Figure 1E), when about $60 \%$ of the MAT locus had been cleaved (Figure S1). The appearance of $\gamma-$ $\mathrm{H} 2 \mathrm{AX}$ in the Mec1-only strain was slightly delayed by about $5 \mathrm{~min}$ (Figure 1D). In both cases, the extent of phosphorylation was largely complete by $75 \mathrm{~min}$, but with noticeable differences between the phosphorylation profiles of Mec1 and Tel1. Although Tel1 appears to be more rapidly activated, the extent of $\gamma-\mathrm{H} 2 \mathrm{AX}$ spreading for Tel1 is more confined to the region adjacent to the break than Mec1. The difference in the profiles of modification can be shown by calculating the mean modification distance (MMD), the distance from the break that encompasses half the $\gamma$-H2AX profile. At $75 \mathrm{~min}$, the $95 \%$ confidence interval (CI) for the MMD of Mec1 is MMD Mecl $=[12.9,14.5] \mathrm{kb}$ while the $95 \%$ CI for the MMD of Tel1 is $\mathrm{MMD}_{\mathrm{Tel} 1}=[10.8,11.6] \mathrm{kb}$, indicating that the activities of Mec1 and Tel1 lead to significantly different profiles of spreading (Figure S2A).

When both kinases are active, the kinetics of phosphorylation were more rapid and reached an apparent steady state around $45 \mathrm{~min}$, suggesting that both $\mathrm{Mec} 1$ and Tel1 participate in phosphorylating H2A (Figures $1 \mathrm{C}$ and $1 \mathrm{~F}$ ). We also note that at later time points, the level of modification close to the DSB does not increase, most likely due to loss of nucleosomes during 5' to 3' resection that displaces histones, at a rate of $4 \mathrm{~kb} / \mathrm{hr}(21)$. Although the $\gamma$ $\mathrm{H} 2 \mathrm{AX}$ profiles for each kinase alone or when both are active differ in their kinetics and extent of spreading, the total amount of $\gamma-\mathrm{H} 2 \mathrm{AX}$ formation is the same by $75 \mathrm{~min}$, suggesting that Mec1 and Tell have similar levels of phosphorylation activity, but distribute the $\gamma-\mathrm{H} 2 \mathrm{AX}$ sites differently (Figures $1 \mathrm{~F}$ and $\mathrm{S} 2 \mathrm{~B}$ ).

\section{The amount, but not the profile, of $\gamma-\mathrm{H} 2 \mathrm{AX}$ is affected by reducing the density of phosphorylation sites.}

To further investigate the differences between the Mec1 and Tell modes of spreading, we mutated the phosphorylation site of one or the other H2A gene. Using CRISPR/Cas9, we mutated either HTA1-S129 or HTA2-S129 to alanine (see Methods), so that the density of phosphorylatable H2A-S129 sites should be reduced by half. As expected, each of these mutations reduced the level of $\gamma-\mathrm{H} 2 \mathrm{AX}$ (Figures 2A, 2B and S2B) suggesting that the availability of $\mathrm{H} 2 \mathrm{~A}$ phosphorylation sites is limiting - i.e. the amount of $\gamma-\mathrm{H} 2 \mathrm{AX}$ formed depends on the density of phosphorylatable sites. Despite the reduction in $\gamma$-H2AX levels, the extent of phosphorylation spreading for $\mathrm{Mec} 1$ and Tel1 remain unchanged when only 
$50 \%$ the sites can be phosphorylated; moreover, the two mutant profiles were not significantly different from each other. The 95\% CI of the MMDs at $75 \mathrm{~min}$ for Mec1 are $\mathrm{MMD}_{\mathrm{Mec} 1, \mathrm{HTA} 1}=[13.2,14.4] \mathrm{kb}$ and $\mathrm{MMD}_{\mathrm{Mec} 1, \mathrm{HTA} 2}=[13.0,14.2] \mathrm{kb}$, while the $95 \% \mathrm{CI}$ of the MMDs for Tell are MMD ${ }_{\text {Tel1, } \mathrm{HTAl}}=[10.0,10.8] \mathrm{kb}$ and $\mathrm{MMD}_{\mathrm{Tel} 1, \mathrm{HTA} 2}=[10.7,11.9] \mathrm{kb}$ (Figures 2 and S2A).

\section{Mec1 modifies histones at distant chromosome sites that are recruited to the DSB}

We previously showed that a DSB near one centromere will lead to the modification of all the other 15 pericentromeric regions clustered at the spindle pole body. This modification is predominantly carried out by Mec1 and occurs at about 1/10 the magnitude as modifications on the broken chromosome (22). $\gamma$-H2AX is also weakly spread around the recombination enhancer, RE, a sequence near the $H M L$ locus and roughly $170 \mathrm{~kb}$ from $M A T$, that facilitates pairing between MATa and the $H M L$ donor (23). RE binds multiple copies of the Fkh1 protein whose FHA domain can presumably also bind to phosphothreonines that are generated near the DSB; however, the specific phosphorylated target of the FHA domain remains unknown. ChIP using an anti- $\gamma-\mathrm{H} 2 \mathrm{AX}$ antibody pulls down the RE region in MATa cells, when Fkh1 binds to RE, but not in MATa cells, when Fkh1 binding is repressed (24). Thus, a kinase originating at the MAT locus can only phosphorylate the region around RE by $3 \mathrm{D}$ diffusive or looping mechanisms, and only when it has been brought into proximity with the DSB. In our strains $H M L$ is deleted, but the Fkh1 proteins bound to RE are still able to interact with phosphorylated targets near $M A T$. At $75 \mathrm{~min}$, the level of ChIP-qPCR $10 \mathrm{~kb}$ away from RE was significantly increased above background for Mec1 (Figure 3A), while Tell did not show a significant increase at these distances, except possibly at the $-5 \mathrm{~kb}$ location (Figure $3 \mathrm{~B}$ ). Taken all together the experimental evidence suggests that Mecl is the kinase that is primarily responsible for phosphorylation spreading in trans.

\section{Biophysical models of chromatin-modification spreading can be used to determine the mechanism by which Mec1 and Tel1 phosphorylate distant H2As}

By comparing the phosphorylation data to mathematical models of phosphorylation spreading, we can infer the mechanisms by which the kinases spread $\gamma-\mathrm{H} 2 \mathrm{AX}$. We focused on two classes of models, one of which assumes that the spreading occurs by the kinase moving three-dimensionally through the nucleoplasm while the other assumes that the kinase moves one-dimensionally along the chromatin. For each class, we chose two minimal models commonly found in the literature (25): the 3D models are represented by chromatin looping and 3D diffusion while the 1D models are represented by 1D diffusion and directed sliding (Figures 4A-4D). (The models are described in detail in Computational Methods and Supplementary Information). All four models assume an initial recruitment of the kinases to the break site with a rate $k_{\text {init }}$ but differ thereafter. For the $1 \mathrm{D}$ models, the parameter $k_{\text {init }}$ comprises both the recruitment and detachment from the DSB to begin traversing the chromosome. Although each nucleosome contains two monomers of $\mathrm{H} 2 \mathrm{~A}$, in these models we treat each phosphorylation site as a separate entity.

Thermal fluctuations cause chromosomes to form dynamic conformations, including transient loops. In the looping model (Figure 4A), the kinase is tethered at the break end 
and folding of the chromatin brings distant $\mathrm{H} 2 \mathrm{As}$ into physical contact with the DSB-bound kinase. Using a worm-like chain model of the chromosome, we compute $P_{\text {loop }}(x, l)$, the probability of a looped conformation in which the DSB-bound kinase is in contact with an H2A located $x$ kilobases away, where $l$ is the Kuhn length of the chromatin. When the kinase is in contact with an $\mathrm{H} 2 \mathrm{~A}$, phosphorylation occurs at a rate $k_{\text {cat }}$.

The 3D diffusion model (Figure 4B) assumes that the kinase transiently binds to the DSB, where it is activated, and then released at a rate kleaveDSB. The activated kinase diffuses through the nucleoplasm until it encounters and phosphorylates an $\mathrm{H} 2 \mathrm{~A}$ site. For an $\mathrm{H} 2 \mathrm{~A}$ located $x$ kilobases from the DSB, we use the worm-like chain polymer model to calculate the mean 3D distance between this H2A and the DSB. This distance is used to compute the probability $P_{\text {contact }}(x, l)$ that the kinase will come into contact with the H2A.

In the 1D diffusion model (Figure 4C), we assume that the kinase lands at the DSB and proceeds to diffuse along the chromatin with the one-dimensional diffusion coefficient $D$. When it encounters a histone, the kinase phosphorylates the $\mathrm{H} 2 \mathrm{~A}$ at a rate $k_{\text {cat }}$. The directed sliding model (Figure 4D) is similar to 1D diffusion except that the kinase moves unidirectionally away from the DSB along the chromatin. The kinase slides onto adjacent histones at a rate $k_{\text {slide }}$ and phosphorylates all $\mathrm{H} 2 \mathrm{As}$ that it comes across until the kinase detaches from the chromatin at a rate $k_{o f f}$.

For an H2A located $x$ kilobases from the break site, each of our models predicts the probability $P(x, t)$ that the histone has been phosphorylated at time $t$ after the DSB induction. Each model predicts a distinct $P(x, t)$ allowing us to use the experimentally measured $\gamma-\mathrm{H} 2 \mathrm{AX}$ profiles to determine the best spreading models for Mec1 and Tell. However, before we can directly compare the theoretical predictions to the experimental measurements, it is necessary to convert the predicted probabilities $P(x, t)$ to the expected ChIP signals. We formulate a simple thermodynamic model for the ChIP pulldown to account for the binding of antibodies to a chromatin fragment containing multiple phosphorylated H2As (Figure 4E). Sonication during ChIP results in chromatin fragments of roughly $500 \mathrm{bp}$. We introduce the following model parameters: $N_{H 2 A}, f$ and $C$. The parameter $N_{H 2 A}$ represents the average number of H2As on the chromatin fragment, and $f$ is the probability that each $\gamma-\mathrm{H} 2 \mathrm{AX}$ is bound by an antibody; we assume that the $\gamma-\mathrm{H} 2 \mathrm{AX}-$ antibody interaction is independent of the other $\gamma$-H2AXs on the same chromatin fragment. We also make the assumption that the presence of one $\gamma-\mathrm{H} 2 \mathrm{AX}$-antibody interaction is sufficient for pulling down the entire chromatin fragment during ChIP. Finally, $C$ accounts for the loss in DNA recovery during the wash steps of ChIP. We derive the ChIP model in detail in the Supplementary Information.

\section{Bayes factors reveal the most likely phosphorylation spreading mechanisms for each kinase}

The best model for each kinase was determined by calculating the Bayes factor, which expresses how much less probable one model is compared to another model, given all the data collected in our experiments. When evaluating the Bayes factor, we take into account both the $\gamma-\mathrm{H} 2 \mathrm{AX}$ profiles around the DSB (Figures 1D and 1E) as well as the phosphorylation in trans near the RE locus (Figure 3). The results are recorded in Table 1 
as the $\log _{10}$ of the Bayes factor. Using Bayesian model selection, we determined that 3D diffusion is the most likely mechanism for Mec1, while directed sliding is the most likely mechanism for Tell.

We first computed the Bayes factors for Tel1 alone (Table 1A). It is clear that 1D models are vastly more likely than the 3D models for Te11. Therefore, we discarded the 3D models for Tel1. Next, we simultaneously computed the Bayes factors for both 1D models of Tel1 and either 1D or 3D models for Mec1 by imposing the constraint that the values of the ChIP-model parameters ( $C, f$ and $N_{H 2 A}$ ) must be the same regardless of which kinase is active. The simultaneous pairwise calculations of the Bayes factor for both Mec1 and Tel1 models (excluding the 3D models for Tel1) are shown in Table 1B. Further details of this calculation can be found in the Supplementary Information.

After determining the best models for Tel1 and Mec1, we implemented Bayesian parameter estimation to find the optimal values for the model parameters. When performing parameter estimation, the data in Figures 1D, 1E, 2A, 2B, 3A, and 3B were fit simultaneously to the best models. The best models are plotted using the optimal parameter values and are in quantitative agreement with the experimental data (Figure 5). All of the less likely models are plotted against data in Figures S3-S14.

In Figure 6A, we plot the predicted phosphorylation levels by Mec1 around RE. The 3D models predict that when RE is bound to $M A T$, the loci within $10 \mathrm{~kb}$ of RE will be in close proximity to the DSB and so the kinase will phosphorylate H2As at these loci. When modeling $\gamma$-H2AX spreading around RE, we include two additional parameters, which take into account the binding of RE to MAT (see Supplementary Information). We did not plot the predicted phosphorylation by Tell near RE because the directed sliding model predicts that RE is too far away from the DSB for there to be any phosphorylation by Tell above the background level.

\section{Theoretical predictions of phosphorylation spreading are in agreement with data from mutant strains in which $50 \%$ of the $\mathrm{H} 2 \mathrm{As}$ are phosphorylatable}

Next, we compared the measured $\gamma$-H2AX levels from the $50 \%$ phosphorylatable strains to the theoretical predictions of the 3D diffusion model for Mec1 and the directed sliding model for Tell (Figures 2, 6B and 6C), when only one out of every two H2As can be phosphorylated. For the $50 \%$ phosphorylatable strains, we used the same parameter values as those used to fit the wild-type H2A strains (Figure 5). The theoretical predictions for the $50 \%$ phosphorylatable strains are largely in agreement with the experimental data (Figures $6 \mathrm{~B}$ and $6 \mathrm{C})$.

While it was conceivable that the introduction of the S129A mutant allele might impact the sliding rate of Tel1, we find that, in fact, Tel1's sliding remains unaffected. We performed fits in which the HTA-S129A strains were allowed to have both $k_{\text {slide }}$ and $k_{\text {off }}$ differ from the parameter values established for the wildtype H2A strains. The sliding parameters remain unchanged in the HTA-S129A mutants with the optimal $k_{\text {slide }}$ and $k_{\text {off }}$ values being within $10 \%$ of the established rates for wildtype H2A strains. Moreover, the Bayes factor calculation tells us that the most likely directed sliding model for Tell is one in which $k_{\text {slide }}$ 
and $k_{\text {off }}$ are unaltered in the $50 \%$ phosphorylatable strains. From our Bayesian parameter estimation, the average distance over which Tel1 slides is $11 \mathrm{~kb}$ (95\% CI of 9.7-12 kb) before falling off the chromosome.

The agreement between theory and experiments on $H T A-S 129 A$ mutants also validates our model of the ChIP process, which provides us with a quantitative understanding of the relationship between the amount of $\gamma-\mathrm{H} 2 \mathrm{AX}$ on the chromatin and the DNA recovery from ChIP (Figure 4E). The recovery of $>50 \%$ of the ChIP signal when one H2A gene is rendered nonphosphorylatable (Figure S2B) is explained by the assumption that there is a substantial chance that a DNA fragment will be pulled down even if there is only one $\gamma-$ $\mathrm{H} 2 \mathrm{AX}$ on the $\sim 500 \mathrm{bp}$ DNA fragment (Figure 4E). Even though the H2A-S129A strains have half as many $\gamma-\mathrm{H} 2 \mathrm{AX}$ per fragment, there is still enough $\gamma-\mathrm{H} 2 \mathrm{AX}$ s per fragment to be recovered with ChIP (see Supplementary Information).

\section{Phosphorylation levels by Mec1 is improved by overexpressing its binding partner Ddc2.}

Previously Ddc2 overexpression was shown to increase Mec1's checkpoint activity, whereas increasing Mec1 expression had no consequence (26). We therefore asked if the efficiency of $\mathrm{H} 2 \mathrm{~A}$ modification by Mec1 could be improved by increasing the concentration of Ddc2. We integrated plasmid PML105.45, which contains an extra copy of Ddc2 under a GAL1 promoter, at leu2-3,112 in the tel1 $\triangle y k u 80 \Delta$ strain (26). Indeed, increased expression of Ddc2 in this Mec1-only strain increased the amount of $\gamma$-H2AX (Figure 7A). In this instance, the total amount of $\gamma$-H2AX by Mec1 at $60 \mathrm{~min}$ in the Ddc2 overexpressed strains is nearly the same as that measured for the Tel1-only strain (Figure 7B). However, the distribution of $\gamma-\mathrm{H} 2 \mathrm{AX}$ sites is unaltered by the overexpression of Ddc2 since the MMD was unchanged from that seen in the absence of Ddc2 overexpression (Figure S2A). The 95\% CI for the MMD for the Ddc2 overexpression case is given by $\mathrm{MMD}_{\mathrm{Mec} 1, \mathrm{Ddc} 2} \mathrm{O} / \mathrm{E}=[12.5,14.1] \mathrm{kb}$ while the $\mathrm{MMD}$ for $\mathrm{Mec} 1$ is $\mathrm{MMD}_{\mathrm{Mec}}=[12.9,14.5] \mathrm{kb}$.

To determine the effects of Ddc2 overexpression and further test the 3D diffusion model of phosphorylation spreading by Mec1, we compared the theoretical model to the Ddc2overexpressed strain. Since Ddc2 forms an obligate heterodimer with Mec1 prior to its loading onto RPA-coated ssDNA $(23,24)$, the overexpression of Ddc2 should lead to an increased rate of Mec1 recruitment, $k_{\text {init }}$. Treating $k_{\text {init }}$ as a free parameter, while constraining all other model parameters to their previous values, we find that the $3 \mathrm{D}$ diffusion model agrees with the experimental data from the Mec1 strains in which Ddc2 is overexpressed (Figure 7C). The optimal $k_{\text {init }}$ is $0.25 /$ minute, 2.8 times the optimal $k_{\text {init }}$ for the Mec1-only strains where Ddc2 is expressed at wild-type levels.

\section{Discussion}

Although we know a great deal about the evolutionarily related phosphoinositol-3-kinase like kinases ATM and ATR, including their structure and phosphorylation targets (29), the manner by which these kinases reach their targets have not been well characterized. In this paper, we used Bayesian model selection to distinguish how Mec1 and Tell are able to create extended regions of $\gamma-\mathrm{H} 2 \mathrm{AX}$ after DNA damage. Our analysis shows that the 
experimental profile for $\mathrm{Mecl}$ is best matched by a 3D diffusive mechanism. However, we note that the 1D models also produce good fits to the Mec1 $\gamma-\mathrm{H} 2 \mathrm{AX}$ profile around the DSB (Figures S6-S8). Therefore, we do not rule out the possibility that Mec1 undergoes some $1 \mathrm{D}$ diffusion along the chromatin in addition to 3D diffusion. Meanwhile, the narrow shape of Tel1's $\gamma$-H2AX profile around the DSB is best fit by a directed sliding model and poorly fit by the 3D-spreading models (Figures 5B, S9-S14).

The $\gamma-\mathrm{H} 2 \mathrm{AX}$ profiles also provide us with insights into the recruitment process of the kinases. Since Tell is recruited to the nearly blunt ends created by HO endonuclease (13), whereas Mec1 requires that there be some 5' to 3' resection of the end (30), Tell would be expected to be activated before Mec1 and indeed this appears to be the case (Figures 1D$1 F)$. However, when we increased the expression of Ddc2, the kinetics of Tel1 and Mec1 activation were nearly identical, as the total $\gamma$-H2AX levels are now comparable by $60 \mathrm{~min}$ (Figure 7B). As Ddc2 is necessary for Mec1 loading onto RPA near the DSB, this result suggests that it is Mec1 binding to RPA rather than a delay in resection that limits the initial response.

One might also expect that, due to Tell's association with the MRX complex, Tell levels should increase in the absence of resection since MRX is recruited to nearly blunt DNA ends, but this was not found to be the case (Figures $1 \mathrm{~B}$ and 1E). We found that the amount of $\gamma-\mathrm{H} 2 \mathrm{AX}$ formation was somewhat reduced in G1-arrested nej $1 \Delta$ cells (where resection is impaired) compared to those in $y k u 80 \Delta$ cells (Figure S2B). It should be noted that there must be a number of MRX complexes recruited to the DSB in order that GFP-tagged proteins can be seen as a robust focus (31). It is possible that MRX-Tell binding is reduced by competitive binding of yKu70-yKu80, which also preferentially binds to dsDNA ends (32).

Previous studies have performed ChIP measurements of Mec1-Ddc2 and Tel1 adjacent to the DSB, finding that they increase roughly linearly over a time span longer than our experiments $(33,34)$. While these results could indicate an increasing number of kinase copies at the break site, our Bayes factor calculations suggest that that even with increasing kinase occupancy, the total kinase activity saturates - i.e. the rate of phosphorylation is constant over the 75 min of our experiment, not increasing. This was determined by considering both a constant phosphorylation rate and an increasing phosphorylation rate for all spreading models; we found that the $\gamma-\mathrm{H} 2 \mathrm{AX}$ profiles were best fit when the rate of phosphorylation remained constant over time (see Table S1 and Supplementary Information, Model Derivations). The constant rate is assumed for all models in the main text.

The initial recruitment of Mec1 to ssDNA only confines it to sites within a few kb of the $\mathrm{DSB}$ end and does not account for the rapid spreading of $\gamma-\mathrm{H} 2 \mathrm{AX}$ down the chromosome, since the rate of resection of DSB ends is only $4 \mathrm{~kb} / \mathrm{hr}(21)$. We note that while $\gamma-\mathrm{H} 2 \mathrm{AX}$ spreads rapidly over $50 \mathrm{~kb}$ from the break within an hour, $\mathrm{H} 2 \mathrm{~A}$ modifications continue to spread much more slowly over at least another $50 \mathrm{~kb}$, so that modifications around CEN3 - $100 \mathrm{~kb}$ from the DSB at MAT - are seen only after 8-10 h (35). This slower rate of modification parallels the rate of 5' to 3' resection and proves to be performed only by Mec1 (36). This activity suggests that, as new ssDNA is generated by the inexorable action 
of 5' to 3' exonucleases, Mec1 might re-load at newly synthesized ssDNA/RPA and proceed to diffuse from the new ssDNA to extend $\gamma-\mathrm{H} 2 \mathrm{AX}$ another $50 \mathrm{~kb}$ from the new site of entry.

Ddc2 assembles at the DSB in a Mec1-dependent manner $(26,27)$. Structural work on the Mec1-Ddc2 heterodimer suggests that it forms a stable focus at RPA-coated ssDNA with the coiled-coil domain of Ddc2 acting as a flexible linker so that Mec1 can freely phosphorylate nearby targets (28). If Mec1-Ddc2 remains at the break, then the looping mechanism would be the only possible option among the models that we considered, since the other mechanisms would require the kinase to detach from the DSB after recruitment. However, our analysis shows the predicted $\gamma$-H2AX profile from a looping mechanism does not fit the experimentally measured profile (Figures S3 and S4). In formulating the 3D mechanisms, we took into account the Kuhn length of the chromatin, as determined by both $\mathrm{HiC}$ and florescence microcopy experiments, and thus restricted the Kuhn length to 8.4-15 kb, the 95\% CI for the Kuhn length reported by Arbona et al. (37). Under these constraints, 3D diffusion is the preferred Mec1 mechanism, while looping interactions are unfavorable. Moreover, a 3D mechanism is supported by $\gamma-\mathrm{H} 2 \mathrm{AX}$ measurements around $\mathrm{RE}$, accounting for Mec1's ability to act in trans by phosphorylating histones on undamaged chromatin brought into close proximity with the DSB (Figures 3A and 6A). Additionally, Mec1 has been shown to phosphorylate histones clustered at pericentromeric regions when a DSB is generated close to one centromere (22).

Our conclusion of Tel1 sliding is seemingly at odds with prior results that support the idea that Tel1 is tethered to the break by the MRX complex, just as mammalian ATM is associated with MRN (38). ChIP measurements show that multiple copies of MRX (and presumably Tel1) remain associated with the DSB for 2-3 h (31), much longer than the 75 min duration of our experiments. However, our analysis has shown that looping, which we associate with a DSB bound kinase, is highly unlikely to be the mechanism employed by Tel1 since the theoretical predictions do not match the experimentally measured distribution and Bayesian analysis shows that 3D mechanisms are astronomically less probable than 1D sliding (Tables 1 and S1). Thus, although Tel1 is recruited to the DSB end, its subsequent action may not depend on the MRX complex.

The same mathematical formulation used to encode the directed sliding model can also be used to describe two other 1D spreading mechanisms: recruitment/assembly and loop extrusion. During recruitment/assembly, the arrival of a protein to the break facilitates the recruitment of subsequent proteins until a string of proteins is formed spanning many nucleosomes. In this scenario, the rate of recruitment of the next protein copy is analogous to $k_{\text {slide. }}$ This recruitment/assembly process has been suggested to occur in mammalian cells where the propagation of ATM down the chromosome is in part facilitated by the $\gamma-\mathrm{H} 2 \mathrm{AX}$ binding proteins MDC1 and possibly 53BP1 (39-41). MDC1 is capable of recruiting the MRN complex, which in turn recruits ATM further from the break, which in turn forms $\gamma$ $\mathrm{H} 2 \mathrm{AX}$ which act as MDC1 binding sites. The sequential assembly of MDC1 and ATM results in the propagation of $\gamma-\mathrm{H} 2 \mathrm{AX}$ away from the break $(33,35)$. While yeast lacks a MDC1 homolog, it is possible that its 53BP1 homolog, Rad9, may play a similar role in Tel1 mediated $\gamma-\mathrm{H} 2 \mathrm{AX}$ spreading (43). 
Results similar to 1D sliding would also be predicted if chromatin near a DSB were actively extruded into a loop, a process different from the looping model in which the chromosome only undergoes passive looping due to thermal fluctuations. In vitro experiments have shown that chromatin can be actively pulled through the ring-shaped cohesin or condensin complexes, resulting in extruded chromatin loops that grow over time $(29,30)$. Indeed, in yeast, cohesin and another SMC complex, Smc5,6 are recruited to sites of DSB damage (46-48). An analogous role for condensin has not been examined. We imagine a mechanism in which Tell remains tethered to the break while cohesin (or another SMC complex) sits near the break site and pulls in nearby chromatin through the ring. The histones that are pulled through the ring would therefore slide past Tell and be phosphorylated. Hence, the rate of loop extrusion is analogous to the rate $k_{\text {slide }}$ in the sliding model. From Bayesian parameter estimation for the sliding model for Tel1, we observe that the $95 \%$ CI for $k_{\text {slide }}$ overlaps with the estimated range for the speed of loop extrusion for cohesin (44). Also, since the rate of loop extrusion is a property of cohesin, we expect that $k_{\text {slide }}$ would be unaffected by the density of phosphorylatable sites in $\mathrm{H} 2 \mathrm{~A}$. Consistent with this notion, we found that $k_{\text {slide }}$ is unchanged in the strain where only $50 \%$ of the $\mathrm{H} 2 \mathrm{As}$ can be modified. Furthermore, unlike the sliding mechanism, loop extrusion has the added benefit of tethering Tell at the break end resulting in a stable focus. On the other hand, it should be noted that our measurements were made with G1-arrested cells, where cohesin or other SMC complexes may not be able to assemble (49-51). We further note that the profile of $\gamma$-H2AX spreading we have observed here is not apparently different from our previous observations when a DSB was induced in logarithmically growing cells $(12,22)$.

To our knowledge these are the first detailed comparisons of the mechanisms of spreading of ATM and ATR phosphorylation of histone H2A. They reveal that these two proteins, despite their evolutionary relationship, have adopted different strategies to propagate a signal away from a broken chromosome end.

\section{Methods and Materials}

\section{Strain Construction}

Standard yeast genome manipulation techniques were used to construct all strains and linear DNA and plasmids were introduced using the standard lithium acetate transformation protocol $(50,51)$. All strains used were variants of the strain JKM139 (54). The yeast strains used in this study can be found in Table S2. All primer sequences and plasmids used during strain construction are listed in Tables S3 and S4.

nej $1 \Delta:: \mathrm{HPH}$ mutants were constructed by amplifying pAG32 (HPH) using primers Nej1MXp1 and Nej1-MXp2 and $y k u 80 \Delta:: H P H$ mutants were constructed by PCR-amplifying pJH1515 using primers ku80MX18 and ku80MX19 to create a fragment containing hygromycin-resistance with homology to $N E J 1$ and $Y K U 80$, respectively. Linear DNA was introduced to the appropriate strain using lithium acetate transformation. htal-S129A mutants were generated by transforming strains with pBL13, which contains Cas 9 and a gRNA targeting HTA1, and the repair template BL327. Similarly, hta2-S129A mutants were generated by transforming strains with pBL14, which contains Cas 9 and a gRNA targeting 
HTA2, and the repair template BL331. The exact primer sequences of the repair oligos are shown in Table S3. S129A mutations were confirmed through sequencing by GENEWIZ.

Ddc2 was overexpressed in strain yKL019 by integrating PML105.45 (obtained from Maria Pia Longhese) which carries a copy of GAL1-Ddc2. PML105.45 was cut with ClaI and integrated at leu2-3,112 by transformation.

\section{Growth conditions and DSB induction}

Cells from a single colony were grown overnight in $5 \mathrm{ml}$ of YEPD, washed three times with YEP + 3\% lactic acid (YEP-Lac) and then grown in $350 \mathrm{ml}$ of YEP-Lac until log phase growth with a cell concentration between $5 \times 10^{6}$ cells $/ \mathrm{ml}$ and $8 \times 10^{6}$ cells $/ \mathrm{ml}$. $\alpha$ factor (United Biochemical) was added to the culture to a concentration of $\sim 5 \mu \mathrm{M}$ and maintained in the cell culture for at least for two doubling times before cell collection. G1 arrest was confirmed microscopically. After G1 arrest, $20 \%$ galactose was added to the YEP-Lac culture to a final concentration of $2 \%$ to induce $G A L:: H O$ expression, resulting in cutting at the MATa locus.

\section{Chromatin immunoprecipitation}

Cells were harvested from log-phase population before DSB induction and at various times after DSB induction. ChIP was carried out according to the protocol of Shroff et al. (9). Briefly, $45 \mathrm{ml}$ of culture were fixed and crosslinked with $1 \%$ formaldehyde for $10 \mathrm{~min}$, after which $2.5 \mathrm{ml}$ of $2.5 \mathrm{M}$ glycine was added for $5 \mathrm{~min}$ to quench the reaction. Cells were pelleted and washed 3 times with $4^{\circ} \mathrm{C}$ TBS. Yeast cell walls were disrupted by beating the cells with $425-600 \mu \mathrm{m}$ glass beads for $1 \mathrm{~h}$ in lysis buffer at $4^{\circ} \mathrm{C}$. The lysate was sonicated for $2 \mathrm{~min}$ to obtain chromatin fragments of $\sim 500 \mathrm{bp}$ in length. Debris was then pelleted and discarded, and equal volume of lysate was immunoprecipitated using $\gamma-\mathrm{H} 2 \mathrm{AX}$ antibody (abcam ab15083) for $1 \mathrm{~h}$ at $4^{\circ} \mathrm{C}$, followed by addition of Protein-A agarose beads (SigmaAldrich \#1719408001) for $1 \mathrm{~h}$ at $4^{\circ} \mathrm{C}$. The immunoprecipitate was then washed twice in $140 \mathrm{mM} \mathrm{NaCl}$ lysis buffer, once with $0.5 \mathrm{M} \mathrm{NaCl}$ lysis buffer, once with $0.25 \mathrm{M} \mathrm{LiCl}$ wash buffer and once with TE. Crosslinking was reversed at $65^{\circ} \mathrm{C}$ overnight followed by proteinase-K and glycogen addition for $2 \mathrm{~h}$. Protein and nucleic acids were separated by phenol extraction. $\mathrm{LiCl}$ was added to a final concentration of $400 \mathrm{mM} \mathrm{LiCl}$. DNA was precipitated using $99.5 \% \mathrm{EtOH}$. A second precipitation step was carried out using 75\% $\mathrm{EtOH}$ and the DNA resuspended in TE.

\section{Quantitative PCR}

$\gamma-\mathrm{H} 2 \mathrm{AX}$ levels around the DSB were assessed by quantitative PCR (qPCR) using the primer sequences listed in Table S5. qPCR of immunoprecipitated samples was carried out using a Rotor-Gene SYBR Green PCR Kit (Qiagen 204076) in a Qiagen Rotor-Gene Q real-time PCR machine. $\gamma-\mathrm{H} 2 \mathrm{AX}$ levels at all distances around the break were normalized to input as measured at the PHO5 locus with primers MT101 and MT102. 


\section{Computational Methods}

The predicted $\gamma$-H2AX profiles were simulated using the Gillespie algorithm (for the 1D diffusion model) or derived using master equations (for all other models). The models are described below and are derived in the Supplementary Information. There are 3 parameters specific to each model, plus an additional 3 parameters shared by all models. The shared parameters model the ChIP process.

All models begin with the formation of the DSB. Although induction of HO endonuclease is reasonably synchronous, the DSB does not form simultaneously in all cells, and we incorporate this variability into all models. As shown in Figure S1, the earliest cells incur a DSB was $\sim 12$ minutes after galactose exposure, presumably the time required for the transcription and translation of $\mathrm{HO}$ endonuclease. Over time, the DSB formed in more cells and by 30 minutes, $\sim 80 \%$ cells exhibited a DSB. The percent of cells without a break follows an exponential decay (Figure S1). After DSB formation, all models include the arrival of kinases (Mec1 or Tel1) to the break site, occurring at a constant rate $k_{\text {init }}$. For the 1D models, the parameter $k_{\text {init }}$ comprises both the recruitment and detachment from the DSB to begin traversing the chromosome.

The 3D diffusion, 1D diffusion and directed sliding models require that the kinase becomes activated upon arrival to the break site. Without this activation, these models would not predict preferential phosphorylation of $\mathrm{H} 2 \mathrm{As}$ close to the break site (see the Supplementary Information for exceptions to this requirement and a further discussion of activation). A precedent for such activation can be found in an in vivo study of ATM, the mammalian homolog of Tel1, in which ATM autophosphorylation and activation occurs through its association with the MRN complex (55). In budding yeast, Mec1 phosphorylates sites on its partner, Ddc2, but only does so after DSB formation, presumably when it is bound to the break region (14). For simplicity, the initial recruitment rate parameter $k_{\text {init }}$ also encompasses the activation step.

\section{Looping Model}

We model the chromosome as a worm-like chain at thermodynamic equilibrium, which has previously been shown to predict the frequencies of physical contact between chromosomal loci in yeast (56) (and similar polymer models have accurately predicted contact frequencies as well as the $3 \mathrm{D}$ positioning of genes in the yeast nucleus $(24,37$, 57)). The thermodynamic equilibrium assumption is justified because we estimate that loci $50 \mathrm{~kb}$ away from each other should encounter each other every few minutes (every few seconds for loci $10 \mathrm{~kb}$ away from each other), which is much faster than the 30-60 minute time scale over which H2A phosphorylation occurs (see Supplementary Information, Looping Model Derivation for a description of this estimate).

The probability $P_{\text {loop }}$ that the chromosome is in a looped state - i.e. the probability that the DSB-bound kinase is in contact with an H2A located $x$ kilobases away - depends on the 
ratio of $x$ to $l$, where $l$ is the chromatin's Kuhn length. For H2As very close to the break ( $x$ $<<l$ ), the stiffness of the chromosome makes the formation of a loop highly improbable since the energy required to bend the chromatin into a small loop is prohibitive. For H2As far from the break site $(x>>l)$, loops are unlikely to form since the kinase and target histones tend to occupy different regions of space. Loop formation is most probable in the regime of $x \approx l$. Specifically, the $P_{\text {loop }}(x / l)$ is maximal at $x=1.7 l(58)$. When the chromosome is in the looped state, the kinase phosphorylates the $\mathrm{H} 2 \mathrm{~A}$ at a rate $k_{c a t}$. We introduce the model parameter $\varphi$, which is the product of $k_{c a t}$ and factors that take into account how close to the $\mathrm{H} 2 \mathrm{~A}$ and in what orientations the kinase can be in order to phosphorylate the $\mathrm{H} 2 \mathrm{~A}$. The looping model therefore has three parameters: $k_{\text {init }}, l$, and $\varphi$

\section{$3 D$ diffusion model}

Following the arrival and activation of a kinase at the DSB, the kinase leaves the break site and then diffuses along some 3D trajectory, hitting H2As and phosphorylating them. An $\mathrm{H} 2 \mathrm{~A}$ near the break is likely to be contacted by the kinase because many of the possible trajectories go through this $\mathrm{H} 2 \mathrm{~A}$. An $\mathrm{H} 2 \mathrm{~A}$ far from the break is less likely to be contacted because few trajectories go through this $\mathrm{H} 2 \mathrm{~A}$ - most trajectories go off to other parts of the nucleus far from this $\mathrm{H} 2 \mathrm{~A}$. While this is clearly true for short trajectories that cross the nucleus only once, can this still apply to long trajectories? When the kinase continues diffusing, it presumably crosses the nucleus a large number of times over the course of the hour-long experiment, so in the later stages of its diffusion, the kinase is equally likely to hit any $\mathrm{H} 2 \mathrm{~A}$ in the nucleus. However, as there are so many H2As in the nucleus, it is reasonable to assume that the catalytic rate is only fast enough for the kinase to phosphorylate a small fraction of the H2As in the nucleus. Therefore, the later stages of a kinase's diffusion might add only a negligible $\gamma-\mathrm{H} 2 \mathrm{AX}$ signal. The predominant $\gamma-\mathrm{H} 2 \mathrm{AX}$ signal comes from the early stage of diffusion, which results in the preferential phosphorylation of H2As near the break.

To predict the probability that the kinase will hit an $\mathrm{H} 2 \mathrm{~A}$ during the early stage of diffusion, we use the simple approximation $P_{\text {contact }}=a / R(59)$, where $a$ is the radius of the H2A and $R$ is the $3 \mathrm{D}$ distance between the $\mathrm{H} 2 \mathrm{~A}$ and the kinase at the beginning of the kinase's trajectory ( $R$ is taken to be the root mean squared distance from the H2A to the DSB, given by a worm-like chain treatment of the chromatin at thermodynamic equilibrium). We assume many copies of the kinase come on and off the DSB, where $k_{\text {leaveDSB }}$ is the rate at which a kinase leaves the DSB: if kinases come off the DSB at a higher frequency, more kinases will be diffusing and contacting H2As, so H2A phosphorylation will occur at a faster rate. The $3 \mathrm{D}$ diffusion model has three parameters: $k_{\text {init }}, l$, and $\omega$, where $\omega$ is a product of $k_{\text {leaveDSB }}, a$ and other factors that describe how likely the kinase is to contact an H2A (see Supplementary Information for details).

\section{$1 D$ diffusion model}


In the 1D diffusion model, the chromatin fiber is treated as a 1D filament of H2As. Starting from the break site, a kinase moves along the chromatin by sliding between adjacent $\mathrm{H} 2 \mathrm{~A}$ histones. At each step, the kinase phosphorylates $\mathrm{H} 2 \mathrm{~A}$ at a rate $k_{\text {cat }}$. The kinase is allowed to move in the direction away from the break site or towards the break site with equal probability. The overall speed of the kinase's motion is governed by the $1 \mathrm{D}$ diffusion coefficient $D$. For simplicity, we assume that the kinase does not permanently detach from the chromosome - i.e. we assume the time scale of falling off is greater than the duration of the experiment. Over a fixed time interval, the kinase is more likely to phosphorylate $\mathrm{H} 2 \mathrm{As}$ that are closer to the DSB than those that are further because it takes a long time for a random walk to reach a distant location. The simulations include many copies of the kinase diffusing concurrently on the chromosome (this is further discussed in the Supplementary Information). The parameters of the $1 \mathrm{D}$ diffusion model are $k_{\text {init }}, D$, and $k_{\text {cat }}$.

\section{Directed sliding model}

As in the 1D diffusion model, the chromatin is treated as a 1D filament of H2A histones. Starting from the break site, the kinase slides along the chromatin at a rate $k_{\text {slide }}$ until it falls off the chromosome at a rate $k_{\text {off }}$. We assume the kinase phosphorylates all H2As along the way between the break site and wherever the kinase happens to fall off the chromosome, and the kinase cannot rebind to $\mathrm{H} 2 \mathrm{As}$ that it has already phosphorylated (i.e. the kinase cannot backtrack); therefore, the kinase slides unidirectionally away from the break site. In this model, H2As close to the break are more likely to be phosphorylated because a kinase is unlikely to reach distant $\mathrm{H} 2 \mathrm{As}$ before it falls off the chromatin. The parameters of the directed sliding model are $k_{\text {init }}, k_{\text {slide }}$, and $k_{\text {off }}$.

\section{Acknowledgments}

We thank members of the Haber and Kondev groups and Dr. Douglas Theobald for helpful discussions. We also thank Dr. Maria Pia Longhese for providing us with plasmid PML105.45. Funding for JEH is provided by GM20056 and R35127029. Funding for JK is provided by the National Science Foundation grants DMR-1610737 and MRSEC1420382, and by the Simons Foundation. 


\section{References}

1. Dekker J, Misteli T. Long-Range Chromatin Interactions. Cold Spring Harb Perspect Biol. 2015 Oct;7(10):a019356.

2. Schoenfelder S, Fraser P. Long-range enhancer-promoter contacts in gene expression control. Nat Rev Genet. 2019 Aug;20(8):437-55.

3. Jasin M, Rothstein R. Repair of strand breaks by homologous recombination. Cold Spring Harb Perspect Biol. 2013 Nov 1;5(11):a012740.

4. Zhang Y, Dudko OK. First-Passage Processes in the Genome. Annu Rev Biophys. 2016 Jul 5;45(1):117-34.

5. Talbert PB, Henikoff S. Spreading of silent chromatin: inaction at a distance. Nat Rev Genet. 2006 Oct;7(10):793-803.

6. Downs JA, Allard S, Jobin-Robitaille O, Javaheri A, Auger A, Bouchard N, et al. Binding of Chromatin-Modifying Activities to Phosphorylated Histone H2A at DNA Damage Sites. Mol Cell. 2004 Dec;16(6):979-90.

7. Redon C, Pilch DR, Rogakou EP, Orr AH, Lowndes NF, Bonner WM. Yeast histone 2A serine 129 is essential for the efficient repair of checkpoint-blind DNA damage. EMBO Rep. 2003 Jul;4(7):678-84.

8. Rogakou EP, Boon C, Redon C, Bonner WM. Megabase Chromatin Domains Involved in DNA Double-Strand Breaks in Vivo. J Cell Biol. 1999 Sep 6;146(5):905-16.

9. Shroff R, Arbel-Eden A, Pilch D, Ira G, Bonner WM, Petrini JH, et al. Distribution and Dynamics of Chromatin Modification Induced by a Defined DNA DoubleStrand Break. Curr Biol. 2004 Oct;14(19):1703-11.

10. Natale F, Rapp A, Yu W, Maiser A, Harz H, Scholl A, et al. Identification of the elementary structural units of the DNA damage response. Nat Commun. 2017 Jun $12 ; 8: 15760$.

11. Blackford AN, Jackson SP. ATM, ATR, and DNA-PK: The Trinity at the Heart of the DNA Damage Response. Mol Cell. 2017 Jun;66(6):801-17.

12. Corcoles-Saez I, Dong K, Cha RS. Versatility of the Mec1ATM/ATR signaling network in mediating resistance to replication, genotoxic, and proteotoxic stresses. Curr Genet. 2019 Jun;65(3):657-61.

13. Gobbini E, Cassani C, Villa M, Bonetti D, Longhese M. Functions and regulation of the MRX complex at DNA double-strand breaks. Microb Cell. 2016 Aug $1 ; 3(8): 329-37$. 
14. Paciotti V, Clerici M, Lucchini G, Longhese MP. The checkpoint protein Ddc2, functionally related to S. pombe Rad26, interacts with Mec1 and is regulated by Mec1-dependent phosphorylation in budding yeast. Genes Dev. 2000 Aug 15;14(16):2046-59.

15. Bandhu A, Kang J, Fukunaga K, Goto G, Sugimoto K. Ddc2 Mediates Mec1 Activation through a Ddc1- or Dpb11-Independent Mechanism. Toczyski DP, editor. PLoS Genet. 2014 Feb 20;10(2):e1004136.

16. Moore JK, Haber JE. Cell cycle and genetic requirements of two pathways of nonhomologous end-joining repair of double-strand breaks in Saccharomyces cerevisiae. Mol Cell Biol. 1996 May;16(5):2164-73.

17. Emerson $\mathrm{CH}$, Bertuch AA. Consider the workhorse: Nonhomologous end-joining in budding yeast. Biochem Cell Biol. 2016 Oct;94(5):396-406.

18. Ira G, Pellicioli A, Balijja A, Wang X, Fiorani S, Carotenuto W, et al. DNA end resection, homologous recombination and DNA damage checkpoint activation require CDK1. Nature. 2004 Oct;431(7011):1011-7.

19. Aylon Y, Liefshitz B, Kupiec M. The CDK regulates repair of double-strand breaks by homologous recombination during the cell cycle. EMBO J. 2004 Dec 8;23(24):4868-75.

20. Shim EY, Chung W-H, Nicolette ML, Zhang Y, Davis M, Zhu Z, et al. Saccharomyces cerevisiae Mre11/Rad50/Xrs2 and Ku proteins regulate association of Exo1 and Dna2 with DNA breaks. EMBO J. 2010 Oct 6;29(19):3370-80.

21. Eapen VV, Sugawara N, Tsabar M, Wu W-H, Haber JE. The Saccharomyces cerevisiae Chromatin Remodeler Fun30 Regulates DNA End Resection and Checkpoint Deactivation. Mol Cell Biol. 2012 Nov 15;32(22):4727-40.

22. Lee C-S, Lee K, Legube G, Haber JE. Dynamics of yeast histone H2A and H2B phosphorylation in response to a double-strand break. Nat Struct Mol Biol. 2014 Jan;21(1):103-9.

23. Li J, Coïc E, Lee K, Lee C-S, Kim J-A, Wu Q, et al. Regulation of Budding Yeast Mating-Type Switching Donor Preference by the FHA Domain of Fkh1. Hunter N, editor. PLoS Genet. 2012 Apr 5;8(4):e1002630.

24. Avşaroğlu B, Bronk G, Li K, Haber JE, Kondev J. Chromosome-refolding model of mating-type switching in yeast. Proc Natl Acad Sci U S A. 2016 Nov 8;113(45):E6929-38.

25. Talbert PB, Henikoff S. Spreading of silent chromatin: inaction at a distance. Nat Rev Genet. 2006 Oct;7(10):793-803. 
26. Clerici M. Hyperactivation of the yeast DNA damage checkpoint by TEL1 and DDC2 overexpression. EMBO J. 2001 Nov 15;20(22):6485-98.

27. Memisoglu G, Lanz MC, Eapen VV, Jordan JM, Lee K, Smolka MB, et al. Mec1ATR Autophosphorylation and Ddc2ATRIP Phosphorylation Regulates DNA Damage Checkpoint Signaling. Cell Rep. 2019 Jul;28(4):1090-1102.e3.

28. Deshpande I, Seeber A, Shimada K, Keusch JJ, Gut H, Gasser SM. Structural Basis of Mec1-Ddc2-RPA Assembly and Activation on Single-Stranded DNA at Sites of Damage. Mol Cell. 2017 Oct;68(2):431-445.e5.

29. Waterman D, Haber JE, Smolka MB. Checkpoint responses to DNA double-strand breaks. Annu Rev Biochem. 2020;

30. Zou L, Elledge SJ. Sensing DNA damage through ATRIP recognition of RPAssDNA complexes. Science. 2003 Jun 6;300(5625):1542-8.

31. Lisby M, Barlow JH, Burgess RC, Rothstein R. Choreography of the DNA Damage Response: Spatiotemporal Relationships among Checkpoint and Repair Proteins. Cell. 2004 Sep 17;118(6):699-713.

32. Ristic D. Rad52 and Ku bind to different DNA structures produced early in doublestrand break repair. Nucleic Acids Res. 2003 Sep 15;31(18):5229-37.

33. Gobbini E, Villa M, Gnugnoli M, Menin L, Clerici M, Longhese MP. Sae2 Function at DNA Double-Strand Breaks Is Bypassed by Dampening Tel1 or Rad53 Activity. Jinks-Robertson S, editor. PLOS Genet. 2015 Nov 19;11(11):e1005685.

34. Clerici M, Trovesi C, Galbiati A, Lucchini G, Longhese MP. Mec1/ATR regulates the generation of single-stranded DNA that attenuates Tel1/ATM signaling at DNA ends. EMBO J. 2014 Feb 3;33(3):198-216.

35. Dotiwala F, Harrison JC, Jain S, Sugawara N, Haber JE. Mad2 Prolongs DNA Damage Checkpoint Arrest Caused by a Double-Strand Break via a CentromereDependent Mechanism. Curr Biol. 2010 Feb;20(4):328-32.

36. Kim J-A, Kruhlak M, Dotiwala F, Nussenzweig A, Haber JE. Heterochromatin is refractory to $\gamma-\mathrm{H} 2 \mathrm{AX}$ modification in yeast and mammals. J Cell Biol. $2007 \mathrm{Jul}$ $16 ; 178(2): 209-18$.

37. Arbona J-M, Herbert S, Fabre E, Zimmer C. Inferring the physical properties of yeast chromatin through Bayesian analysis of whole nucleus simulations. Genome Biol. 2017 Dec;18(1).

38. Lisby M, Barlow JH, Burgess RC, Rothstein R. Choreography of the DNA Damage Response. Cell. 2004 Sep;118(6):699-713. 
39. Scully R, Xie A. Double strand break repair functions of histone H2AX. Mutat Res Mol Mech Mutagen. 2013 Oct;750(1-2):5-14.

40. van Attikum H, Gasser SM. Crosstalk between histone modifications during the DNA damage response. Trends Cell Biol. 2009 May;19(5):207-17.

41. Coster G, Goldberg M. The cellular response to DNA damage: A focus on MDC1 and its interacting proteins. Nucleus. 2010 Mar 1;1(2):166-78.

42. Lou Z, Minter-Dykhouse K, Franco S, Gostissa M, Rivera MA, Celeste A, et al. MDC1 Maintains Genomic Stability by Participating in the Amplification of ATMDependent DNA Damage Signals. Mol Cell. 2006 Jan;21(2):187-200.

43. Ferrari M, Dibitetto D, De Gregorio G, Eapen VV, Rawal CC, Lazzaro F, et al. Functional Interplay between the 53BP1-Ortholog Rad9 and the Mre11 Complex Regulates Resection, End-Tethering and Repair of a Double-Strand Break. Nitiss JL, editor. PLoS Genet. 2015 Jan 8;11(1):e1004928.

44. Fudenberg G, Abdennur N, Imakaev M, Goloborodko A, Mirny LA. Emerging Evidence of Chromosome Folding by Loop Extrusion. Cold Spring Harb Symp Quant Biol. 2017 Jan 1;82:45-55.

45. Hassler M, Shaltiel IA, Kschonsak M, Simon B, Merkel F, Thärichen L, et al. Structural Basis of an Asymmetric Condensin ATPase Cycle. Mol Cell. 2019 Jun;74(6):1175-1188.e9.

46. Ström L, Lindroos HB, Shirahige K, Sjögren C. Postreplicative Recruitment of Cohesin to Double-Strand Breaks Is Required for DNA Repair. Mol Cell. 2004 Dec;16(6):1003-15.

47. Ünal E, Arbel-Eden A, Sattler U, Shroff R, Lichten M, Haber JE, et al. DNA Damage Response Pathway Uses Histone Modification to Assemble a Double-Strand BreakSpecific Cohesin Domain. Mol Cell. 2004 Dec;16(6):991-1002.

48. Ström L, Sjögren C. Chromosome segregation and double-strand break repair - a complex connection. Curr Opin Cell Biol. 2007 Jun;19(3):344-9.

49. Srinivasan M, Petela NJ, Scheinost JC, Collier J, Voulgaris M, B Roig M, et al. Scc2 counteracts a Wapl-independent mechanism that releases cohesin from chromosomes during G1. eLife. 2019 Jun 21;8.

50. Lazar-Stefanita L, Scolari VF, Mercy G, Muller H, Guérin TM, Thierry A, et al. Cohesins and condensins orchestrate the 4D dynamics of yeast chromosomes during the cell cycle. EMBO J. 2017 Sep 15;36(18):2684-97.

51. Schalbetter SA, Goloborodko A, Fudenberg G, Belton J-M, Miles C, Yu M, et al. SMC complexes differentially compact mitotic chromosomes according to genomic context. Nat Cell Biol. 2017 Sep;19(9):1071-80. 
52. Gietz RD, Woods RA. Transformation of yeast by lithium acetate/single-stranded carrier DNA/polyethylene glycol method. Methods Enzymol. 2002;350:87-96.

53. Wach A, Brachat A, Pöhlmann R, Philippsen P. New heterologous modules for classical or PCR-based gene disruptions in Saccharomyces cerevisiae. Yeast Chichester Engl. 1994 Dec;10(13):1793-808.

54. White CI, Haber JE. Intermediates of recombination during mating type switching in Saccharomyces cerevisiae. EMBO J. 1990 Mar;9(3):663-73.

55. Kozlov SV, Graham ME, Jakob B, Tobias F, Kijas AW, Tanuji M, et al. Autophosphorylation and ATM Activation ADDITIONAL SITES ADD TO THE COMPLEXITY. J Biol Chem. 2011 Mar 18;286(11):9107-19.

56. Dekker J. Mapping in Vivo Chromatin Interactions in Yeast Suggests an Extended Chromatin Fiber with Regional Variation in Compaction. J Biol Chem. 2008 Dec $12 ; 283(50): 34532-40$.

57. Avşaroğlu B, Bronk G, Gordon-Messer S, Ham J, Bressan DA, Haber JE, et al. Effect of Chromosome Tethering on Nuclear Organization in Yeast. Toda T, editor. PLoS ONE. 2014 Jul 14;9(7):e102474.

58. Dekker J, Rippe K, Dekker M, Kleckner N. Capturing Chromosome Conformation. Science. 2002 Feb 15;295(5558):1306-11.

59. Berg H. Random Walks in Biology. New, expanded. Princeton University Press; 1993. 


\section{Figures}
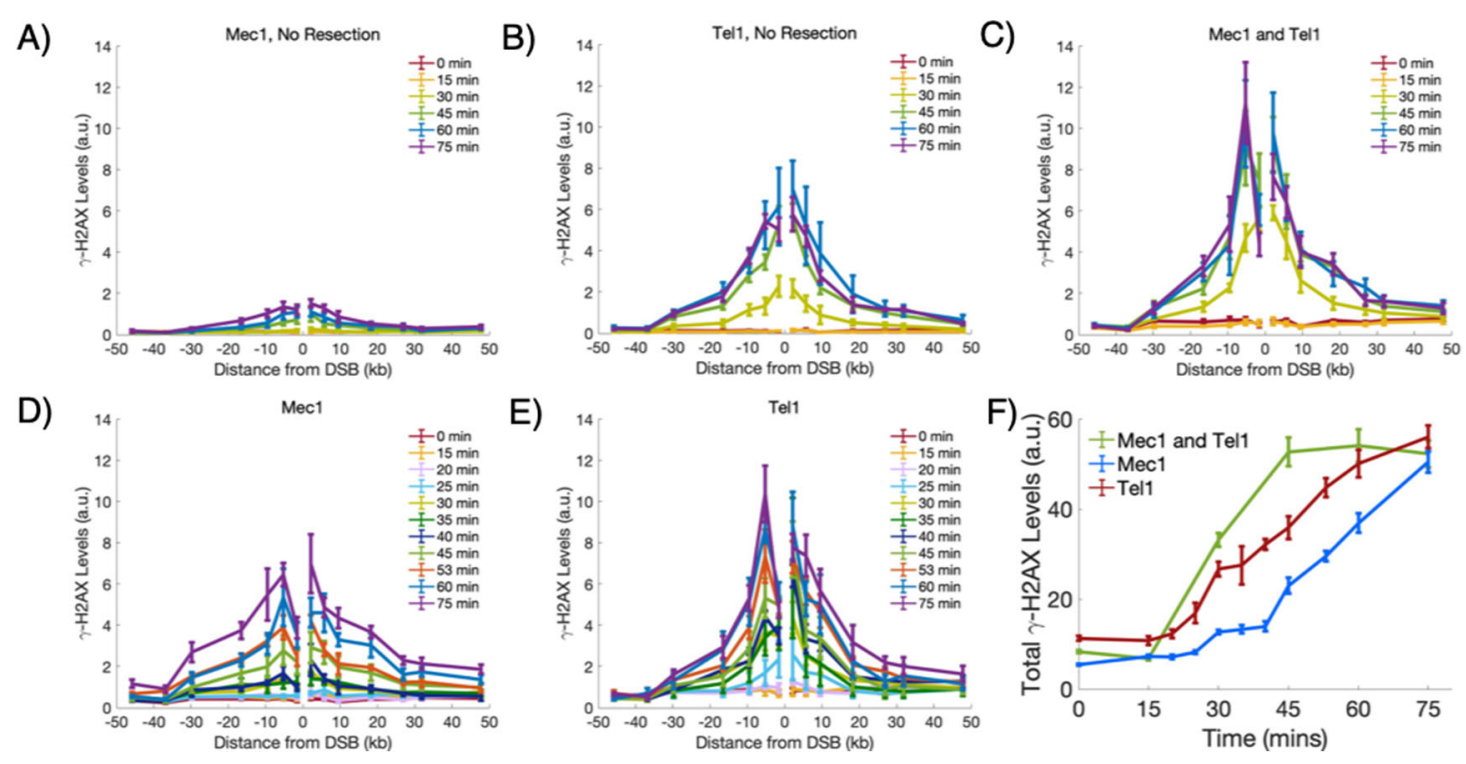

\section{Figure 1: Experimental $\gamma-\mathrm{H} 2 \mathrm{AX}$ profiles by Mec1 and Tel1}

$\gamma$-H2AX spreading as measured by ChIP-qPCR after generating a break at the MAT locus. ChIP was performed using an antibody specific to $\gamma-\mathrm{H} 2 \mathrm{AX}$. All cells were arrested in G1 with end-joining prevented by $n e j 1 \Delta$ or $y k u 80 \Delta$. In all plots, error bars represent standard error of the mean from $n \geq 3$ measurements.
A) $\gamma$-H2AX spreading by Mec1 in $n e j 1 \Delta$ cells.
B) $\gamma$-H2AX spreading by Mec1 in nej $1 \Delta$ cells.
C) $\gamma-\mathrm{H} 2 \mathrm{AX}$ spreading by both Mec1 and Tel1 in $k u 80 \Delta$ cells.
D) $\gamma$-H2AX spreading by Mec 1 in $k u 80 \Delta$ cells.

E) $\gamma$-H2AX spreading by Tell in $k u 80 \Delta$ cells.

F) Total $\gamma$-H2AX levels by both Mec1 and Tel1 (green), only Mec1 (blue) and only Tel1 (red) in $k u 80 \Delta$ cells. 

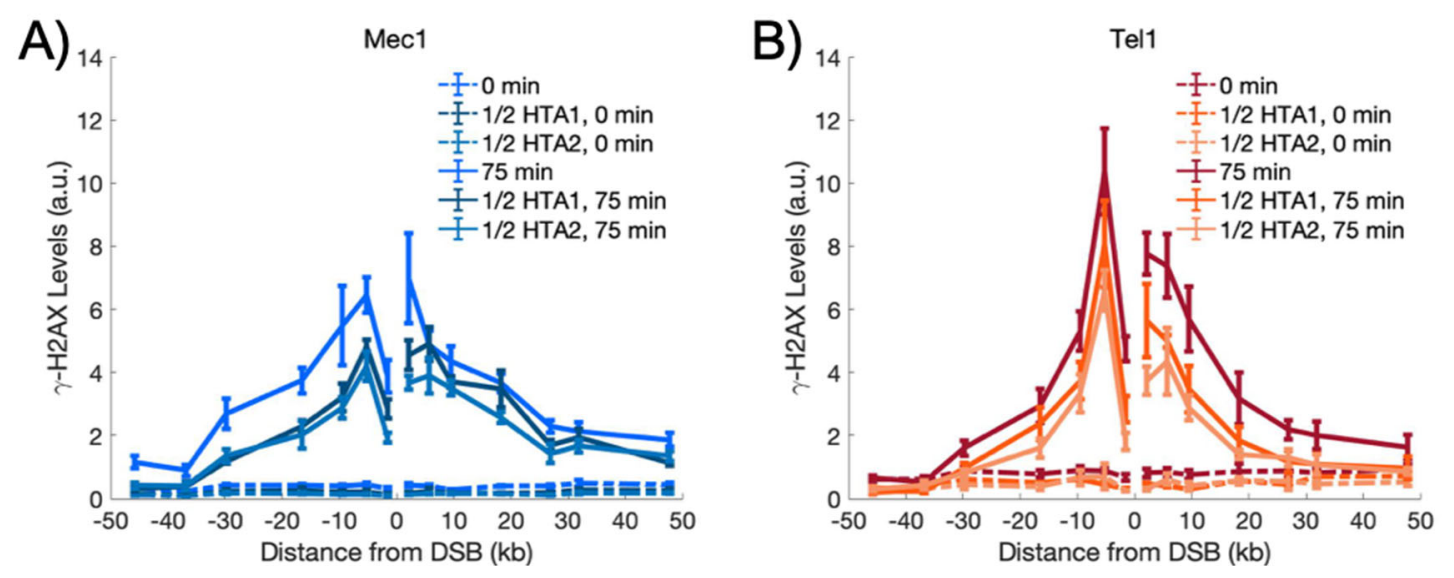

Figure 2: Experimental $\gamma-\mathrm{H} 2 \mathrm{AX}$ profiles by Mec1 and Tel1 when $50 \%$ of $\mathrm{H} 2 \mathrm{~A}$ sites cannot be phosphorylated.

Phosphorylation spreading in strains where one of the two HTA genes is mutated to HTA$S 129 A$, rendering half the H2A sites non-phosphorylatable. Experimentally measured $\gamma-$ $\mathrm{H} 2 \mathrm{AX}$ profiles in the presence $50 \%$ phosphorylatable H2A by Mec1 (A) or Tel1 (B) are shown for $0 \mathrm{~min}$ (dashed lines) and $75 \mathrm{~min}$ (solid lines). "1/2 HTAl" refers to the presence of an hta2-S129A mutation, while "1/2 HTA2" carries the hta1-S129A mutation. Error bars represent standard error of the mean from $n \geq 3$ measurements. 

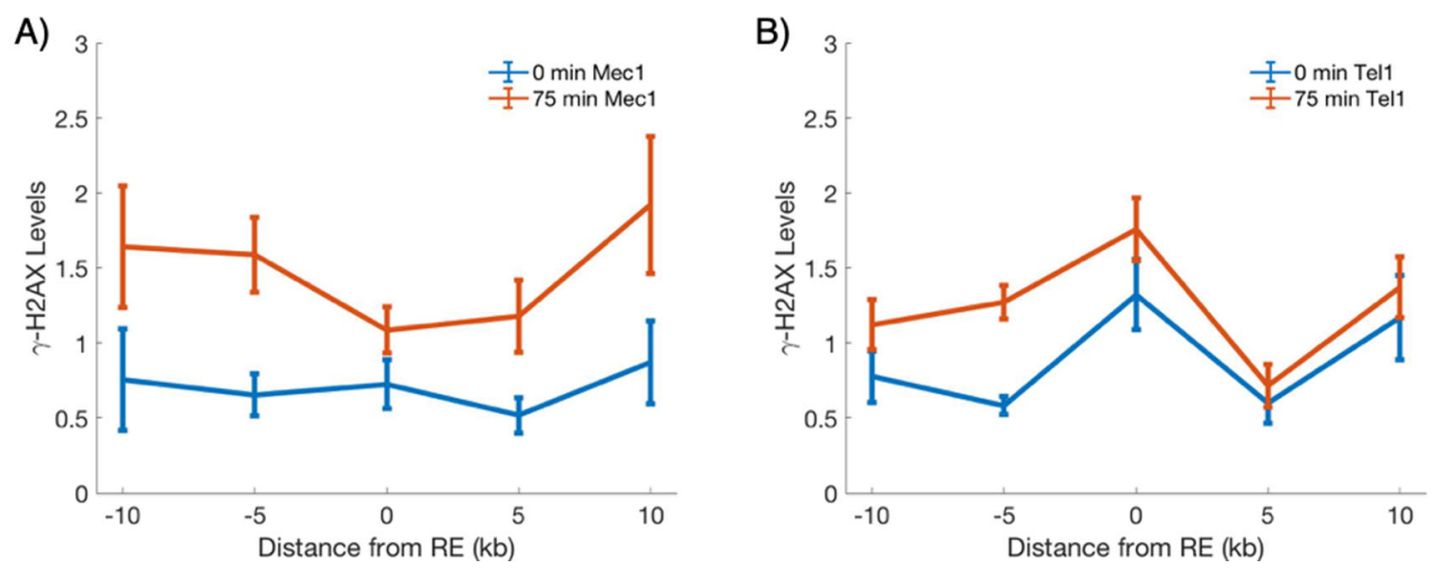

Figure 3: $\gamma$-H2AX measurements around RE

$\gamma$-H2AX levels measured around the recombination enhancer (RE), a locus on ChrIII known to interact with the MAT locus by forming a chromatin loop. A) $\gamma-\mathrm{H} 2 \mathrm{AX}$ formation around RE by Mec1. Error bars represent standard error of the mean from $n \geq 4$ measurements. B) $\gamma$-H2AX formation around RE by Tel1. Error bars represent standard error of the mean from $n \geq 4$ measurements. 

A) Looping
B) 3D Diffusion

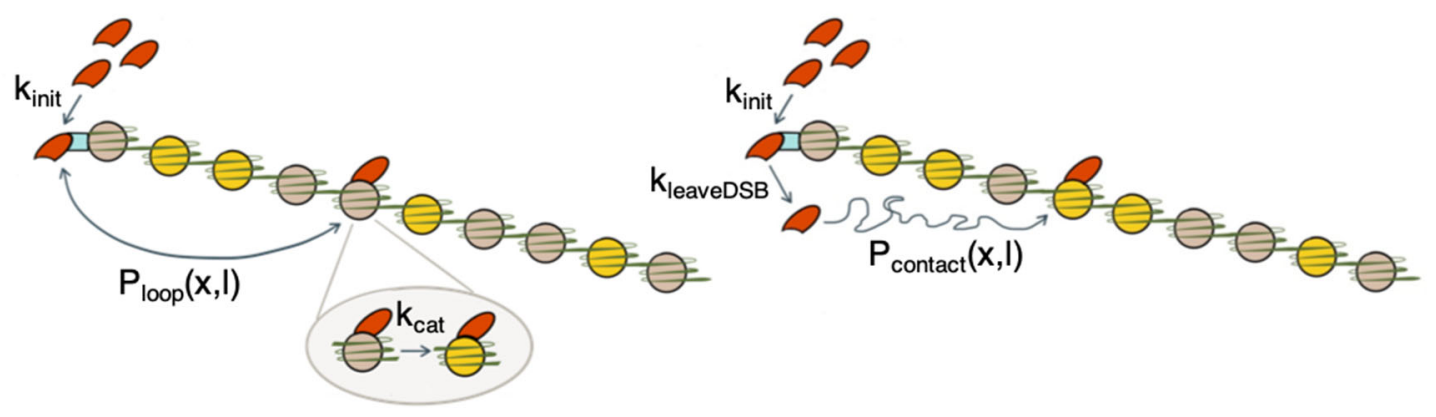

C) 1D Diffusion

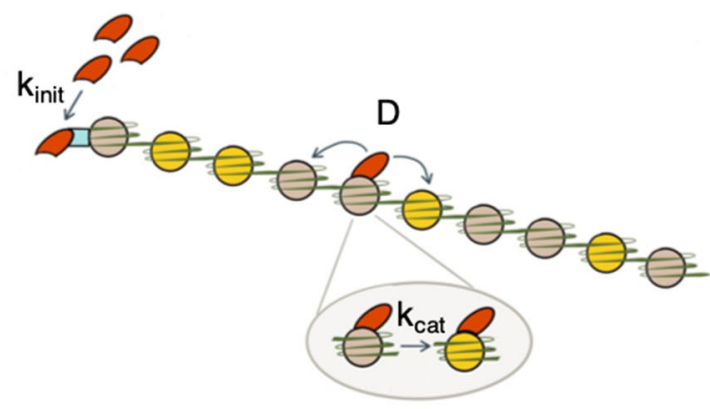

D) Directed Sliding

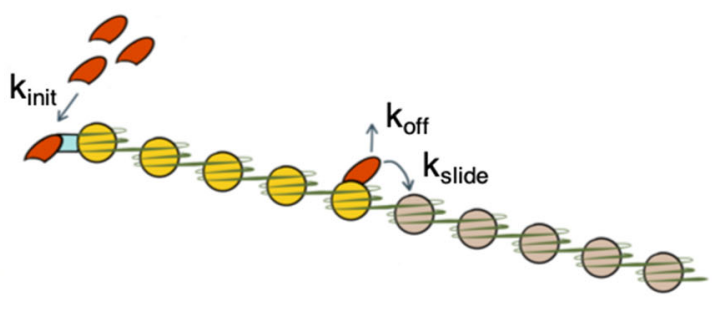

Kinase $\quad \square$ Binding Protein

$\mathrm{H} 2 \mathrm{~A}$

○े-H2AX

DNA

\section{E) Chromatin Fragment Recovery}
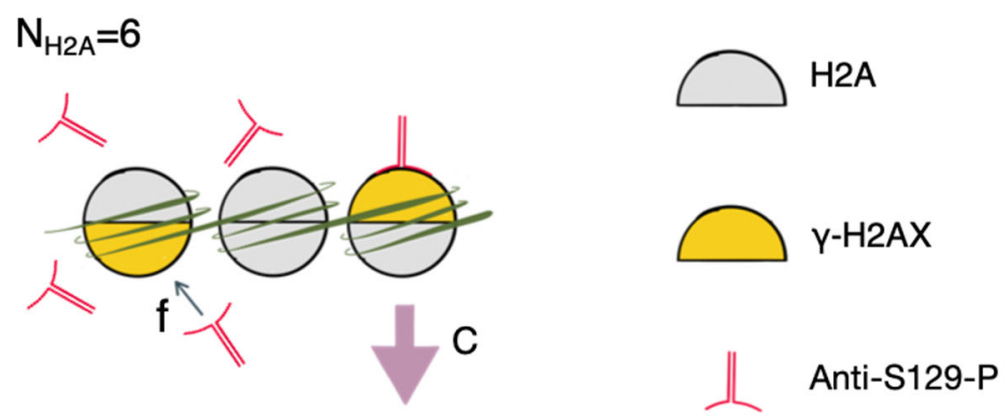


\section{Figure 4: Schematic of phosphorylation spreading mechanisms}

Schematics of looping (A), 3D diffusion (B), 1D diffusion (C) and directed sliding (D) mechanisms are depicted. The kinases Mec1 and Tell (red) are recruited to the DSB at a rate $k_{\text {init }}$ to RPA and MRX respectively (teal). For the one dimensional models, 1D diffusion and directed sliding, kinit comprises both the kinase's recruitment to and detachment from the DSB to begin translocating along the chromatin. The kinase proceeds to phosphorylate $\mathrm{H} 2 \mathrm{~A}$ (grey) to form $\gamma-\mathrm{H} 2 \mathrm{AX}$ (yellow) using one of the four mechanisms.

A) In the looping model, the kinase remains tethered at the DSB and forms a looped conformation with probability $P_{l o o p}(x, l)$, where $x$ describes the distance of the target H2A from the break and $l$ is the Kuhn length of the chromatin. $P_{l o o p}(x, l)$ is formulated using a worm-like chain polymer model. The kinase phosphorylates the $\mathrm{H} 2 \mathrm{~A}$ at a rate $k_{\text {cat }}$ after contact is established with the $\mathrm{H} 2 \mathrm{~A}$.

B) In the 3D diffusion model, the kinase transiently binds to the DSB where it is activated and released at a rate kleaveDSB. The activated kinase diffuses away from the DSB until it encounters and phosphorylates an H2A. $P_{\text {contact }}(x, l)$ is the probability that the kinase will come into contact with an H2A located a distance $\mathrm{x}$ away from the break.

C) In the 1D diffusion model, the kinase is recruited to the DSB and proceeds to diffuse along the chromatin with a one-dimensional diffusion coefficient $D$. The kinase phosphorylates the $\mathrm{H} 2 \mathrm{~A}$ that it comes into contact at a rate $k_{\text {cat }}$.

D) In the directed sliding model, the kinase moves unidirectionally away from the DSB along the chromatin. The kinase slides onto adjacent histones at a rate $k_{\text {slide }}$ and phosphorylates all $\mathrm{H} 2 \mathrm{As}$ encountered until the kinase detaches from the chromatin at a rate $k_{\text {off }}$.

E) Sonication during ChIP yields chromatin of $\sim 500 \mathrm{bp}$. The parameter $N_{H 2 A}$ describes the average number of $\mathrm{H} 2 \mathrm{As}$ (grey) present on the DNA fragment. In this depiction, $\mathrm{N}_{\mathrm{H} 2 \mathrm{~A}}=6$. Each $\gamma-\mathrm{H} 2 \mathrm{AX}$ (yellow) is bound by an antibody (red) with probability $f$. Antibody binding to one $\gamma-\mathrm{H} 2 \mathrm{AX}$ is independent of the other $\mathrm{H} 2 \mathrm{~A}$ sites on the same chromatin fragment. The binding of one antibody is sufficient for the pull down of the entire fragment (purple arrow). The recovery of the fragment is multiplied by the parameter $C$ to account for the loss of DNA during the wash steps in ChIP. 

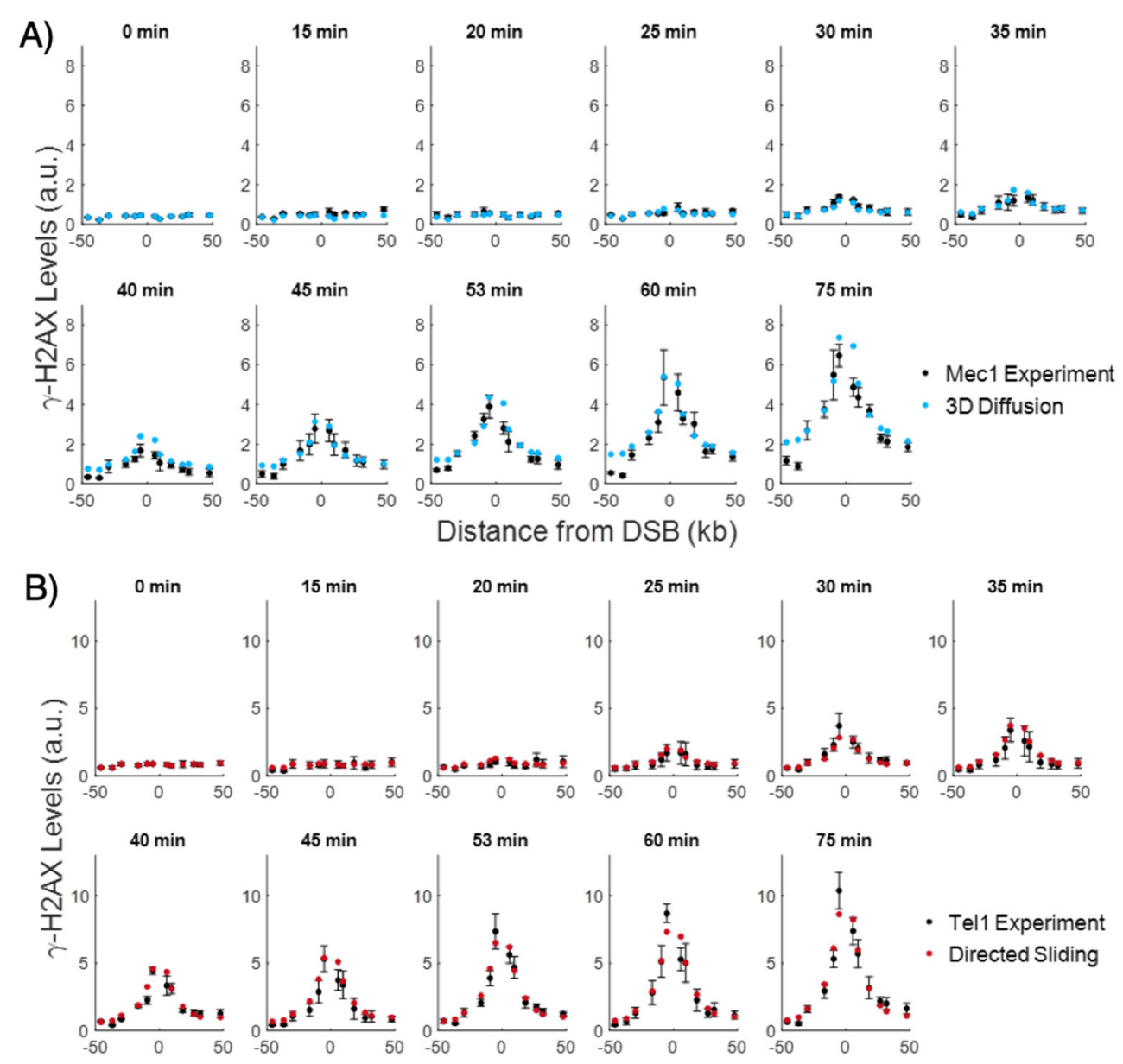

Distance from DSB $(\mathrm{kb})$
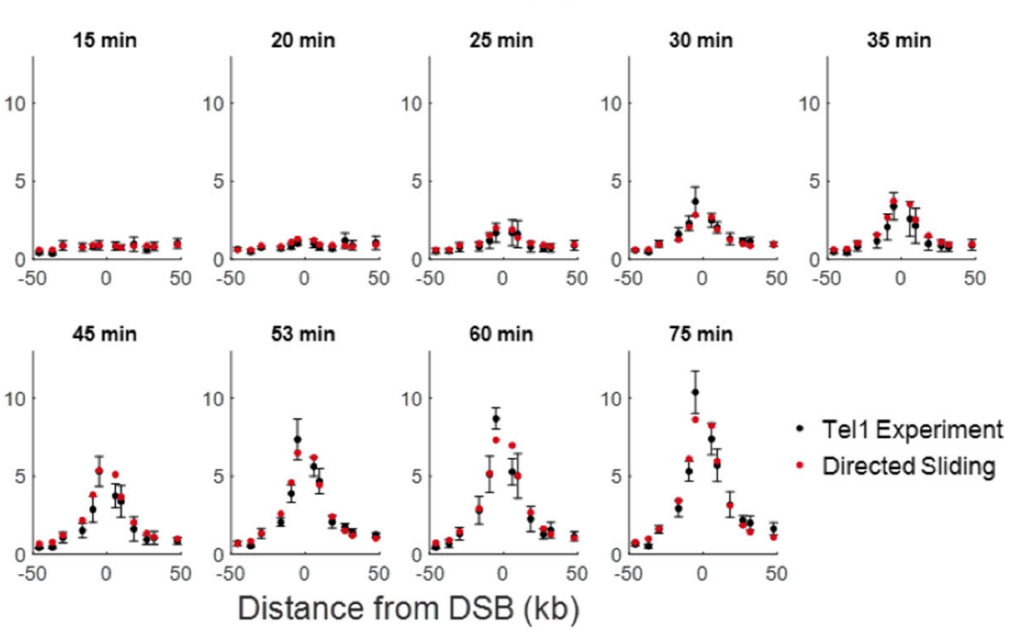

\section{Figure 5: Comparison of experimental $\gamma$-H2AX profiles to the most likely theoretical spreading distributions}

Comparison of experimental $\gamma-\mathrm{H} 2 \mathrm{AX}$ profiles (black) to theoretical $\gamma-\mathrm{H} 2 \mathrm{AX}$ distributions for the best model. Experimental and theoretical curves for 3D diffusion (blue) by Mec1 phosphorylation are shown in (A) and experimental and theoretical curves for directed sliding (red) by Tell are shown in (B). The experimental error bars represent the standard error of the mean from $\mathrm{n} \geq 3$ measurements. The theory curves are plotted using parameters values shown in Table 2. Due to concerns about the reduction in $\gamma$-H2AX signal from resection close to the DSB, the $-1.6 \mathrm{~kb}$ and $-2.1 \mathrm{~kb}$ data points (not shown) were excluded when performing fits to the experimental data. For the plots in $\mathbf{A}$ and $\mathbf{B}$, our theoretical predictions start from the same background levels as in the experimental data. 

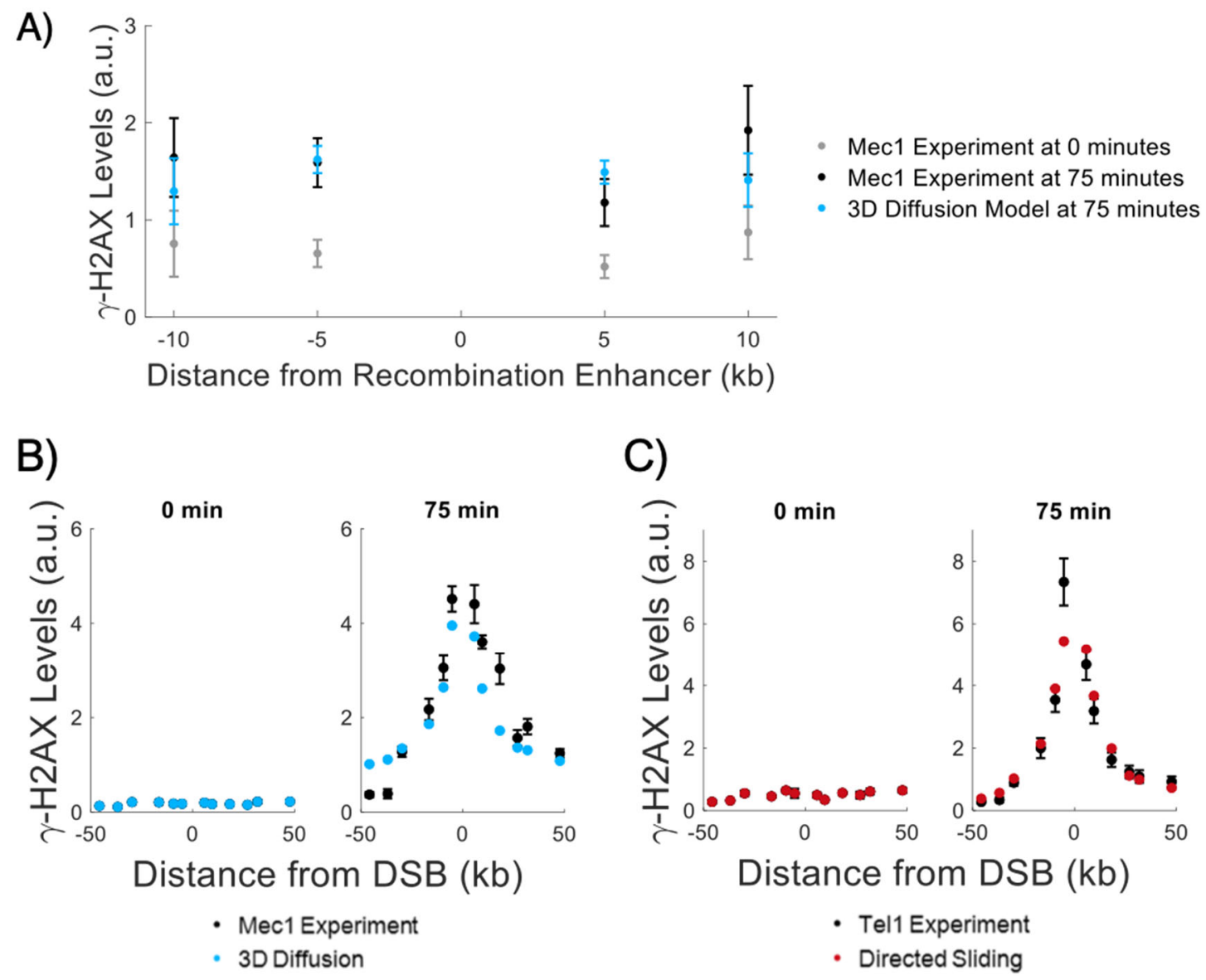

Figure 6: Comparison of experimental $\gamma-\mathrm{H} 2 \mathrm{AX}$ profiles to theoretical predictions around $\mathrm{RE}$ and in strains in which $50 \%$ of $\mathrm{H} 2 \mathrm{~A}$ sites cannot be phosphorylated

Comparison of the 3D diffusion model (blue) with the $\gamma$-H2AX measurements around the Recombination Enhancer (RE) by Mec1 is shown in (A). The experimental data at 0 min (grey) and 75 minutes (black) are displayed along with theoretical predictions based on the 3D diffusion model (blue). Experimental error bars are the standard error of the mean. Theoretical predictions from 3D diffusion were generated using the parameters listed in Table 2 with the exception of $k_{\text {init }}=0.025 /$ minute, to account for an initial delay is RE binding, consistent with the kinetics of mating type switching as measured in Avşaroğlu et al. (24). Also, the RE-DSB interaction is assumed to occur only $19 \%$ of the time. The data point at RE was excluded from the plot since the theory does not make predictions at this point. The model starts from the same background levels as the experimental data, so the large signal to noise ratio at $0 \mathrm{~min}$ leads to large error bars at $75 \mathrm{~min}$ in the theoretical curve.

The experimentally measured $\gamma-\mathrm{H} 2 \mathrm{AX}$ distributions for the HTA-S129A mutants were averaged together for each kinase. In (B) and (C), theoretical curves were generated using the parameters listed in Table 2 for 3D diffusion by Mec1 (blue) and directed sliding by Tell (red), respectively, and overlaid onto the experimentally measured $50 \% \gamma-\mathrm{H} 2 \mathrm{AX}$ profile (black). 

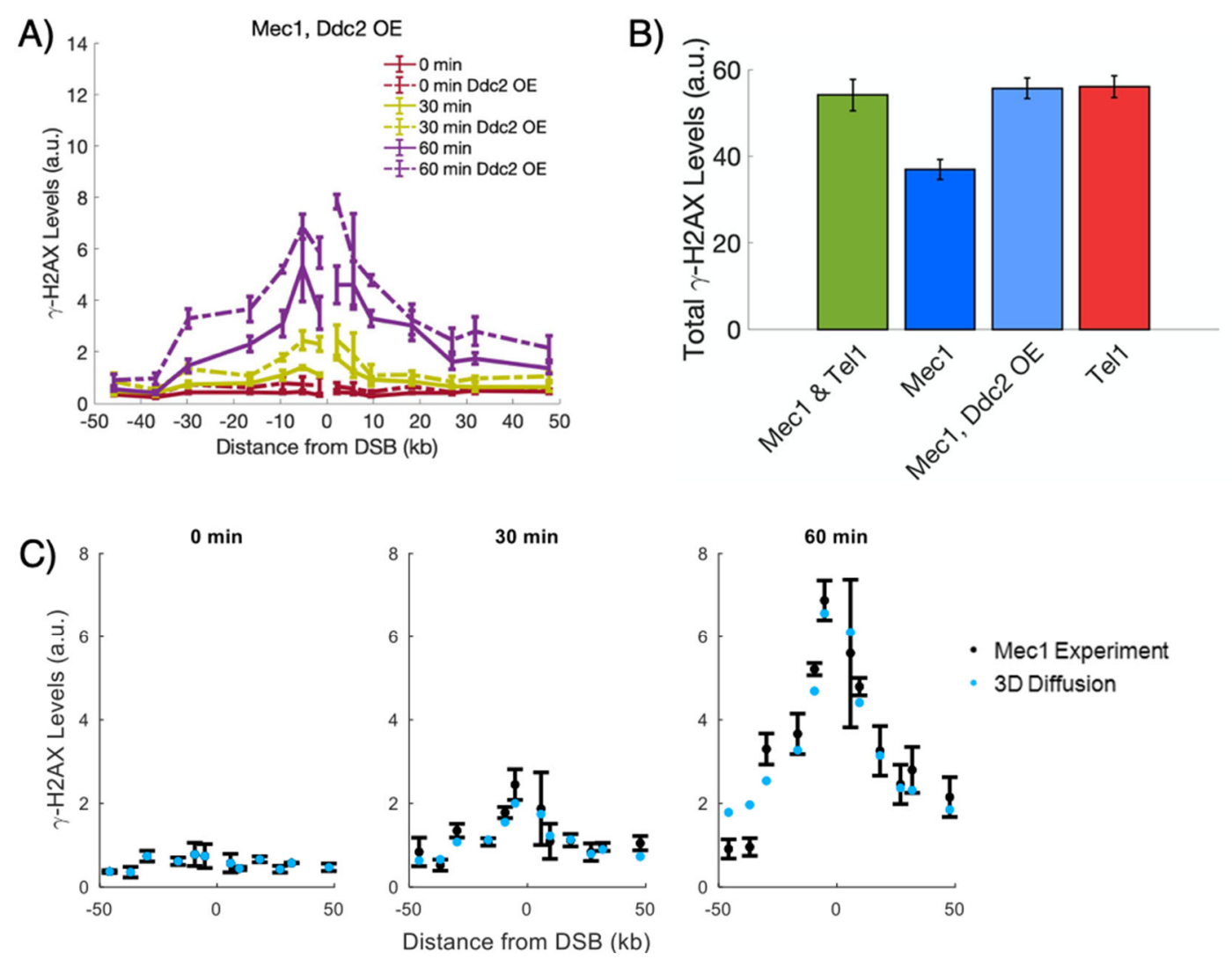

\section{Figure 7: $\gamma$-H2AX profiles by Mec1 with Ddc2 overexpression}

A) Overexpression of Ddc2 leads to increased $\gamma-\mathrm{H} 2 \mathrm{AX}$ levels by Mec1. Experimental error bars represent the standard error of the mean from $n=2$ measurements.

B) The overexpression of Ddc2 increases the total amount of phosphorylation by Mec1 (blue compared to light blue). By $60 \mathrm{~min}$, Ddc2 overexpression rises the amount of $\gamma$ $\mathrm{H} 2 \mathrm{AX}$ formed to those of Tel1-mediated phosphorylation levels (light blue compared to red). Error bars were calculated by error propagating over the error of the $\gamma-\mathrm{H} 2 \mathrm{AX}$ profiles at $60 \mathrm{~min}$ for the associated strain.

C) The prediction from the 3D diffusion model (blue) agrees with the experimental $\gamma$ H2AX levels (black) for Mec1-mediated phosphorylation in the presence of Ddc2 overexpression. Experimental error bars represent the standard error of the mean from $n=2$ measurements. Predicted levels of $\gamma-\mathrm{H} 2 \mathrm{AX}$ were calculated by treating the initial recruitment rate $k_{\text {init }}$ as a free parameter and by using the optimal parameters found for Mec1-mediated phosphorylation under wildtype levels of Ddc2 expression (Table 2). By performing Bayesian parameter estimation, we find that $k_{\text {init }}$ in the Ddc2 overexpressed case is 2.8 times higher than the optimal value in the wildtype Ddc2 case. 


\section{Tables}

\section{Table 1: $\log _{10}$ (Bayes Factor) for various model combinations}

$\log _{10}$ (Bayes Factor) is shown for each model. $\boldsymbol{A}$ ) Bayes factors were computed for the four mechanisms from Tel1-mediated phosphorylation. 1D mechanisms are much more likely than 3D mechanisms for Tel1, with directed sliding as the most likely mechanism. The Bayes factor was calculated by dividing the probability of the indicated model by the probability of the best model. For instance, -5 in the second row means that the probability of Tell undergoing 1D diffusion is $10^{-5}$ times less likely than directed sliding. $\boldsymbol{B}$ ) Bayes factors were computed for Mec1 and Tell simultaneously. The most likely model combination is Mec1 3D diffusion and Tel1 directed sliding.

A)

\begin{tabular}{cc} 
Model for Tel1 & $\log _{10}$ (Bayes Factor $)$ \\
\hline Directed Sliding & Best Model \\
1D Diffusion & -5 \\
3D Diffusion & -61 \\
Looping & -251 \\
\hline
\end{tabular}

B)

\begin{tabular}{llc}
\multicolumn{2}{c}{ Model Combination } & $\log _{10}$ (Bayes Factor) \\
\hline Mec1 - 3D Diffusion; & Tel1 - Directed Sliding & Best Model \\
Mec1 - 3D Diffusion; & Tel1 - 1D Diffusion & -6 \\
Mec1 - 1D Diffusion; & Tel1 - Directed Sliding & -12 \\
Mec1 - 1D Diffusion; & Tel1 - 1D Diffusion & -18 \\
Mec1 - Directed Sliding; & Tel1 - 1D Diffusion & -20 \\
Mec1 - Directed Sliding; & Tel1 - Directed Sliding & -35 \\
Mec1 - Looping; & Tel1 - Directed Sliding & -259 \\
Mec1 - Looping; & Tel1 - 1D Diffusion & -258 \\
\hline
\end{tabular}




\section{Table 2: Parameter values for the best models}

Displayed are the optimal parameter values obtained when Bayesian parameter estimation was performed simultaneously for Mec1 and Tel1. The 95\% CI for the parameter values are shown in parentheses. A description of the parameters can be found in Computational Methods.

\begin{tabular}{ccc} 
Tel1, Directed Sliding & Mec1, 3D Diffusion & $\begin{array}{c}\text { Parameters Shared by } \\
\text { Mec1 and Tel1 }\end{array}$ \\
\hline$k_{\text {init }}: 0.033 /$ minute $(0.0060-0.077)$ & $k_{\text {init }}: 0.089 /$ minute $(0.068-0.15)$ & $C: 26(13-280)$ \\
$k_{\text {slide }}: 3.2 \mathrm{~kb} /$ minute $(2.0-17)$ & $l: 14 \mathrm{~kb}(9.9-15)$ & $f: 0.16(0.020-0.72)$ \\
$k_{\text {off }}: 0.30 /$ minute $(0.17-1.8)$ & $\omega: 4.2 \times 10^{-3} /$ minute & $N_{H 2 A}: 6(2-6)$ \\
\hline
\end{tabular}




\section{Supplementary Information for}

\section{Yeast ATM and ATR use different mechanisms to spread histone H2A phosphorylation around a DNA double-strand break}

Kevin Li ${ }^{1}$, Gabriel Bronk ${ }^{1}$, Jane Kondev ${ }^{1}$ and James E. Haber ${ }^{2}$

1Department of Physics, Brandeis University, Waltham, MA

${ }_{2}$ Department of Biology and Rosenstiel Basic Medical Sciences Research Center, Brandeis University, Waltham, MA

Corresponding authors: James E. Haber and Jane Kondev

Email: haber@brandeis.edu, kondev@brandeis.edu

This PDF file includes:

Supplementary text

Figures S1-S14

Tables S1-S5

Description of Dataset S1

References for Supplementary Information

Other supplementary materials for this manuscript:

Dataset S1 


\section{Table of Contents}

Supplementary Text.......................................................

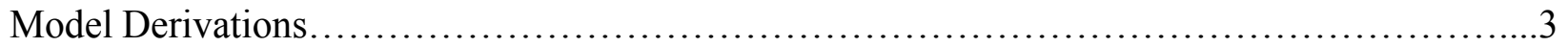

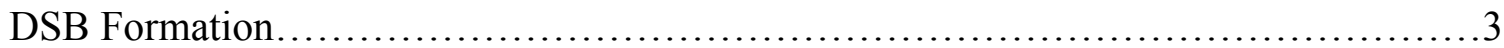

Directed Sliding Model Derivation..........................................

Looping Model Derivation................................................... 7

1D Diffusion Model Simulation.................................................. 12

3D Diffusion Model Derivation...........................................14

Modeling Phosphorylation Spreading Around the Recombination Enhancer............16

Some Models Require Kinase Activation at the DSB............................ 17

A Quantitative Model of ChIP................................................ 18

Basal $\gamma$-H2AX Levels are Incorporated into the Predicted ChIP Signals................21

A Quantitative Model of ChIP for the Directed Sliding Model........................22

Bayes Factor Calculation..................................................... 22

Supplemental Figures....................................................... 26

Supplemental Tables.......................................................4 40

Description of Supplemental Dataset .......................................... 46

References for Supplementary Information....................................47 


\section{Supplementary Text}

\section{Model Derivations}

All models are derived below and plotted in Figures S3-S14.

\section{DSB Formation}

All models begin with the formation of the DSB. We experimentally measure the cumulative probability distribution of the DSB formation time (Figure S1), and in all models we use this distribution by approximating it as a 12 minute lag time followed by the formation of the DSB at a rate of $k_{D S B}=0.08 /$ minute.

\section{Directed Sliding Model Derivation}

\section{Derivation Assuming a Kinase is at the Break Site at Time 0}

We first derive a very simple model (in the next section we go on to a more detailed model, which is the one we compare to the experimental data). We model the chromosome as a 1D lattice of H2As: the H2As are at positions $1,2,3 \ldots$ and the break site is at position 0 . The kinase is at position 0 at time 0 and then stochastically steps from one position to the next at a rate $k_{\text {slide. At }}$ time $t$, the probability that the kinase is at position $i$ is $\left(\left(k_{\text {slide }} t\right)^{i} e^{-k_{\text {slide }} t}\right) / i$ !. This is simply the Poisson distribution.

Next we include the process of the kinase falling off the chromosome at a rate $k_{\text {off }}$, regardless of where on the chromosome the kinase is located. $e^{-k_{\text {off }} t}$ is the probability that the kinase is still on the chromosome at time $t . P_{\text {kinase }}(i, t)$, the joint probability that the kinase is still on the chromosome AND is at the $i$-th $\mathrm{H} 2 \mathrm{~A}$ at time $t$, is therefore given by the product of the Poisson distribution and $e^{-k_{\text {off }} t}$ :

$$
P_{\text {kinase }}(i, t)=\frac{\left(k_{\text {slide }} t\right)^{i} e^{-k_{\text {slide }} t}}{i !} e^{-k_{\text {off }} t}
$$


$P_{\text {phos }}(i, t)$, the probability that the $\mathrm{H} 2 \mathrm{~A}$ at position $i$ has been phosphorylated by time $t$, is given by

$$
P_{\text {phos }}(i, t)=\sum_{j=i+1}^{\infty} P_{\text {kinase }}(j, t)+\int_{0}^{t} k_{\text {off }} \sum_{j=i+1}^{\infty} P_{\text {kinase }}(j, z) d z
$$

This formula follows from the assumption that the kinase phosphorylates every $\mathrm{H} 2 \mathrm{~A}$ that it comes to. Hence, if at time $t$ the kinase is currently on an $\mathrm{H} 2 \mathrm{~A}$ at any position $j>i$, then we know that the kinase has already been to the $i$-th $\mathrm{H} 2 \mathrm{~A}$ and has already phosphorylated it. The first term in equation (2) is the probability that at time $t$ the kinase is currently at any location beyond position $i$. (The summation goes to infinity for mathematical simplicity. Because $P_{\text {kinase }}(j, t)$ is small at large $j$, there would be little change if we used a summation limit equal to the actual number of H2As in the chromosome). Similarly, if the kinase has fallen off the chromosome from any position $j>i$ at any time before $t$, then we know that the $i$-th H2A has already been phosphorylated. The second term in equation (2) is the probability that the kinase has fallen off the chromosome from anywhere beyond position $i$ at any time before time $t$.

Next we simplify the summation term in equation (2):

$$
\begin{gathered}
\sum_{j=i+1}^{\infty} P_{\text {kinase }}(j, t) \\
=e^{-k_{\text {off }} t} \sum_{j=i+1}^{\infty}\left(\frac{\left(k_{\text {slide }} t\right)^{j} e^{-k_{\text {slide }} t}}{j !}\right) \\
=e^{-k_{\text {off }} t}\left(1-\sum_{j=0}^{i}\left(\frac{\left(k_{\text {slide }} t\right)^{j} e^{-k_{\text {slide }} t}}{j !}\right)\right) \\
=e^{-k_{\text {off }} t} \frac{\gamma\left(i+1, k_{\text {slide }} t\right)}{\Gamma(i+1)}
\end{gathered}
$$

where $\Gamma$ is the complete gamma function, and $\gamma$ is the lower incomplete gamma function. Plugging expression (3) into equation (2), we obtain

$$
P_{\text {phos }}(i, t)=e^{-k_{\text {off }} t} \frac{\gamma\left(i+1, k_{\text {slide }} t\right)}{\Gamma(i+1)}+\int_{0}^{t} k_{\text {off }} e^{-k_{\text {off }} z} \frac{\gamma\left(i+1, k_{\text {slide }} z\right)}{\Gamma(i+1)} d z
$$

After evaluating the integral and simplifying, we obtain 


$$
P_{\text {phos }}(i, t)=\frac{(1-q)^{i+1}}{\Gamma(i+1)}\left[i \gamma\left(i, k_{\text {move }} t\right)-\left[k_{\text {move }} t\right]^{i} e^{-k_{\text {move }} t}\right]
$$

where $q=\frac{k_{\text {off }}}{k_{\text {slide }}+k_{\text {off }}}$ and $k_{\text {move }}=k_{\text {slide }}+k_{\text {off }}$.

\section{Derivation that Incorporates the Variability in the Timing of DSB Formation and Arrival of the Kinase to the Break Site}

Until this point, we have said that at $t=0$ the kinase is at the break site and begins sliding along the chromosome. However, in different cells in the yeast population, the kinase starts sliding at different times (this will be detailed below). For an individual yeast cell, let $\tau$ be the time when the kinase begins the sliding process - i.e. the kinase does exactly what we described and derived so far, but beginning at time $\tau$ rather than time 0 . Thus, we write $P_{\text {phos }}(i, t \mid \tau)$, which is the probability that the $\mathrm{H} 2 \mathrm{~A}$ at position $i$ has been phosphorylated by time $t$, given that the kinase began the sliding process at time $\tau$ :

$$
P_{\text {phos }}(i, t \mid \tau)= \begin{cases}\frac{(1-q)^{i+1}}{\Gamma(i+1)}\left[i \gamma\left(i, k_{\text {move }}(t-\tau)\right)-\left[k_{\text {move }}(t-\tau)\right]^{i} e^{-k_{\text {move }}(t-\tau)}\right], & \text { when } t \geq \tau \\ 0, & \text { when } t<\tau\end{cases}
$$

The sliding start time is stochastic and is described by the probability density function $\operatorname{PDF}(\tau)$, which is derived in the following manner: The chromosome begins intact, which we call state I. The DSB forms at a rate $k_{D S B}$ : the chromosome is said to be in state II when there is a DSB but the kinase is not yet sliding. We make the assumption that once a yeast cell has a DSB, there is a single rate-limiting step described by the rate $k_{\text {init }}$ that determines how soon the kinase initiates the sliding process (i.e. how soon the kinase arrives to the break site and then leaves the break site to slide along the chromosome). As soon as the kinase begins the sliding process, the chromosome is said to be in state III. Thus, $k_{D S B}$ is the transition rate from state I to state II, and $k_{\text {init }}$ is the transition rate from state II to III; this is captured by the coupled differential equations and initial conditions (7). (To simplify the derivation of the sliding model, in each yeast cell we say that only one copy of the kinase arrives at the break site; additional copies would have little effect on the predicted $\gamma$ - 
$\mathrm{H} 2 \mathrm{AX}$ profile because this derivation assumes that the kinase phosphorylates every $\mathrm{H} 2 \mathrm{~A}$ that it comes to). Let the functions $I(t), I I(t)$, and $I I I(t)$ represent the probabilities of being in states I, II, and III, respectively. We have

$$
\begin{array}{lr}
\frac{d I(t)}{d t}=-k_{D S B} I(t) & I(0)=1 \\
\frac{d I I(t)}{d t}=k_{D S B} I(t)-k_{\text {init }} I I(t) & I I(0)=0 \\
\frac{d I I I(t)}{d t}=k_{\text {init }} I I(t) & I I I(0)=0
\end{array}
$$

$\operatorname{PDF}(\tau)$ is the probability per unit time that a cell will enter the state in which the kinase is sliding (i.e. state III). Therefore, $\operatorname{PDF}(\tau)=\frac{d I I I}{d t}$. Solving equations (7) we obtain

$$
P D F(\tau)=\frac{k_{\text {init }} k_{D S B}}{k_{\text {init }}-k_{D S B}}\left(e^{-k_{D S B} \tau}-e^{-k_{\text {init }} \tau}\right)
$$

We calculate $P_{\text {phos }}(i, t)$, the probability that the $\mathrm{H} 2 \mathrm{~A}$ at position $i$ has been phosphorylated by time $t$, where the time when the kinase begins the sliding process is distributed according to equation (8). $P_{\text {phos }}(i, t)$ is given by the integral of the product of equations (5) and (8):

$$
P_{p h o s}(i, t)=\int_{0}^{t} P_{p h o s}(i, t \mid \tau) P D F(\tau) d \tau
$$

Evaluating this integral gives

$$
\begin{aligned}
P_{\text {phos }}(i, t)= & \frac{(1-q)^{i+1}}{k_{\text {init }}-k_{D S B}}\left[k _ { \text { init } } \left(\frac{\gamma\left(i, k_{\text {move }} t\right)}{\Gamma(i)}+\frac{e^{-k_{\text {move }} t^{i-1} k_{\text {move }}{ }^{i}}}{\Gamma(i)\left(k_{\text {move }}-k_{D S B}\right)}\right.\right. \\
& \left.-\frac{e^{-k_{D S B} t} k_{\text {move }}{ }^{i}}{\left(k_{\text {move }}-k_{D S B}\right)^{i}}\left[\frac{\gamma\left(i+1,\left(k_{\text {move }}-k_{D S B}\right) t\right) k_{D S B}}{\Gamma(i+1)\left(k_{\text {move }}-k_{D S B}\right)}+\frac{\gamma\left(i-1,\left(k_{\text {move }}-k_{D S B}\right) t\right)}{\Gamma(i-1)}\right]\right) \\
& -k_{D S B}\left(\frac{\gamma\left(i, k_{\text {move }} t\right)}{\Gamma(i)}+\frac{e^{-k_{\text {move }} t} t^{i-1} k_{\text {move }}{ }^{i}}{\Gamma(i)\left(k_{\text {move }}-k_{\text {init }}\right)}\right. \\
& \left.\left.-\frac{e^{-k_{\text {init }}{ }^{t} k_{\text {move }}}}{\left(k_{\text {move }}-k_{\text {init }}\right)^{i}}\left[\frac{\gamma\left(i+1,\left(k_{\text {move }}-k_{\text {init }}\right) t\right) k_{\text {init }}}{\Gamma(i+1)\left(k_{\text {move }}-k_{\text {init }}\right)}+\frac{\gamma\left(i-1,\left(k_{\text {move }}-k_{\text {init }}\right) t\right)}{\Gamma(i-1)}\right]\right)\right]
\end{aligned}
$$




\section{Looping Model Derivation}

We model the chromosome as an unconfined worm-like chain at thermodynamic equilibrium. It is not necessary to include confinement by the nuclear envelope because we are only interested in the loci where phosphorylation occurs, which are $50 \mathrm{~kb}$ or less from the DSB. At thermodynamic equilibrium, there is a roughly $0.4 \mu \mathrm{m}$ RMSD between two loci separated by $50 \mathrm{~kb}$ of chromatin (we estimated this based on the persistence length and compaction of the chromosome from Arbona et al. (1) and used the formula for $R$ in the Supplementary Information, 3D Diffusion Model Derivation). The nucleus has a diameter of $2 \mu \mathrm{m}$, which is much larger than the $0.4 \mu \mathrm{m}$ RMSD, so loci at $50 \mathrm{~kb}$ from the DSB are not confined by the nuclear envelope to stay in closer proximity to the DSB.

Using the equilibrium distribution of worm-like chain conformations is justified because we estimate that loci $50 \mathrm{~kb}$ away from each other should encounter each other every few minutes (every few seconds for loci $10 \mathrm{~kb}$ away from each other), which is much faster than the few tensof-minutes time scale over which $\mathrm{H} 2 \mathrm{~A}$ phosphorylation occurs. We can estimate the contact frequency between two loci because we know the root mean squared distance $R$ at equilibrium for the two loci. We also know the time $t$ for a locus to diffuse a distance equal to $R-$ this is obtained by inspecting the mean squared displacement (MSD) vs. time plot in Hajjoul et al. (2). Counterintuitively, $t$ is equivalent to the time it takes for the two loci to come in contact. This is because chromatin undergoes subdiffusion, and any object moving subdiffusively thoroughly explores its space $(3)$ - i.e. in the time $t$, the locus thoroughly explores a sphere of radius $R$. Therefore, loci within a distance $R$ of each other will come in contact during the time $t$.

Now we introduce the formula for the worm-like chain looping probability. Let $l$ be the Kuhn length of the worm-like chain and $x$ be the contour length between two particular locations on the chain. $n=x / l$ is therefore the number of Kuhn lengths between the two locations. $P_{\text {loop }}$ is the fraction of chain conformations that have these two locations in physical contact. To be considered in physical contact, we say that the 3D distance between the two locations must be less than $\mathrm{g}$, where $\mathrm{g}$ is a small fraction of a Kuhn length ( $\mathrm{g}$ is unitless because it is a fraction). $P_{\text {loop }}$ is given by 


$$
P_{\text {loop }}(n)=1.34 g^{3} n^{-3 / 2} e^{-2 n^{-2}}
$$

Note that equation (11) is an approximation - it does not predict $P_{\text {loop }}$ exactly. Nevertheless, we compared equation (11) to worm-like chain simulations, and we found that the formula is accurate (i.e. has a maximum error of about $10 \%$ ) for $1 \leq n \leq 20$ and $g \leq 0.1$. Equation (11) is based on ref. (4).

Now we incorporate the kinase into the model. Suppose the kinase is at the break site, and consider an H2A that is separated from the DSB by $n$ Kuhn lengths of chromatin. $P_{\text {loop }}(n)$ is therefore the probability that the chromosome is in a looped conformation in which the kinase and $\mathrm{H} 2 \mathrm{~A}$ are in physical contact. For H2A phosphorylation to occur, not only is this contact necessary but the kinase must also be in the necessary orientation. Let $\epsilon$ be the fraction of orientations that can result in phosphorylation, given that the $\mathrm{H} 2 \mathrm{~A}$ and kinase are in contact. Let $k_{c a t}$ be the rate at which the kinase phosphorylates the $\mathrm{H} 2 \mathrm{~A}$, given that the kinase is in contact with the $\mathrm{H} 2 \mathrm{~A}$ and is in the necessary orientation. Therefore, the rate of phosphorylation of the $\mathrm{H} 2 \mathrm{~A}$ is

$$
k_{\text {phos }, L M}(n)=k_{\text {cat }} \in P_{\text {loop }}(n)
$$

where the "LM" subscript stands for the looping model. Substitute equation (11) into equation (12), and replace $n$ with $x / l$, and we obtain

$$
\begin{aligned}
k_{p h o s, L M}(x) & =1.34 k_{c a t} \epsilon g^{3}(l / x)^{3 / 2} e^{-2(l / x)^{2}} \\
& =\varphi(l / x)^{3 / 2} e^{-2(l / x)^{2}}
\end{aligned}
$$

where $\varphi=1.34 k_{\text {cat }} \epsilon g^{3}$. Note that for $x$ and $l$ we use units of kilobase pairs because in our experiments we know the number of kilobase pairs between the DSB and our measured loci.

We construct the model to be consistent with ChIP measurements of Tel1 and Mec1 at the break site, which show their levels increase linearly with time $(15,29)$. These measurements could indicate two different dynamics. First, they could imply that there is an increasing number of kinases at the break site, and higher numbers of kinases could result in proportionally higher rates of H2A phosphorylation (this model was not considered in the main text). The phosphorylation 
rate is therefore the product of $k_{p h o s, L M}(x)$ and the number of kinase copies on the DSB. We will refer to this as the "linearly increasing phosphorylation rate."

Alternatively, even with multiple copies of kinases bound near the break site, it is possible that only one or two copies perform phosphorylation. (Hypothetical scenarios that would cause this include these possible examples: there is a row of kinases near the break site, but only the kinase precisely at the DSB is activated, or steric hinderance causes only the kinase copies at the ends of the row to have their active sites accessible to H2As). Thus, in each cell, once a kinase has arrived to the DSB, there is simply a constant rate of $\mathrm{H} 2 \mathrm{~A}$ phosphorylation given by $k_{\text {phos, LM }}(x)$ (this model was considered in the main text). We will refer to this as the "constant phosphorylation rate." The constant and linearly increasing phosphorylation rates are the two extremes in possible dynamics; intermediate dynamics could occur - i.e. phosphorylation rates that increase in a sublinear manner. We only examine the two extremes because they yield the greatest difference in predicted $\gamma-\mathrm{H} 2 \mathrm{AX}$ profiles. We will begin with a derivation that involves the constant phosphorylation rate.

\section{Looping Model with a Constant Phosphorylation Rate}

This is the model discussed in the main text.

When a kinase is present on the break site, it phosphorylates $\mathrm{H} 2 \mathrm{As}$ at a rate $k_{p h o s, L M}(x)$, so we have

$$
\begin{aligned}
& \frac{d P_{\text {phos }}(x, t \mid \tau)}{d t}=\left(1-P_{\text {phos }}(x, t \mid \tau)\right) k_{\text {phos }, L M}(x) \\
& P_{\text {phos }}(x, t \mid \tau)=0 \text { for } t<\tau
\end{aligned}
$$

where $P_{\text {phos }}(x, t \mid \tau)$ is the probability that the H2A at $x$ kilobase pairs from the break site has been phosphorylated by time $t$, given that the kinase arrived to the break site at time $\tau$. (The bottom equation of equations (14) states that no phosphorylation takes place until the kinase arrives to the break site). Solving equations (14) gives

$$
P_{\text {phos }}(x, t \mid \tau)= \begin{cases}1-e^{-k_{\text {phos }, L M}(x)(t-\tau)}, & \text { when } t \geq \tau \\ 0, & \text { when } t<\tau\end{cases}
$$


Recall that in the "constant phosphorylation rate" version of the looping model, we only consider a single copy of the kinase at the break site because we assume that additional copies of the kinase would not change the phosphorylation rate. In different cells in the yeast population, the single copy of the kinase arrives to the break site at different times. To take this into account, we perform the same derivation that is in the directed sliding model's section entitled "Derivation that Incorporates the Variability in the Timing of DSB Formation and Arrival of the Kinase to the Break Site:" $P_{p h o s}(x, t)$, the probability that the H2A at $x$ kilobase pairs from the break site has been phosphorylated by time $t$, is given by

$$
P_{\text {phos }}(x, t)=\int_{0}^{t} P_{\text {phos }}(x, t \mid \tau) P D F(\tau) d \tau
$$

where $P_{\text {phos }}(x, t \mid \tau)$ is given by equation (15) and $\operatorname{PDF}(\tau)$ is given by equation (8). Note that when using equation (8) for the looping model, $k_{\text {init }}$ indicates the rate at which the kinase arrives to the break site (note that this differs from the definition of $k_{\text {init }}$ used in the sliding model because in the sliding model $k_{\text {init }}$ involves kinase arrival to the break site AND the process of leaving the break site to begin sliding). Upon carrying out the integration in equation (16), we obtain

$$
\begin{aligned}
P_{\text {phos }}(x, t) & =1+\frac{k_{D S B} k_{\text {phos }, L M}(x) e^{-k_{\text {init }} t}}{\left(k_{D S B}-k_{\text {init }}\right)\left(k_{\text {init }}-k_{\text {phos }, L M}(x)\right)} \\
+ & \frac{k_{D S B} k_{\text {init }} e^{-k_{p h o s, L M}(x) t}}{\left(k_{\text {phos }, L M}(x)-k_{D S B}\right)\left(k_{\text {init }}-k_{\text {phos }, L M}(x)\right)}+\frac{k_{\text {init }} k_{p h o s, L M}(x) e^{-k_{D S B} t}}{\left(k_{\text {init }}-k_{D S B}\right)\left(k_{D S B}-k_{p h o s, L M}(x)\right)} .
\end{aligned}
$$

\section{Looping Model with a Linearly Increasing Phosphorylation Rate}

This model is not included in the main text because the Bayes factor calculations demonstrate that it is worse than the model with the constant phosphorylation rate. In this version of the looping model, the phosphorylation rate is proportional to the number of copies of the kinase that are on the break site. Let $M(t \mid \delta)$ be the average number of copies of the kinase on the break site at time $\mathrm{t}$, given that the DSB formed at time $\delta$. Therefore, we have 


$$
\begin{aligned}
& \frac{d P_{p h o s}(x, t \mid \delta)}{d t}=M(t \mid \delta) k_{p h o s, L M}(x)\left(1-P_{p h o s}(x, t \mid \delta)\right) \\
& P_{p h o s}(x, t \mid \delta)=0 \text { for } t<\delta
\end{aligned}
$$

where $P_{\text {phos }}(x, t \mid \delta)$ is the probability that the H2A at $x$ kilobase pairs from the break site has been phosphorylated by time $t$, given that the DSB formed at time $\delta$. (For simplicity, we obtain an approximate solution by using the mean number of kinase copies on the break site $M(t \mid \delta)$, ignoring the variance in the numbers of kinase copies.)

The average number of kinase copies at the break site is linear with time (i.e. kinases arrive with a constant rate $\left.k_{\text {init }}\right)$, so $M(t \mid \delta)=(t-\delta) k_{\text {init }}$. Solving equations (18) using this expression for $M(t \mid \delta)$ gives

$$
P_{\text {phos }}(x, t \mid \delta)= \begin{cases}1-e^{-\frac{k_{\text {init }} k_{\text {phos }, L M}(x)(t-\delta)^{2}}{2}}, & \text { when } t \geq \delta \\ 0, & \text { when } t<\delta\end{cases}
$$

Next we take into account that $\delta$ varies across yeast cells in the population. Because the DSB forms at a constant rate $k_{D S B}$, there is an exponential distribution of waiting times for DSB formation, given by the following probability density function for $\delta$ :

$$
P D F(\delta)=k_{D S B} e^{-k_{D S B} \delta}
$$

$P_{\text {phos }}(x, t)$, the probability that the $\mathrm{H} 2 \mathrm{~A}$ at $x$ kilobase pairs from the break site has been phosphorylated by time $t$, is given by

$$
P_{\text {phos }}(x, t)=\int_{0}^{t} P_{\text {phos }}(x, t \mid \delta) P D F(\delta) d \delta
$$

We plug equations (19) and (20) into equation (21) and evaluate the integral numerically.

Note that in equation (19), $k_{\text {init }}$ and $k_{p h o s, L M}(x)$ never appear separately: they are always multiplied together. Using equation (13), we have $k_{\text {init }} k_{\text {phos, LM }}(x)=k_{\text {init }} \varphi(l / x)^{3 / 2} e^{-2(l / x)^{2}}$, so we define the parameter $\psi=k_{\text {init }} \varphi$ (i.e. $k_{\text {init }}$ and $\varphi$ are not separate parameters in the "Linearly 
Increasing Phosphorylation Rate" version of the looping model). Therefore, this version of the looping model has only two parameters: $\psi$, and $l$.

\section{D Diffusion Model Simulation}

Here we describe additional details of the Gillespie simulations for 1D diffusion that were not discussed in the Computational Methods section of the main text. As previously mentioned, we construct all of our models to be consistent with ChIP measurements of Mec1 and Tell at the break site, which show Mec1 and Tel1 levels that increase linearly with time. In the 1D diffusion model, linearly increasing kinase levels could be achieved through one of two simple mechanisms, one in which there is a constant rate at which kinases initiate 1D diffusion, and a mechanism in which the rate of diffusion initiation increases linearly with time. The former mechanism is discussed in the main text, while the latter mechanism is not since our Bayes factor calculation demonstrates that the former is more likely to be correct. We will now describe how these mechanisms give rise to linearly increasing kinase levels at the break site and will discuss how these mechanisms are implemented in our simulations.

We begin by describing the mechanism with a constant rate of diffusion initiation. Let $k_{\text {arriveDSB be }}$ the rate at which a kinase arrives to the break site and kleaveDSB be the rate at which a kinase leaves the break site to start diffusing along the chromosome. In the mechanism with a constant rate of diffusion initiation, only one kinase copy can leave the break site at a time. (This could happen if the copies of the kinase line up in a row close to the break site, such that the one copy furthest from the DSB is physically blocking the other copies from being able to slide away from the break site). In this scenario, if $k_{\text {arriveDSB }}>k_{\text {leaveDSB }}$, then copies of the kinase will build up at the break site linearly with time: we will have $M(t)=\left(k_{\text {arriveDSB }}-k_{\text {leaveDSB }}\right)(t-\delta)$, where $\delta$ is the time when the DSB forms, and $M(t)$ is the average number of kinases on the break site $(t-\delta)$ minutes after the formation of the DSB.

We perform this simulation by having a single rate-limiting step described by the rate $k_{\text {init }}$, where $k_{\text {init }}$ is the rate at which a kinase copy arrives to the break site and then departs the break site to 
diffuse along the chromosome. (Note that it is only necessary to use this single rate rather than the two rates $k_{\text {arriveDSB }}$ and $k_{\text {leaveDSB }}$ : using the single rate becomes equivalent to using two rates if $k_{\text {arriveDSB }}$ is fast. If $k_{\text {arriveDSB }}$ is slow, then the situation becomes equivalent to the mechanism in which the rate of diffusion initiation increases linearly with time, which is discussed in the next paragraph.) Throughout the entire duration of the simulation, kinase copies continue to start at rate $k_{\text {init }}$. Therefore, in our simulations there can be many kinase copies diffusing concurrently on the chromosome.

In the mechanism in which the rate of diffusion initiation increases linearly with time, the kinases do not block each other, but rather all kinases on the break site are capable of sliding away from the break site. In this case, the number of kinases that leave the break site per unit time is $M(t) k_{\text {leaveDSB }}$. If $k_{\text {arriveDSB }} \gg M(t) k_{\text {leaveDSB }}$, then copies of the kinase will build up at the break site linearly with time: we will have $M(t)=k_{\text {arrive } D S B}(t-\delta)$. The number of kinases that leave the break site per unit time will therefore be $k_{\text {leaveDSB }} k_{\text {arriveDSB }}(t-\delta)$. In other words, unlike in the first mechanism where there is a constant rate of kinase copies starting to diffuse along the chromosome, in this mechanism the rate of kinase copies starting to diffuse grows linearly with time. We implement this mechanism's simulation by having each kinase copy arrive to the break site at a rate $k_{\text {arriveDSB }}$ and leave the break site at a rate $k_{\text {leaveDSB }}$. Note, however, that these rates are not separate parameters because the dynamics are governed by the product $k_{\text {leaveDSB }} k_{\text {arriveDSB }}$, so when we vary parameter sets, we only vary $k_{\text {arriveDSB, }}$ and we simply set $k_{\text {leaveDSB }}=0.0005 /$ minute (we chose this value since it is slow enough for there to be linear growth in the number of kinases at the break site over several hours). When we report parameter values, we report $z$, where $z=k_{\text {leaveDSB }} k_{\text {arriveDSB }}$. Kinase copies begin diffusing throughout the entire duration of the simulation, so there can be many kinase copies diffusing concurrently on the chromosome. For simplicity, in all 1D diffusion simulations we assume the kinases do not interact with each other (i.e. no traffic jams); to avoid the regime where traffic jams would become likely, we limit how large $k_{\text {init }}$ and $z$ can be in order to limit the total number of kinases along the chromosome. 


\section{D Diffusion Model Derivation}

We treat the kinase as a randomly diffusing particle and each H2A histone as a spherical target of radius $a$. Given infinite time, a particle diffusing without constraints can either hit the target or diffuse infinitely far away without ever hitting the target - the probability that the particle hits the target is $P_{\text {contact }}=a / R(7)$, where $R$ is the $3 \mathrm{D}$ distance between the target and particle at the beginning of the particle's trajectory. The infinite time assumption approximates our situation because the hour time scale of phosphorylation is much longer than the seconds time scale presumably required for proteins to diffuse across the nucleus (see ref. (8) for diffusion in the nucleus of human cells). The assumption of no constraints can be applied to our system despite the presence of the nuclear envelope. Confinement by the nuclear membrane keeps the kinases in the nucleus, so the kinases presumably continue phosphorylating H2As, resulting in a background $\gamma$-H2AX signal that is approximately the same for all H2As. It is a reasonable assumption that the kinase's catalytic rate is slow enough that over the time scale of the experiment, the kinases would only have time to phosphorylate a small fraction of the H2As throughout the nucleus. The background signal would therefore be minimal and can be ignored, allowing us to simply have $P_{\text {contact }}=a / R$. (Alternatively, it is also possible that the background signal is not negligible, but having a significant background signal would make the $3 \mathrm{D}$ diffusion model not match the experimental $\gamma-\mathrm{H} 2 \mathrm{AX}$ profiles, so we do not consider this scenario).

The kinase starts its trajectory at the break site, and the target is an $\mathrm{H} 2 \mathrm{~A}$ located $x \mathrm{~kb}$ from the DSB. The distance $R$ is taken to be the root mean squared distance from the H2A to the DSB, given by a worm-like chain treatment of the chromatin at thermodynamic equilibrium: $R=\frac{l}{\eta} \sqrt{\frac{e^{-2 x / l}+2 x / l-1}{2}}$, where $l$ is the chromatin's Kuhn length, and $\eta$ is the chromatin compaction, the number of kilobases of DNA per nanometer of chromatin fiber. Therefore, $P_{\text {contact }}(x, l)=\frac{\sqrt{2} a \eta}{l \sqrt{e^{-2 x / l}+2 x / l-1}}$. According to $P_{\text {contact }}, \mathrm{H} 2$ As further from the DSB are less likely to be contacted by the kinase. 
We assume many copies of the kinase come on and off the DSB, where the rate of leaving the $\mathrm{DSB}$ is $k_{\text {leaveDSB }}$. Therefore, an $\mathrm{H} 2 \mathrm{~A}$ located $x \mathrm{~kb}$ from the DSB is hit by kinases at a rate $P_{\text {contact }}(x, l) k_{\text {leaveDSB }}$. If $v$ is the fraction of contacts that results in phosphorylation, the rate at which the $\mathrm{H} 2 \mathrm{~A}$ is phosphorylated is $k_{\text {phos }, 3 D D M}(x)=v P_{\text {contact }}(x, l) k_{\text {leaveDSB }}$ (the subscript "3DDM" stands for the 3D diffusion model). Plugging in the expression for $P_{\text {contact }}$, we have

$$
k_{p h o s, 3 D D M}(x)=\frac{\omega}{\sqrt{e^{-2 x / l}+2 x / l-1}}
$$

where $\omega=\frac{\sqrt{2} v a \eta}{l} k_{\text {leaveDSB }}$.

There are two versions of the 3D diffusion model: one with a constant rate of diffusion initiation (which is the model discussed in the main text), and a version with a linearly increasing rate of diffusion initiation. The rationale for trying these two model variants is the same as that described in the $1 D$ Diffusion Model Simulation section above. The Bayes factor analysis determined that the $3 \mathrm{D}$ diffusion model with the constant rate is more likely to be correct than the $3 \mathrm{D}$ diffusion model with the linearly increasing rate.

The 3D diffusion model with a constant rate of diffusion initiation involves kinases leaving the break site at a constant rate and therefore phosphorylating H2As at a constant rate. This model is derived in exactly the same way as the looping model with a constant phosphorylation rate (equation (17) ), except the phosphorylation rate $k_{p h o s, L M}(x)$ is replaced by $k_{p h o s, 3 D D M}(x)$, so we obtain

$$
\begin{aligned}
P_{\text {phos }}(x, t)= & +\frac{k_{D S B} k_{p h o s, 3 D D M}(x) e^{-k_{\text {init }} t}}{\left(k_{D S B}-k_{\text {init }}\right)\left(k_{\text {init }}-k_{p h o s, 3 D D M}(x)\right)} \\
& +\frac{k_{\text {init }} k_{p h o s, 3 D D M}(x) e^{-k_{D S B} t}}{\left(k_{\text {init }}-k_{D S B}\right)\left(k_{D S B}-k_{p h o s, 3 D D M}(x)\right)} \\
& +\frac{k_{D S B} k_{\text {init }} e^{-k_{\text {phos }, 3 D D M}(x) t}}{\left(k_{\text {phos }, 3 D D M}(x)-k_{D S B}\right)\left(k_{\text {init }}-k_{p h o s, 3 D D M}(x)\right)}
\end{aligned}
$$


The 3D diffusion model with a linearly increasing rate of diffusion initiation involves kinases leaving the break site at an increasing rate. This model is derived in exactly the same way as the looping model with a linearly increasing phosphorylation rate (equations (19)-(21)), except the phosphorylation rate $k_{p h o s, L M}(x)$ is replaced by $k_{p h o s, 3 D D M}(x)$, so we have the following equations:

$$
\begin{aligned}
& P_{\text {phos }}(x, t \mid \delta)=\left\{\begin{array}{lr}
1-e^{-\frac{k_{\text {init }} k_{\text {phos }, 3 D D M}(x)(t-\delta)^{2}}{2}}, & \text { when } t \geq \delta \\
0, & \text { when } t<\delta
\end{array}\right. \\
& P D F(\delta)=k_{D S B} e^{-k_{D S B} \delta} \\
& P_{\text {phos }}(x, t)=\int_{0}^{t} P_{\text {phos }}(x, t \mid \delta) P D F(\delta) d \delta
\end{aligned}
$$

where the integral is evaluated numerically. $k_{\text {init }}$ and $k_{p h o s, 3 D D M}$ always appear multiplied together.

From equation (22), we see that $k_{\text {init }} k_{p h o s, 3 D D M}(x)=\frac{k_{\text {init }} \omega}{\sqrt{e^{-2 x / l}+2 x / l-1}}$, so we define the parameter $\zeta=k_{\text {init }} \omega$. Therefore, this version of the 3D diffusion model has only two parameters: $\zeta$, and $l$.

\section{Modeling Phosphorylation Spreading Around the Recombination Enhancer}

When predicting $\gamma$-H2AX levels near the Recombination Enhancer (RE), we take into account the protein-mediated binding of RE to the break site at the MAT locus $(23,24)$. With RE bound to the $\mathrm{DSB}, \mathrm{RE}$-adjacent loci (that are 5 or $10 \mathrm{~kb}$ from RE) are now in close spatial proximity to the DSB, so the 3D models predict that the kinases can reach the RE-adjacent sites. 1D models predict that the kinases cannot reach RE-adjacent sites because RE is located $171 \mathrm{~kb}$ away from MAT. For 3D models, we include two additional parameters in order to take into account the binding of RE to MAT. Initially there is a period when RE is not bound to the DSB at all, but then proteins at $M A T$ undergo phosphorylation at their threonine residues, which mediates the RE-MAT interaction. Therefore, we include in the model that at rate $k_{R E}$ the cell transitions to the state in which RE and the DSB are bound some fraction of the time (denoted by $F_{R E}$ ), at which point the kinase can phosphorylate RE-adjacent sites. The rate of phosphorylation for an $\mathrm{H} 2 \mathrm{~A}$ that is $x \mathrm{~kb}$ from $\mathrm{RE}$ is assumed to equal the phosphorylation rate for an $\mathrm{H} 2 \mathrm{~A}$ that is $x \mathrm{~kb}$ from the DSB 
multiplied by $F_{R E}$. In other words, for the RE-adjacent sites, $P_{\text {contact }}(x, l)$ in the $3 \mathrm{D}$ diffusion model and $P_{\text {loop }}(x, l)$ in the looping model are multiplied by $F_{R E}$ because the kinase only comes in contact with the RE-adjacent sites during the fraction of the time when RE and MAT are bound. The optimal values of the parameters are $F_{R E}=0.19$ and $k_{R E}=0.025 /$ minute, which are consistent with the kinetics of mating type switching as measured in Avşaroğlu et al. (10). Because $k_{R E}$ is subtantially slower than $k_{\text {init }}, k_{R E}$ is rate-limiting, so we simply treat $k_{\text {init }}$ as equal to $0.025 /$ minute in equations $(17,23)$.

\section{Some Models Require Kinase Activation at the DSB}

The 3D diffusion, 1D diffusion and directed sliding models require that the kinase becomes activated upon arrival to the break site. Without this activation, these models would not predict preferential phosphorylation of H2As close to the break site; the kinase becomes activated at the DSB and is more likely to encounter closer H2As than farther H2As. To further argue this point, consider a model in which the kinase is activated without having to encounter the DSB. At the moment when the DSB is formed, the kinase is at some arbitrary location in the nucleus, so it is equally likely to diffuse to any genetic locus and then start phosphorylating H2As from that locus (this applies for directed sliding, 1D diffusion and 3D diffusion). Simply assuming strong binding between the kinase and the break site would not result in increased phosphorylation near the DSB. Strong binding to the DSB would merely hold the kinase in place for a while - i.e. kinases that happen to come in contact with the DSB would end up wasting their time sitting on the DSB, so they would have less time to phosphorylate H2As. As previously noted, a precedent for kinase activation was found by an in vivo study of ATM, the mammalian homolog of Tel1: upon binding to the MRN complex at the break site, ATM undergoes autophosphorylation and thus becomes active (11).

Note that the 1D diffusion and directed sliding models are mathematically equivalent to models in which many copies of the kinase bind along the chromatin, each binding to the next adjacent H2A. In this case, kinase activation could take place but is not required in the model. This is because the strong binding of the kinase to the break site could serve to nucleate the formation of the row of kinases. The directed sliding model is also equivalent to a model in which the kinase remains 
bound to the break site while the nearby chromatin slides past it - e.g. this would happen if the cohesin complex binds to the DSB and extrudes chromatin through the cohesin ring, causing the DSB-bound kinase to slide past the nearby chromatin; such a model does not require kinase activation at the break site (chromatin extrusion by cohesin has been found to occur in mammals (12), though studies have not investigated whether it leads to the spreading of histone modifications).

Also note that the looping model does not require activation at the DSB: H2As are preferentially phosphorylated in the vicinity of the DSB because the kinase binds the DSB and the looping of the chromosome brings the kinase into contact with nearby H2As.

\section{A quantitative model of ChIP}

In order to quantitatively compare our phosphorylation propagation models to ChIP data, we must have a quantitative understanding of ChIP. It is not sufficient to merely have the qualitative understanding that a higher ChIP signal indicates the presence of more $\gamma$-H2AXs. Our phosphorylation propagation models predict the probability $P_{p h o s}(x, t)$ that an $\mathrm{H} 2 \mathrm{~A}$ has been phosphorylated, where $x$ is the number of kilobase pairs separating the H2A from the break site, and $t$ is the elapsed time since the addition of galactose (galactose induces production of HOendonuclease, the enzyme that forms the DSB). Measuring the ChIP signal does not directly give us the probability of phosphorylation. Rather, the ChIP signal is some function of this probability: the function $S\left(P_{\text {phos }}\right)$ will represent the ChIP signal. Below, we make a model of the ChIP process in order to derive the functional form of $S$. Having obtained the functional form of $S$, we can use it in conjunction with the $P_{p h o s}(x, t)$ predicted by each phosphorylation propagation mechanism: We plug in the predicted $P_{p h o s}(x, t)$ as the argument of $S$ to yield the predicted $\gamma$ H2AX ChIP profile $S\left(P_{\text {phos }}(x, t)\right)$ for each mechanism of phosphorylation propagation. We can then compare each mechanism's predicted $\gamma$-H2AX ChIP profile to the experimentally-measured ChIP profile. 
The experimental ChIP signal that we report is $S=280 \frac{\gamma H 2 A X \quad \text { signal }}{\text { control signal }} \cdot \gamma-H 2 A X$ signal is the qPCR signal for the DNA that has $\gamma-\mathrm{H} 2 \mathrm{AX}$ bound to it; control signal is the qPCR signal from all DNA at the same locus (i.e. all DNA regardless of whether it has $\gamma$-H2AX on it). The 280 is present because the sample for the control was diluted by a factor of 280 relative to the sample for the $\gamma$ $H 2 A X$ signal. We quantitatively model $S$ by taking into account that only some of the DNA with $\gamma$-H2AX on it is pulled down by antibodies during ChIP: let $P_{\text {pull-down }}\left(P_{\text {phos }}\right)$ be the probability of pulling down DNA at the measured locus, which depends on the $P_{p h o s}$ at that locus (discussed further below). We also take into account that aside from the pull-down, other steps (e.g. washing steps) in ChIP may reduce the recovery of DNA. Let $C$ be 280 multiplied the probability that the DNA will be recovered in all the other steps, given that it was pulled down by the antibodies. Therefore, our model for the ChIP signal is $S\left(P_{\text {phos }}\right)=C P_{\text {pull-down }}\left(P_{\text {phos }}\right)$.

Let us now quantitatively model $P_{\text {pull-down }}$. In the ChIP protocol, sonication results in DNA fragments roughly $500 \mathrm{bp}$ in length, containing multiple H2As. The DNA fragment comes to the surface or interior of a porous agarose bead, where the DNA fragment's $\gamma$-H2AXs can bind to antibodies that are attached to the bead. We make two assumptions: (1) The binding of a single antibody to a single $\gamma-\mathrm{H} 2 \mathrm{AX}$ results in the DNA fragment being pulled down. (Even if there are multiple $\gamma-\mathrm{H} 2 \mathrm{AX}$ s on a DNA fragment, only one $\gamma-\mathrm{H} 2 \mathrm{AX}$ needs to be bound by an antibody for the DNA fragment to be pulled down). Let the parameter $f$ be the probability that a particular $\gamma-$ $\mathrm{H} 2 \mathrm{AX}$ is bound by an antibody. (2) The second assumption is that the antibodies bind independently of each other - i.e. an antibody binding to one $\gamma$-H2AX has no effect on whether an antibody binds to another $\gamma-\mathrm{H} 2 \mathrm{AX}$ on the same DNA fragment.

These two assumptions are captured mathematically in the following manner: Let $N_{H 2 A}$ be the average number of phosphorylatable H2A's on a DNA fragment-e.g. $N_{H 2 A}=6$ in cells with all wild-type H2As, and we use $N_{H 2 A}=3$ in cells with only $1 / 2$ wild-type H2As. $N_{H 2 A} P_{p h o s}$ is the average number of $\gamma-\mathrm{H} 2 \mathrm{AXs}$ on a DNA fragment. The probability that the DNA fragment is pulled down is $P_{\text {pull-down }}=1-(1-f)^{N_{H A} P_{p h o s}}$ because this is the probability that at least one $\gamma-\mathrm{H} 2 \mathrm{AX}$ is 
successfully bound by an antibody (i.e. it is 1 minus the probability that none of the $\gamma$-H2AXs are bound by antibodies). This expression is not valid for $N_{H 2 A} P_{p h o s}<1$ (i.e. if the average number of $\gamma$-H2AXs on a DNA fragment is less than 1). If $N_{H 2 A} P_{p h o s}<1$, then we use the expression $P_{\text {pull-down }}=N_{H 2 A} P_{\text {phos }} f$ because $N_{H 2 A} P_{\text {phos }}$ is the probability of one $\gamma$-H2AX being present on the DNA fragment, and $f$ is the probability that the DNA fragment is pulled down given that there is one $\gamma$-H2AX on the fragment. Therefore, our quantitative model of the ChIP signal is the following:

$$
S\left(P_{\text {phos }}\right)= \begin{cases}C\left(1-(1-f)^{N_{H 2 A} P_{\text {phos }}}\right), & \text { when } N_{H 2 A} P_{\text {phos }} \geq 1 \\ C N_{H 2 A} P_{\text {phos }} f, & \text { when } N_{H 2 A} P_{\text {phos }}<1\end{cases}
$$

where $N_{H 2 A}, f$ and $C$ are parameters of the quantitative ChIP model, and $P_{\text {phos }}$ includes both the predicted DSB-dependent phosphorylation and the basal phosphorylation that occurs prior to DSB formation (see the next section on basal $\gamma$-H2AX levels). Equations (25) are applicable to all models besides the directed sliding model, which is discussed below in A quantitative model of ChIP for the directed sliding model.

As an example of the predictions from this model of ChIP, suppose it were the case that all the phosphorylatable H2As at a locus were phosphorylated - i.e. $P_{p h o s}(x, t)=1$. We use $f=0.16$ and $N_{H 2 A}=6$ for the wild type and $N_{H 2 A}=3$ for the mutant strain, which are the best parameter values extracted from Bayesian parameter estimation (as shown in Table 2). Then although the mutant strain has only $1 / 2$ as many $\gamma-\mathrm{H} 2 \mathrm{AXs}$ as the wild type, the mutant strain will have a ChIP signal that is 0.63 times the signal of the wild-type strain.

In the looping and 3D diffusion models, phosphorylation of one $\mathrm{H} 2 \mathrm{~A}$ is independent of phosphorylation of another H2A, even in the case of H2As on the same DNA fragment. For the looping model, this is because phosphorylation occurs according to the thermodynamic equilibrium looping probabilities, so the phosphorylation of one $\mathrm{H} 2 \mathrm{~A}$ has no influence on whether a nearby $\mathrm{H} 2 \mathrm{~A}$ is phosphorylated. For the 3D diffusion model, a single copy of a kinase is unlikely to contact more than one $\mathrm{H} 2 \mathrm{~A}$ on a given DNA fragment since the probability of the kinase contacting an $\mathrm{H} 2 \mathrm{~A}$ is at most 0.1 (this is estimated by plugging in reasonable values for the 
coefficients in the formula for $P_{\text {contact }}(x, l)$ in the Supplementary Information, 3D Diffusion Model Derivation); therefore, the phosphorylation of $\mathrm{H} 2 \mathrm{As}$ is effectively uncorrelated. The independence of $\mathrm{H} 2 \mathrm{~A}$ phosphorylation means that the number of $\gamma$-H2AXs on a DNA fragment is binomially distributed with a peak at $N_{H 2 A} P_{p h o s}$. In the looping and 3D diffusion models, for simplicity we ignore the spread of the binomial distribution and just use $N_{H 2 A} P_{p h o s}$ as the number of $\gamma-\mathrm{H} 2 \mathrm{AXs}$ on a DNA fragment. This simplifying assumption is what we included in equations (25).

In the 1D diffusion model, however, phosphorylation of one $\mathrm{H} 2 \mathrm{~A}$ is not independent of another H2A on the same DNA fragment, and so it is possible that there would be a wide range in the number of $\gamma-\mathrm{H} 2 \mathrm{AXs}$ on a DNA fragment, and so using just the mean number may not be accurate. Therefore, in every run of the 1D diffusion simulation, we record the number of $\gamma$-H2AXs on each DNA fragment. We apply the ChIP model to every DNA fragment and then take the average over the fragments from all runs in order to get the net ChIP signal.

\section{Basal $\gamma-\mathrm{H} 2 \mathrm{AX}$ levels are incorporated into the predicted ChIP signals}

Experimentally we measure a basal level of $\gamma-\mathrm{H} 2 \mathrm{AX}$ on the chromosome that exists at time 0 (which is before the DSB forms) - this is the ChIP signal measured at time 0 in Figures 1, 2, 3, 5 , 6 and 7. We use the mean values of the basal $\gamma$-H2AX profile to serve as the initial ChIP signal in every model of $\gamma$-H2AX spreading (rather than starting from a ChIP signal of 0). Because basal phosphorylation and DSB-dependent phosphorylation are presumably independent of each other, the probability of phosphorylation as a result of either basal or DSB-dependent phosphorylation is

$$
P_{\text {phos }, \text { total }}(x, t)=P_{\text {phos, basal }}(x)+\left(1-P_{\text {phos, basal }}(x)\right) P_{\text {phos }}(x, t)
$$

where $P_{\text {phos }}(x, t)$ is the DSB-dependent phosphorylation derived for the model of $\gamma-\mathrm{H} 2 \mathrm{AX}$ spreading. To obtain $P_{\text {phos, basal }}$, we note that the basal ChIP signals are small, so $P_{\text {phos, basal }}$ must be small. Therefore, we can use the bottom equation (25), and rearrange to get $P_{\text {phos, basal }}(x)=\frac{S_{\text {exp basal }}(x)}{C N_{H 2 A} f}$, where $S_{\text {exp basal }}(x)$ is the experimentally measured basal ChIP 
signal. To obtain the predicted ChIP signals, $P_{p h o s, t o t a l}(x, t)$ is what we actually use in equations (25) in place of $P_{\text {phos }}$.

\section{A quantitative model of ChIP for the directed sliding model}

For the directed sliding model, we modify equations (25). In the directed sliding model we assume that whenever a kinase slides to a locus, it phosphorylates all H2As there. However, the kinase does not make it all the way to every locus. In the directed sliding model, $P_{p h o s}(x, t)$ is equivalent to the probability that the kinase arrives to location $x$. If the kinase does not arrive to the location $x$, then the ChIP signal is the basal ChIP signal $S_{\text {exp basal }}(x)$, but if the kinase does arrive to the location, then the ChIP signal is $C\left(1-(1-f)^{N_{H 2 A}}\right)$. Therefore, the ChIP signal for the directed sliding model is given by

$$
S\left(P_{\text {phos }}\right)=S_{\text {exp basal }}(x)\left(1-P_{\text {phos }}(x, t)\right)+P_{\text {phos }}(x, t) C\left(1-(1-f)^{N_{H 2 A}}\right)
$$

\section{Bayes factor calculation}

The Bayes factor is given by the ratio of marginal likelihoods for two models, Models A and B:

$$
\text { Bayes Factor }=\frac{\int P\left(\vec{y} \mid \vec{\theta}_{A}, \text { Model A) } P\left(\vec{\theta}_{A}\right) d \vec{\theta}_{A}\right.}{\int P\left(\vec{y} \mid \vec{\theta}_{B}, \text { Model B) } P\left(\vec{\theta}_{B}\right) d \vec{\theta}_{B}\right.}
$$

where $P\left(\vec{y} \mid \vec{\theta}_{A}\right.$, Model A) is the likelihood of the experimental data $\vec{y}$ given Model A and the model parameters $\vec{\theta}_{A}$, and $P\left(\vec{\theta}_{A}\right)$ is the prior probability of $\vec{\theta}_{A}$ (likewise for Model B). These are discussed below. In the tables displaying the Bayes factors (Table 1 and S1), Model B is always the model with the highest marginal likelihood compared to the other models in the table.

We use previous studies to estimate the range of possible parameter values - the integral in equation (28) is over this range. The previous studies measured the chromatin Kuhn length (95\% $\mathrm{CI}$ is 8.4-15 kb) and linear density of chromatin DNA (95\% CI 56-68 bp/nm) (1), and density of the nucleosomes along the chromatin (about $170 \mathrm{bp} /$ nucleosome) (12) and provided data on the 
kinetics of in vivo H2A phosphorylation that allow us to constrain kinetic parameter ranges (12). Ref. (10) was used to constrain RE parameters. In some cases, there is some arbitrariness in the choice of the range, but choosing a different range (that is still reasonable given the previous studies) only changes the Bayes factors by an order of magnitude. The ChIP parameter ranges are as follows: $f$ is allowed to vary between 0 and $1, \mathrm{~N}$ is allowed to be 2,4 or $6 \mathrm{H} 2 \mathrm{As}$, and $C$ is allowed to vary between 8.3 and 280, which is based on our measurements of DNA recovery from ChIP compared to samples in which PCR was performed without ChIP.

The functional form of the prior probability distribution $P(\theta)$ for each parameter 6 is as follows: For the parameters whose values we know within an order of magnitude (i.e. the Kuhn length $l$ and ChIP parameter $N_{H 2 A}$ ), we use the uniform prior $P(\theta)=$ constant. For parameters that are less certain, we use Jeffreys priors. However, it is difficult to obtain exact Jeffreys priors given the complexity of our models' functional forms, so we use Jeffreys priors from similar but simpler scenarios. For parameters that represent the probability of a binary event (i.e. the ChIP parameter $f$, the directed sliding model's parameter $q$, and the RE-MAT binding fraction $F_{R E}$ ), $P(\theta)=\frac{1}{\sqrt{\theta(1-\theta)}}(16)$. All other parameters are assigned prior distributions of $P(\theta)=\frac{1}{\theta}$, which is the Jeffreys prior for the rate parameter of an exponential distribution. All prior distributions are normalized and constrained to be within the estimated range of possible values (i.e. $P(\theta)=0$ if 6 is outside the range of possible values). For simplicity, we assume that $P(\vec{\theta})$ for the parameter set $\vec{\theta}$ is the product of the priors $\prod_{i} P\left(\theta_{i}\right)$ for the individual parameters $\theta_{i}$.

Because our priors are not precisely Jeffreys priors, let us estimate the extent to which this lack of precision could affect the Bayes factor. To do so, one can calculate the Bayes factor using a different prior. For example, consider using a uniform prior for all model parameters for both the directed sliding model and the 1D diffusion model. For most of the parameters, a uniform prior is quite different from the Jeffreys prior; the uniform prior will serve as an extreme example, so the Bayes factor will presumably be changed by more than it would be if we were able to use the exact Jeffreys prior. As shown in Table $1 B$ of the main text, the Bayes factor is $10^{-6}$ when Mec 1 is 
undergoing 3D diffusion and Tel1 is undergoing 1D diffusion (this Bayes factor is in comparison to Mec1 3D diffusion with Tel1 directed sliding). Using all uniform priors, this Bayes factor becomes $10^{-7}$. Therefore, using a prior with a very different functional form can change the Bayes factor by an order of magnitude but does not change our conclusions.

Finally, we discuss the likelihood function. Consider a particular theory, e.g. Model A. For a particular parameter set $\vec{\theta}$ and for the $i$ th measurement condition (each measurement condition is composed of the yeast strain, the time point and the location on the chromosome), Model A predicts a single $\gamma$-H2AX ChIP signal $S_{i}$. However, because there is measurement error in the experiments, our theory should predict that the experimental data will have some spread about the mean predicted ChIP signal. Therefore, we incorporate an error model into the theory, which we assume to be Gaussian error - i.e. model A predicts that the mean ChIP signal is $S_{i}$ and predicts a Gaussian spread about the mean with standard deviation $\sigma_{i}$. Hence the likelihood function is

$$
P\left(\vec{y} \mid \vec{\theta}_{A}, \text { Model A }\right)=\prod_{i, j}\left[\frac{1}{\sqrt{2 \pi} \sigma_{i}} \exp \left(\frac{-\left(S_{i}-y_{i j}\right)^{2}}{2 \sigma_{i}^{2}}\right)\right]
$$

The experimentally measured ChIP signal $y_{i j}$ has the subscript $i$ for the measurement condition and the subscript $j$ because there are multiple data collected for the same measurement condition.

Analysis of all of our ChIP data revealed that for each measurement condition, the standard deviation of the ChIP data is approximately equal to the mean ChIP signal multiplied by 0.35 . Therefore, our error model can use $\sigma_{i}=0.35 S_{i}$. Including this into equation (29) yields

$$
P\left(\vec{y} \mid \vec{\theta}_{A}, \text { Model A }\right)=\prod_{i, j}\left[\frac{1}{0.35 \sqrt{2 \pi} S_{i}} \exp \left(\frac{-\left(S_{i}-y_{i j}\right)^{2}}{2\left(0.35 S_{i}\right)^{2}}\right)\right]
$$

Finally, the integral in equation (28) is carried out by evaluating the integrand at points on a grid in parameter space and then using a Riemann sum.

As previously discussed, most theories have two variants: (1) a model in which there is an increasing phosphorylation rate or increasing rate of diffusion initiation, and (2) a model in which there is a constant phosphorylation rate or constant rate of diffusion initiation. For each theory, we 
use the best variant when reporting the Bayes factors. (We found that the constant phosphorylation rate/constant diffusion initiation rate models always fit the data better than the models with the increasing rates).

When calculating the Bayes factor for Tell and Mec1 models simultaneously, we exclude the Tel1 3D models because they were proven to be very unlikely from the Bayes factor analysis of Tel1 individually. This exclusion is justified because the analysis in Table $1 A$ indicates that the $1 \mathrm{D}$ models are extremely favored for Tell, and by inspecting the best ChIP parameter values for the various Tel1 models, we see that the 1D models would still be the best models for Tell even when constrained to have the same ChIP parameter values as Mec1. 


\section{Supplemental Figures}

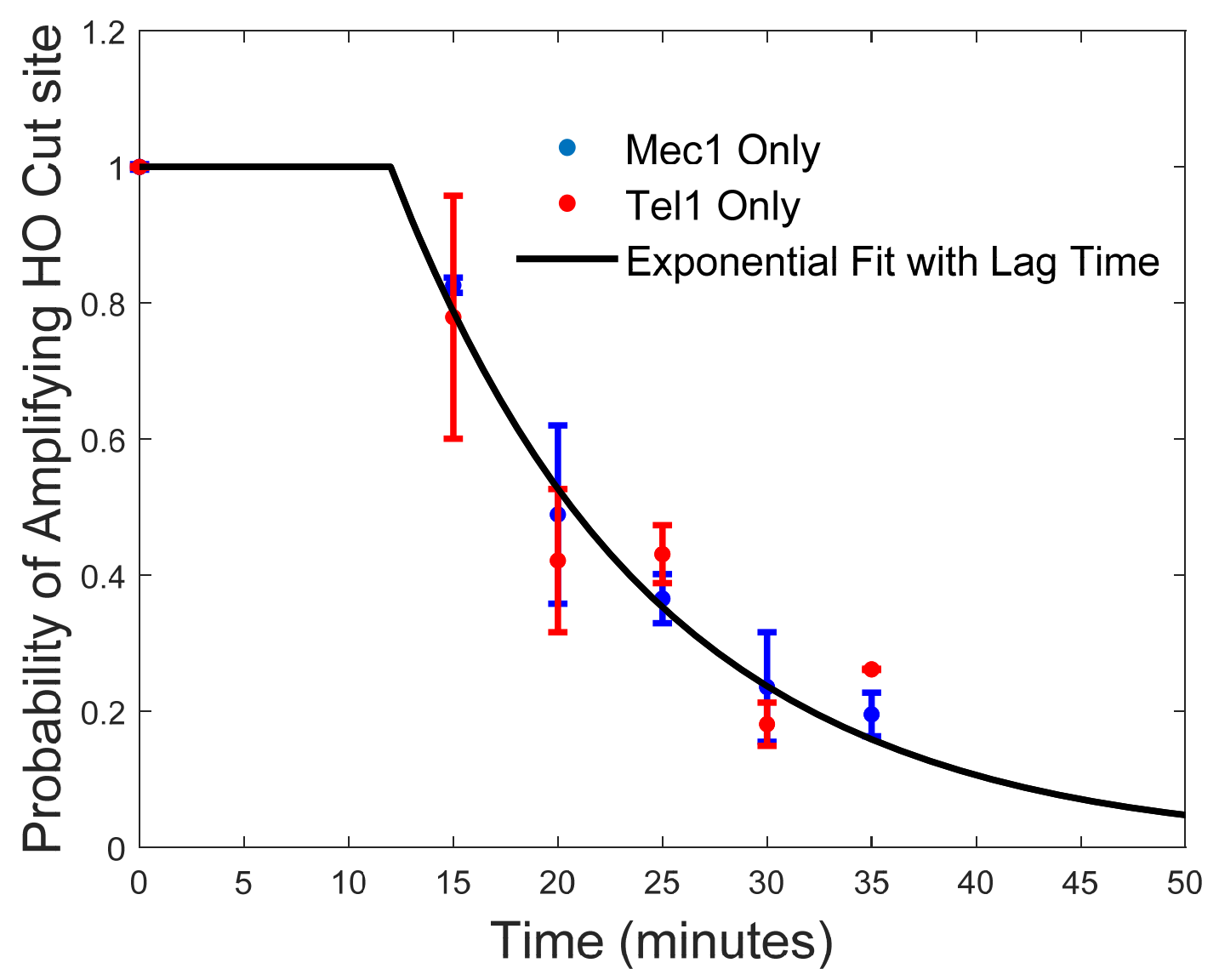

Figure S1: Kinetics of DSB induction

The amount of cleavage at the MAT locus by HO endonuclease after galactose induction was monitored in strains deleted for YKU80 and carrying either Mec1 (blue) or Tel1 (red). qPCR was performed with primers flanking the cut site to quantify the levels of cleavage over 35 mins. In the presence of a DSB, qPCR fails to amplify the DNA. Approximately, $80 \%$ cutting was achieved by 30 mins. The black line is an exponential fit to both strains, using a lag time of 12 minutes (reflecting transcription and translation of the $\mathrm{HO}$ endonuclease) and a DNA cleavage rate of $k_{D S B}$ $=0.08 /$ minute. The lag time and $k_{D S B}$ are used in calculating our models of $\gamma-\mathrm{H} 2 \mathrm{AX}$ spreading. Error bars represent the standard error of the mean from $n \geq 3$ measurements. 


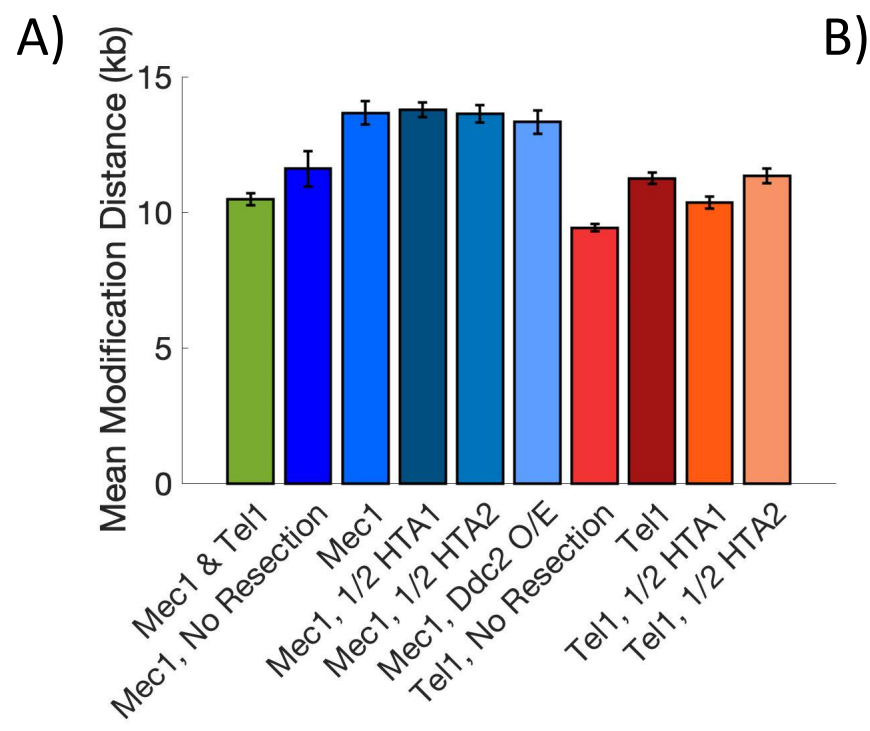

B)

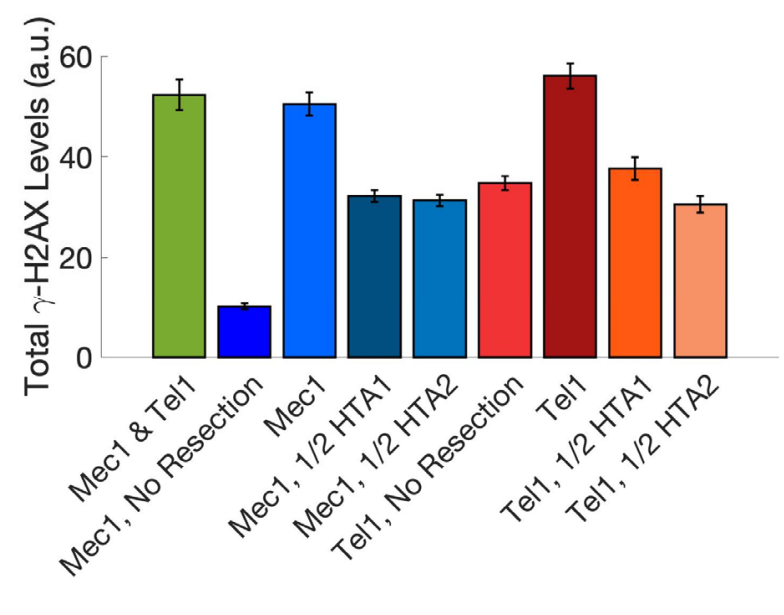

Figure S2: Mean modification distance and total $\gamma-\mathrm{H} 2 \mathrm{AX}$ accumulation

A) The mean modification distance (MMD), the distance from the break that encompasses half the $\gamma$-H2AX profile, is displayed for all strains at 75 min except for Mec1, Ddc2 O/E which was measured at $60 \mathrm{~min}$. Error bars represent the standard error of the mean. B) Total $\gamma$-H2AX levels were calculated by summing up $\gamma-\mathrm{H} 2 \mathrm{AX}$ levels across all measured distances at $75 \mathrm{~min}$. Error bars represent the standard error of the mean. 


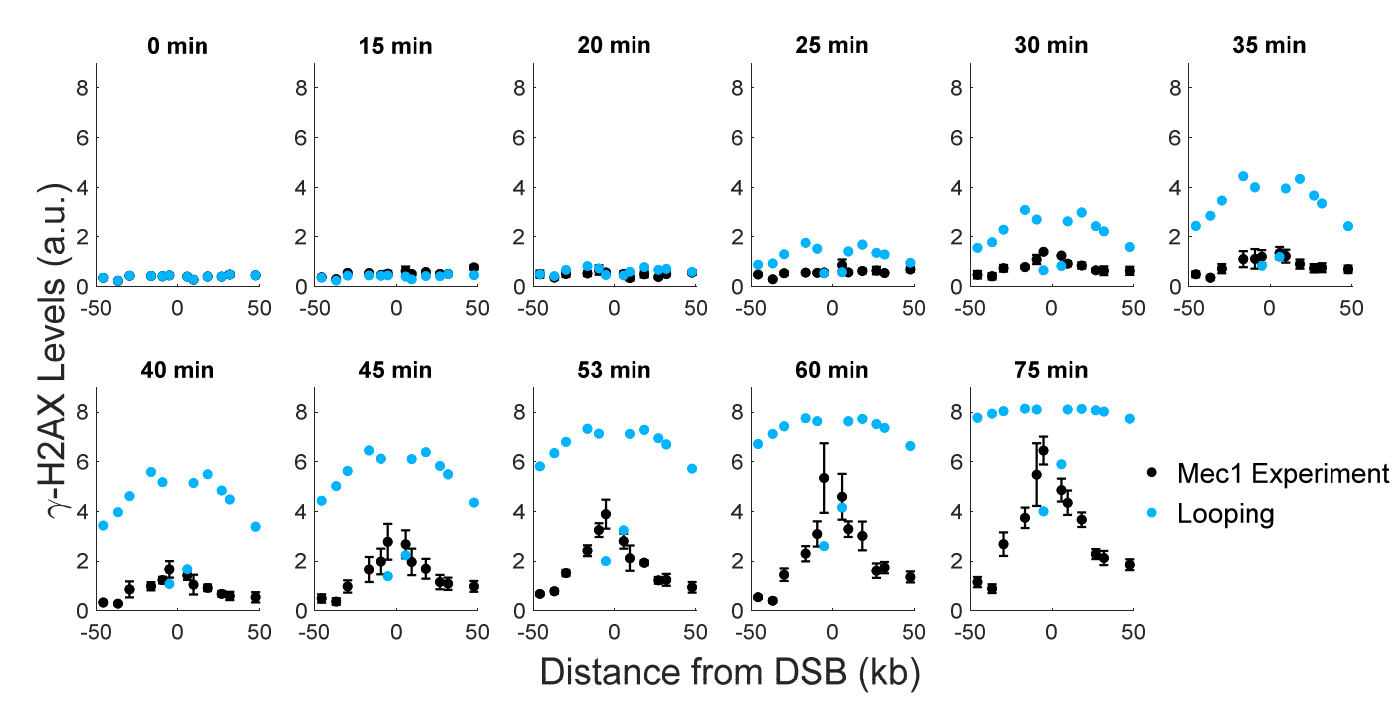

Figure S3: Comparison of experimental data by Mec1 to a looping model with a linearly increasing phosphorylation rate. Comparisons of the predicted phosphorylation levels to experimentally measured $\gamma$-H2AX levels. This model fits the data much worse than the best model, which is shown in Figure 5. Experimental error bars are the standard error of the mean from $n \geq 3$ measurements. Bayesian parameter estimation was used to simultaneously fit the model to the data shown here and to the RE-adjacent measurements from Figure 3. The optimal parameter values from Bayesian parameter estimation were used to plot the model, and are as follows: $C=44, f=$ $0.035, N_{H 2 A}=6, l=8.4 \mathrm{~kb}$, and $\psi=0.039 /$ minute $^{2}$. In Figure 5 and Table $1 \mathrm{~B}, \mathrm{Mec} 1$ and Tel1 are constrained to the same values $N_{H 2 A}, f$ and $C$ for the ChIP parameters. 


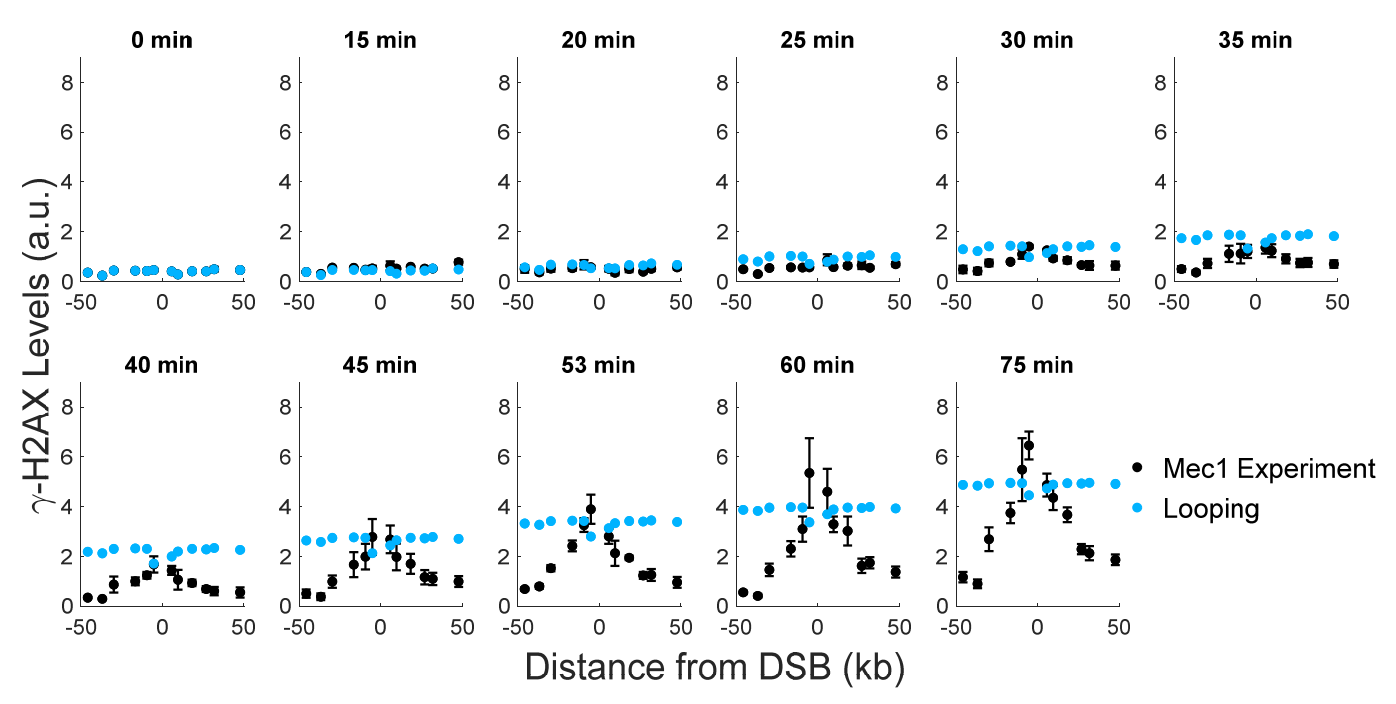

Figure S4: Comparison of experimental data by Mec1 to a looping model with a constant phosphorylation rate. The best model parameters are $C=120, f=0.035, N_{H 2 A}=2, l=8.4 \mathrm{~kb}, \varphi$ $=11 /$ minute, and $k_{\text {init }}=0.017 /$ minute. For further details, refer to the caption of Figure S3. 


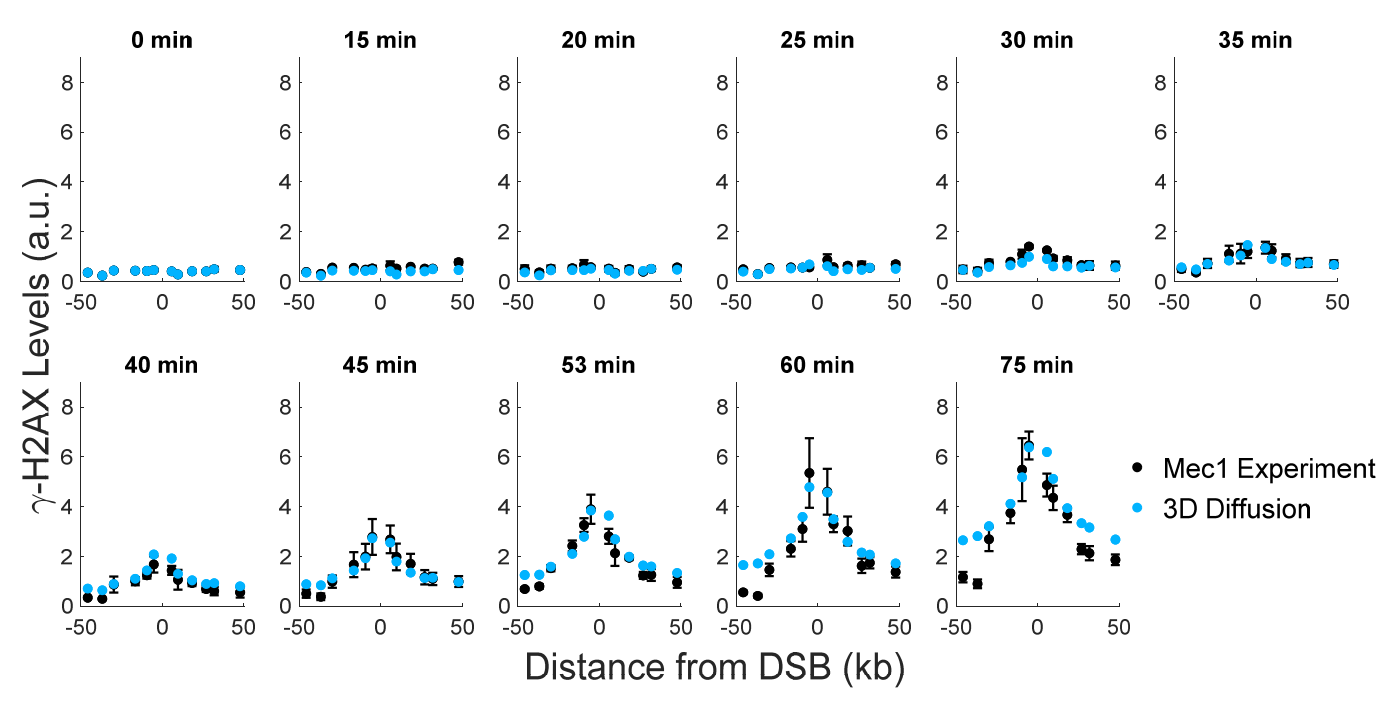

Figure S5: Comparison of experimental data by Mec1 to a 3D diffusion model with a linearly increasing rate of diffusion initiation. The best model parameters are $C=13, f=0.16, N_{H 2 A}=6$, $l=15 \mathrm{~kb}$, and $\zeta=3.7 \times 10^{-4} /$ minute $^{2}$. For further details, refer to the caption of Figure S3. 


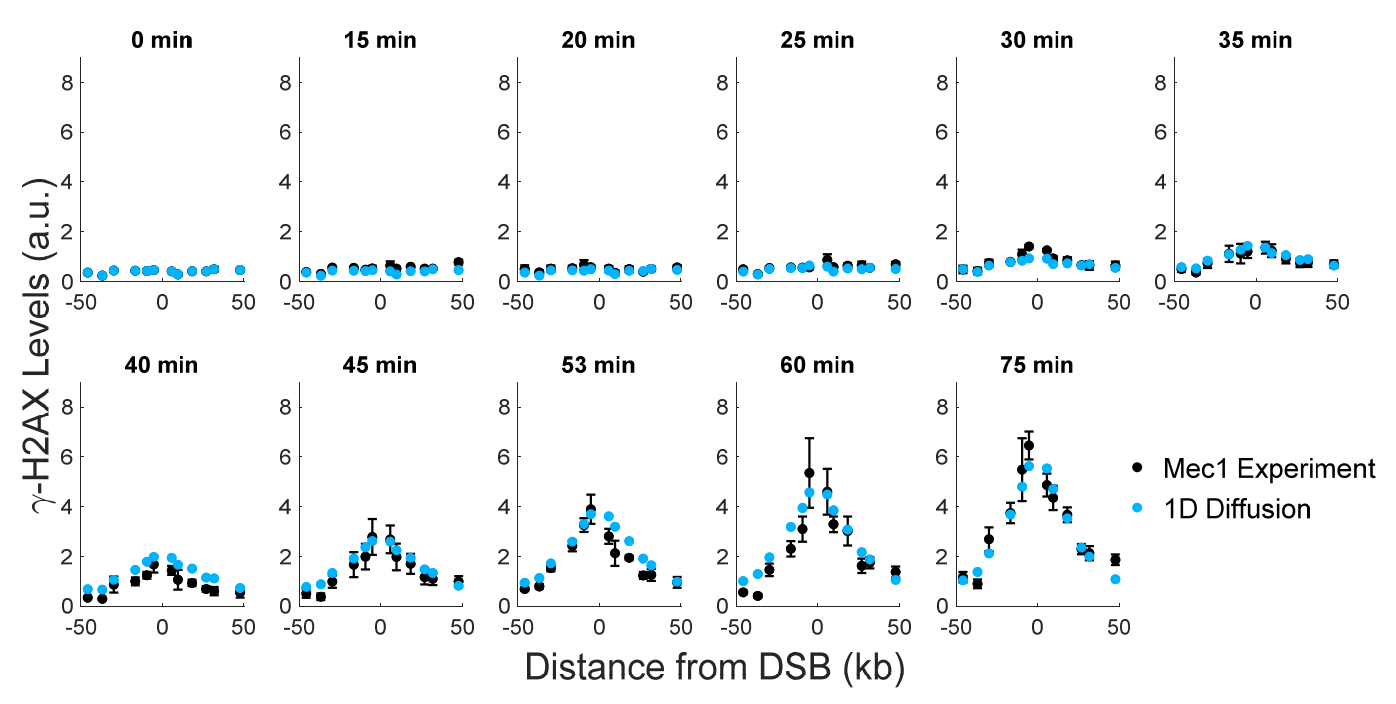

Figure S6: Comparison of experimental data by Mec1 to a 1D diffusion model with a linearly increasing rate of diffusion initiation. The best model parameters are $C=11, f=0.80, N_{H 2 A}=6$, $D=18 \mathrm{~kb}^{2} /$ minute, $k_{\text {cat }}=0.039 /$ minute, and $z=84 /$ minute $^{2}$. For further details, refer to the caption of Figure S3. 


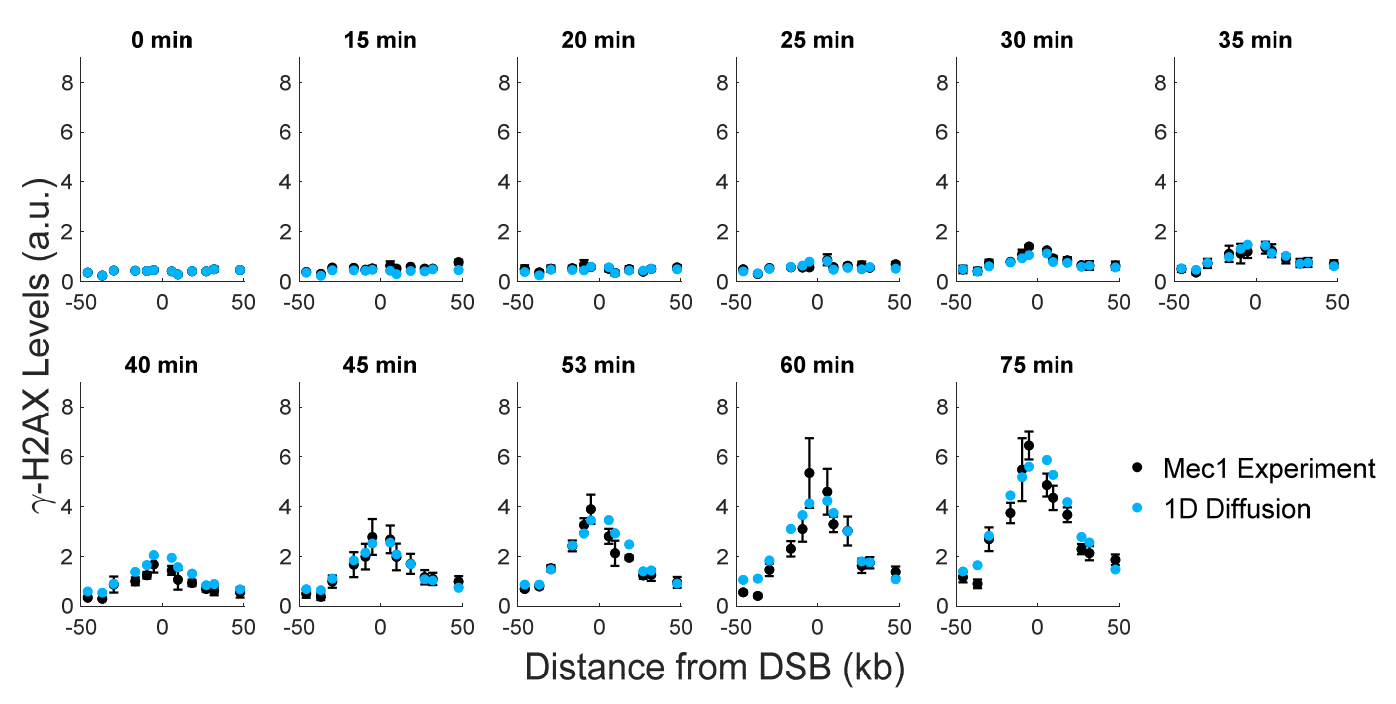

Figure S7: Comparison of experimental data by Mec1 to a 1D diffusion model with a constant rate of diffusion initiation. The best model parameters are $C=32, f=0.28, N_{H 2 A}=6, D=22$ $\mathrm{kb}^{2} /$ minute, $k_{\text {cat }}=1.9 /$ minute, and $k_{\text {init }}=0.019 /$ minute. For further details, refer to the caption of Figure S3. 


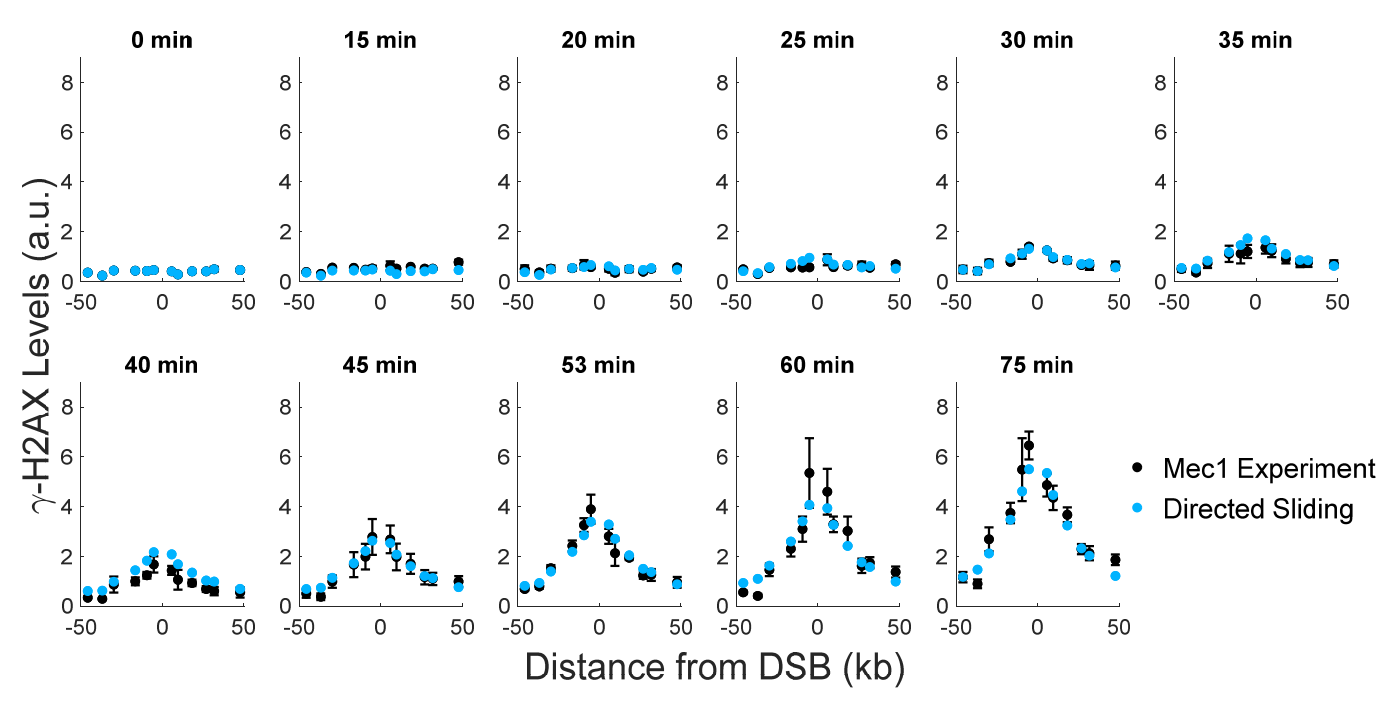

Figure S8: Comparison of experimental by Mec1 to a directed sliding model. The best model parameters are $C=73, f=0.72, N_{H 2 A}=2, k_{\text {slide }}=17 \mathrm{~kb} /$ minute, $k_{\text {off }}=0.74 /$ minute, and $k_{\text {init }}=$ $0.0020 /$ minute. For further details, refer to the caption of Figure S3. 


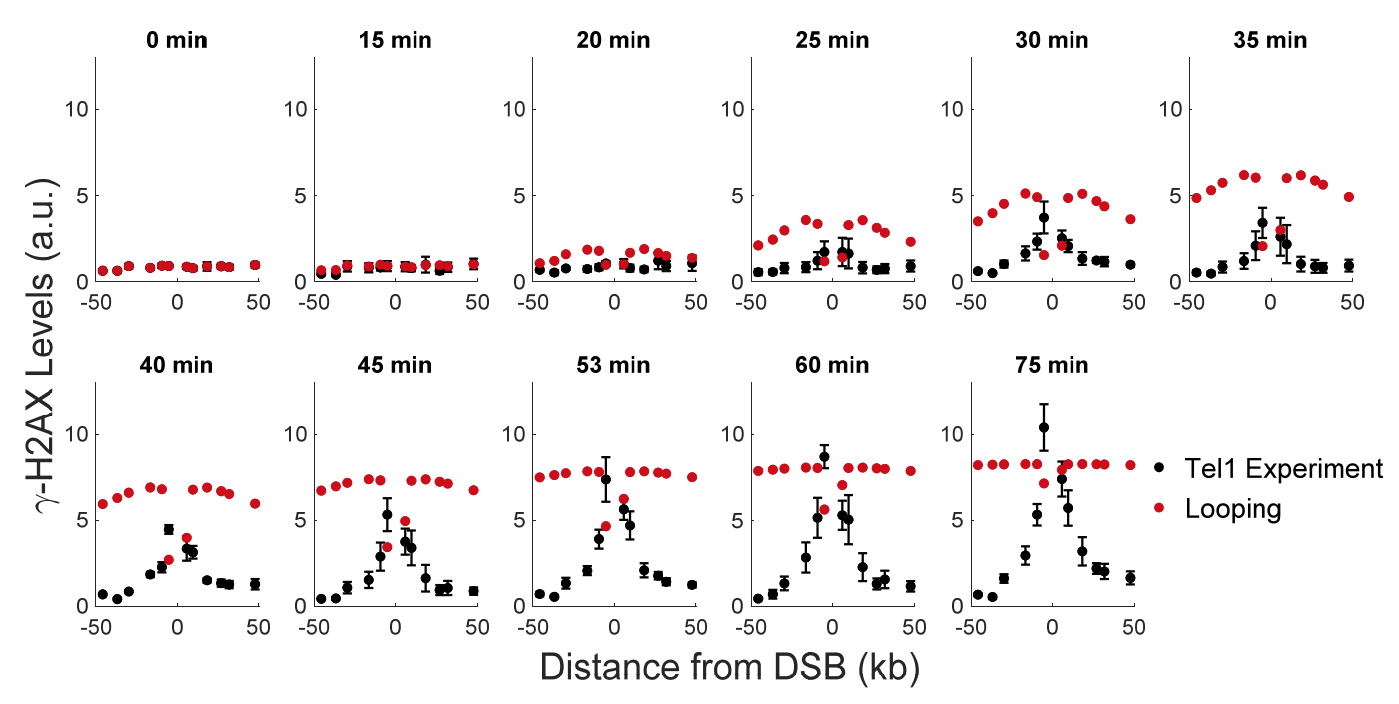

Figure S9: Comparison of experimental data by Tel1 to a looping model with a linearly increasing phosphorylation rate. The best model parameters are $C=120, f=0.035, N_{H 2 A}=2, l$ $=8.4 \mathrm{~kb}$, and $\psi=0.14 /$ minute $^{2}$. For further details, refer to the caption of Figure S3. 


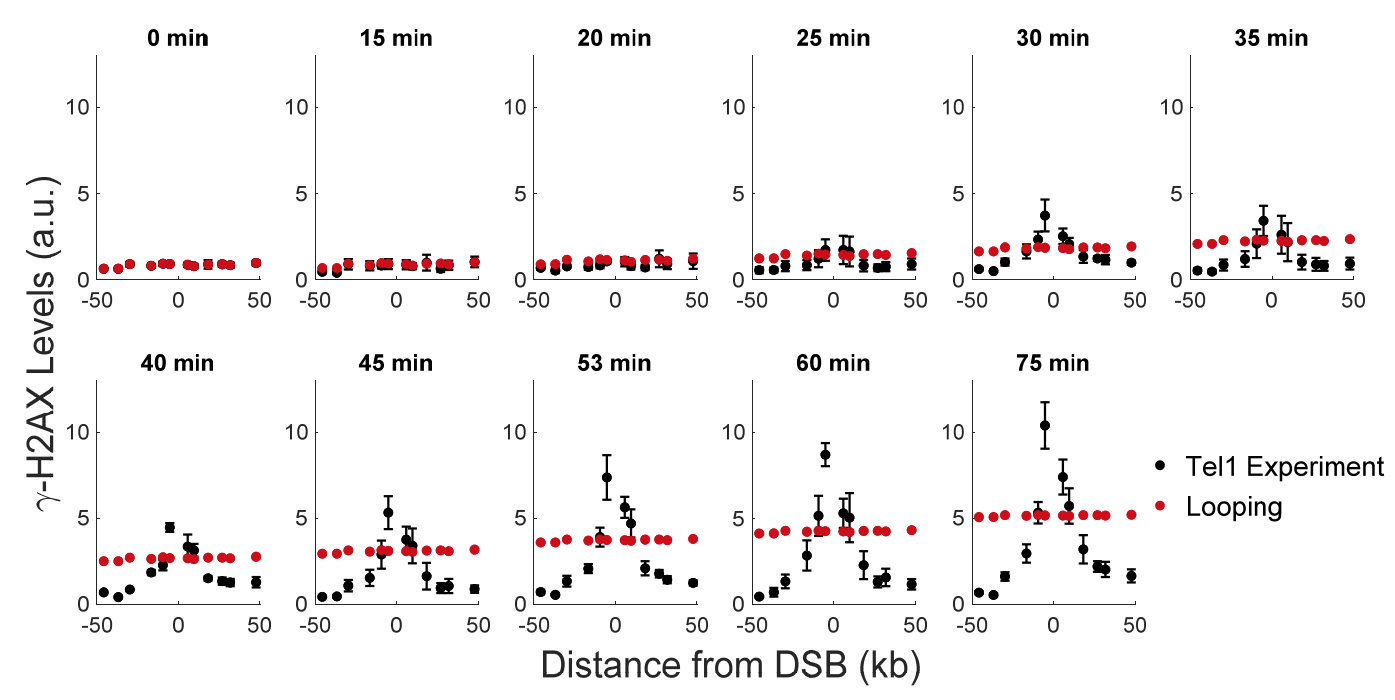

Figure S10: Comparison of experimental data by Tel1 to a looping model with a constant phosphorylation rate. The best model parameters are $C=120, f=0.035, N_{H 2 A}=2, l=8.4 \mathrm{~kb}, \varphi$ $=200 /$ minute, and $k_{\text {init }}=0.017 /$ minute. For further details, refer to the caption of Figure S3. 


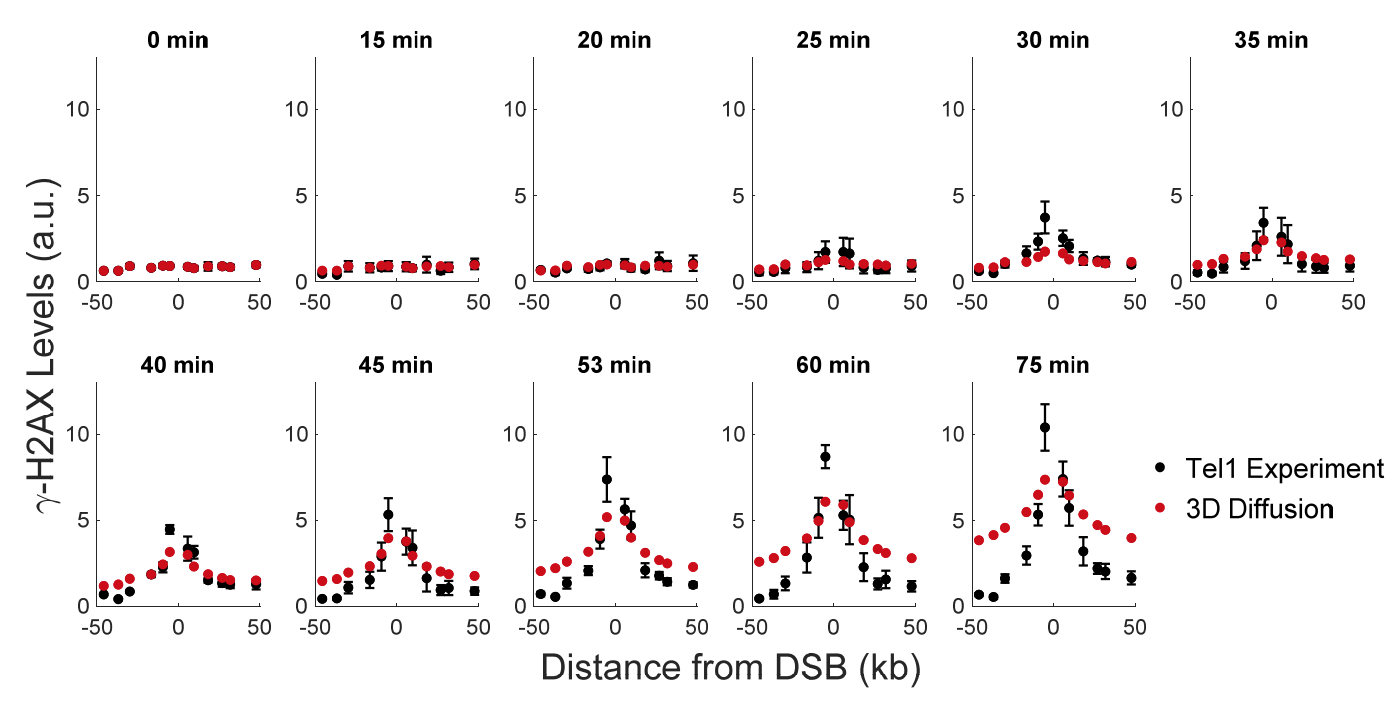

Figure S11: Comparison of experimental by Tel1 to a 3D diffusion model with a linearly increasing rate of diffusion initiation. The best model parameters are $C=13, f=0.16, N_{H 2 A}=6$, $l=15 \mathrm{~kb}$, and $\zeta=6.2 \times 10^{-4} /$ minute $^{2}$. For further details, refer to the caption of Figure S3. 


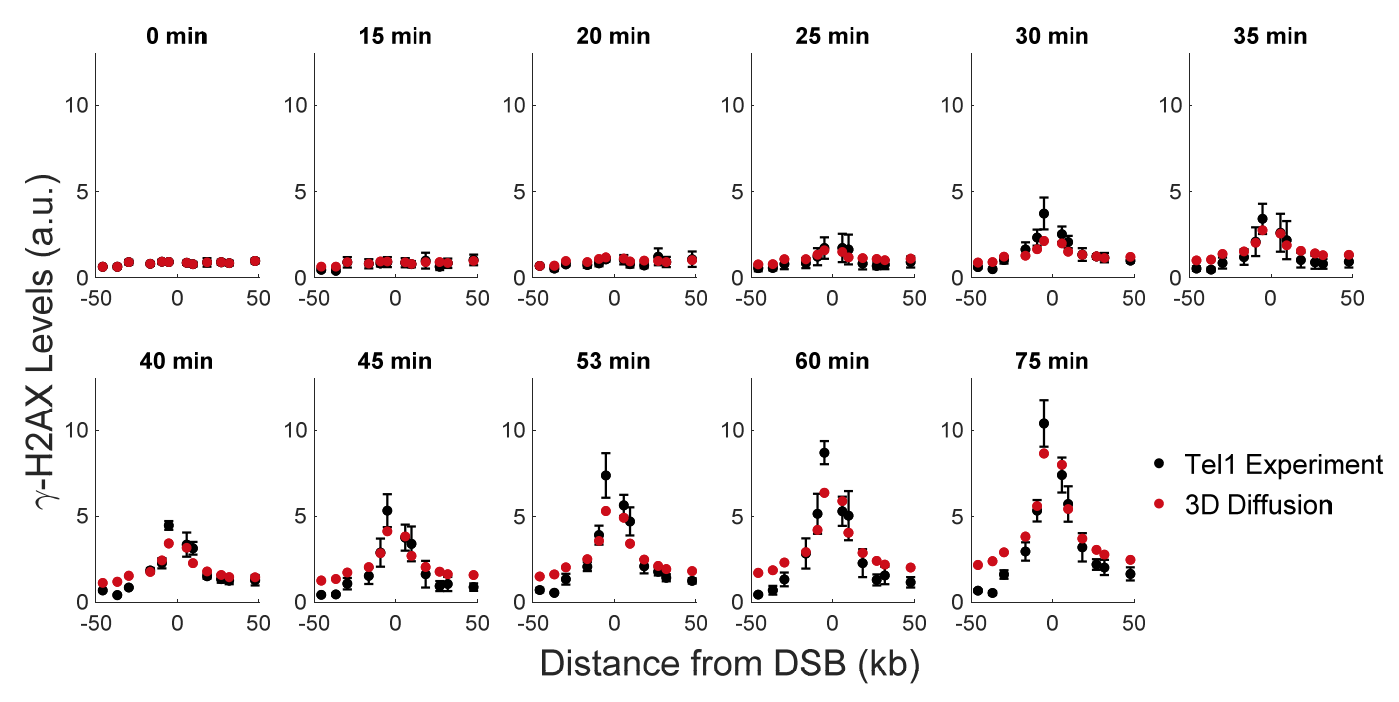

Figure S12: Comparison of experimental data by Tel1 to a 3D diffusion model with a constant rate of diffusion initiation. The best model parameters are $C=73, f=0.55, N_{H 2 A}=6, l$ $=15 \mathrm{~kb}, \omega=0.00030 /$ minute, and $k_{\text {init }}=1.0 /$ minute. For further details, refer to the caption of Figure S3. 


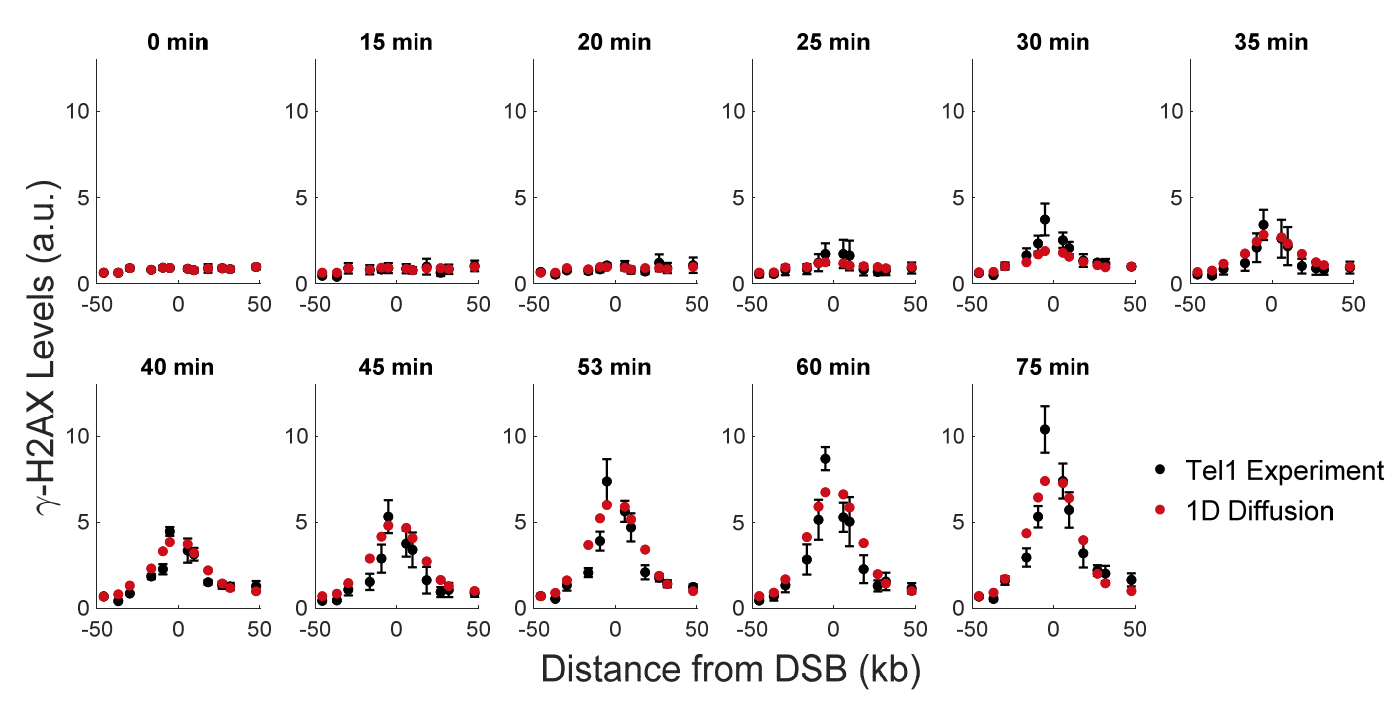

Figure S13: Comparison of experimental data by Tel1 to a 1D diffusion model with a linearly increasing rate of diffusion initiation. The best model parameters are $C=9.3, f=0.50, N_{H 2 A}=$ $4, D=3.9 \mathrm{~kb}^{2} /$ minute, $k_{c a t}=0.59 /$ minute, and $z=37 /$ minute $^{2}$. For further details, refer to the caption of Figure S3. 


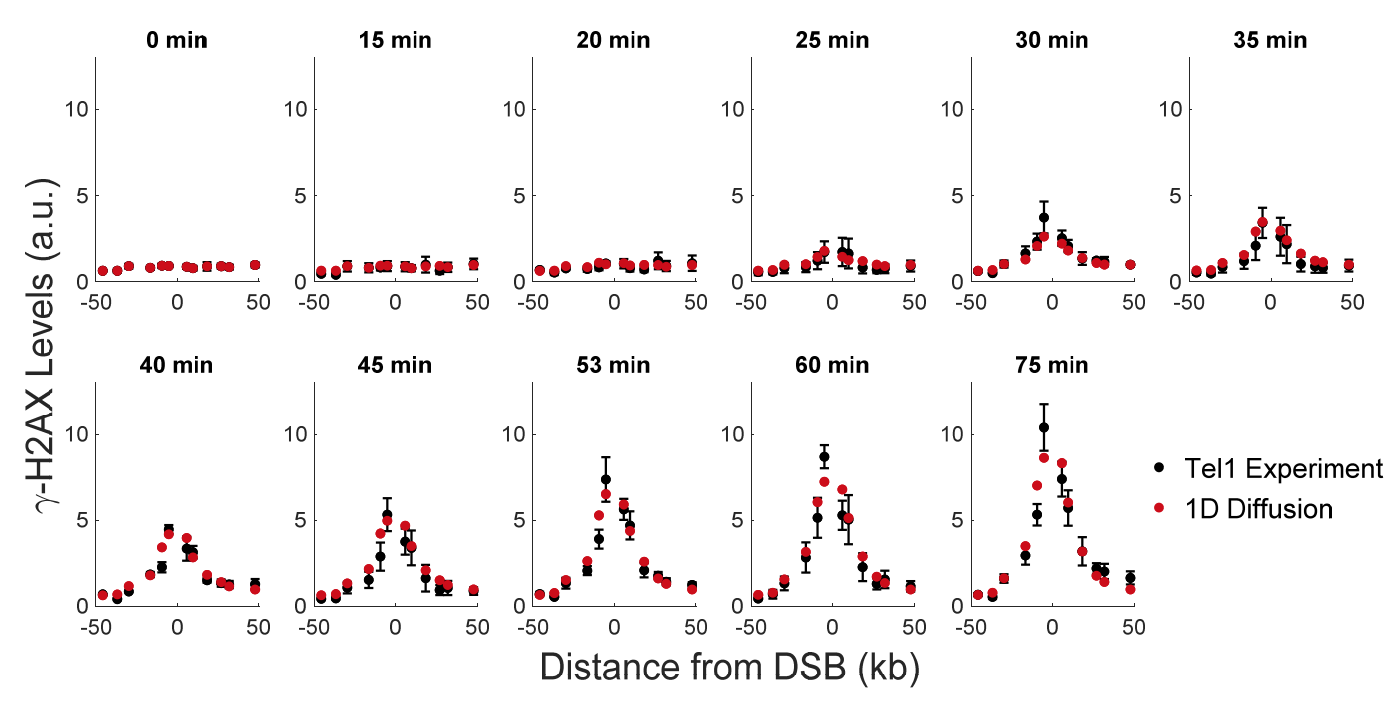

Figure S14: Comparison of experimental data by Tel1 to a 1D diffusion model with a constant rate of diffusion initiation. The best model parameters are $C=32, f=0.55, N_{H 2 A}=4$, $D=4.1 \mathrm{~kb}^{2} /$ minute, $k_{\text {cat }}=0.20 /$ minute, and $k_{\text {init }}=0.098 /$ minute. For further details, refer to the caption of Figure S3. 


\section{Supplemental Tables}

\section{Table S1: $\log _{10}$ (Bayes Factor) for all mechanisms}

The $\log _{10}$ (Bayes Factor) is shown for every model variant. (Table 1 showed the Bayes factors for models with a constant rate of diffusion initiation or constant phosphorylation rate). The Bayes factor is calculated by dividing the probability of the indicated model by the probability of the best model. Bayes factors were computed for Mec1 and Tell separately. For Mec1, the best model is 3D diffusion with a constant rate of diffusion initiation. For Tel1, the best model is directed sliding.

$$
\text { Model Mec1, } \left.\log _{10} \text { (Bayes Factor }\right) \quad \text { Tel1, } \log _{10}(\text { Bayes Factor })
$$

Looping with Linearly

Increasing Phosphorylation

Rate

Looping with Constant

Phosphorylation Rate

3D Diffusion with Linearly

Increasing Rate of Diffusion

Initiation

3D Diffusion with Constant

Rate of Diffusion Initiation

Directed Sliding

1D Diffusion with Linearly

Increasing Rate of Diffusion

Initiation

1D Diffusion with Constant

Rate of Diffusion Initiation
$-545$

$-252$

$-28$

$-136$

Best Model $-61$

$-14$

Best Model

$-28$

$-35$

$-12$ 
Table S2: List of yeast strains used in this study

\begin{tabular}{|c|c|}
\hline Strain & Genotype \\
\hline JKM139 & $\begin{array}{l}\text { MATa } h m l \Delta:: A D E 1 \text { hmr } \triangle:: A D E 1 \text { adel-100 leu2-3,112 lys5 trpl::his } G^{\prime} \text { ura3-52 } \\
\text { ade3::GAL::HO }\end{array}$ \\
\hline yFD508 & JKM139 tel1 $\Delta:: T R P 1$ barl $\triangle:: A D E 3$ \\
\hline yFD538 & JKM139 mec1 $1:: N A T \operatorname{sml} 1 \Delta:: K A N$ barl $\triangle:: A D E 3$ \\
\hline yKL002 & JKM139 tel1 $\Delta:: T R P 1$ barl $\Delta:: A D E 3$ nej $1 \Delta:: H P H$ \\
\hline yKL003 & JKM139 mec1 $1:: N A T$ sml1 $\triangle:: K A N$ barl $\triangle:: A D E 3$ nej1 $1:: H P H$ \\
\hline yKL004 & 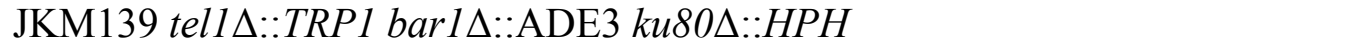 \\
\hline yKL005 & 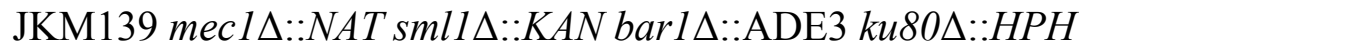 \\
\hline yKL018 & 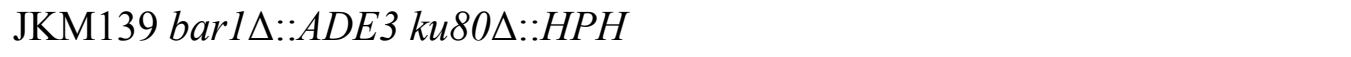 \\
\hline yKL019 & yKL004 GAL1-Ddc2 (plasmid PML105.45 integrated at leu2-3,112) \\
\hline yKL025 & yKL004 htal-S129A \\
\hline yKL026 & yKL004 hta2-S129A \\
\hline yKL040 & yKL005 hta2-S129A \\
\hline yKL041 & yKL005 htal-S129A \\
\hline
\end{tabular}


Table S3: Primers used during strain construction

\begin{tabular}{|c|c|}
\hline Primer & Sequence \\
\hline BL325 & CTTCTCAAGAATTATAAGATGTTTT \\
\hline BL326 & ATCTTATAATTCTTGAGAAGGATCA \\
\hline BL327 & $\begin{array}{l}\text { GAAGTCTGCCAAGGCTACCAAGGCTGCCCAAGAATTATAAGATC } \\
\text { GGTTCTGGTATTTTAAAGAAGGCGGAAGGAACTAAA }\end{array}$ \\
\hline BL329 & TTACAGTTCTTGAGAAGCTTGTTTT \\
\hline BL330 & AAGCTTCTCAAGAACTGTAAGATCA \\
\hline BL331 & $\begin{array}{l}\text { CAAAACTTGTTGCCAAAGAAGTCTGCCAAGACTGCCAAAGCTGCC } \\
\text { CAAGAACTGTAAGAACTGAGTTGAAAAGAAACAAA }\end{array}$ \\
\hline HPHorf +751 & CAGAGCTTGGTTGACGGC \\
\hline KL020 & CAGCCAGTGGATCGTAAATA \\
\hline KL021 & ACAGTGCCCAATGAACCTAA \\
\hline KL022 & TCCGGTGGTAAAGGTGGTAA \\
\hline KL023 & ATTAACCTGGGGGCCATAAA \\
\hline KL024 & CAAAAGAAAGAGAGCCTAGCTG \\
\hline ku80ORFL & CTCTTGAGTGTCTTTACCGC \\
\hline KU80T2 & CTAGTTCAGCAACCGAAATCC \\
\hline ku80MX18 & $\begin{array}{l}\text { TAACGAGAGTGCAGGACATATGCACAAATAATATATCTCACACCA } \\
\text { TAATAGCATAGGCCACTAGTGGATCTG }\end{array}$ \\
\hline ku80MX19 & $\begin{array}{l}\text { CTCTTTAACTGTGGTGACGAAAACATAACTCAAAGGATGTTAGAC } \\
\text { CTTTTCAGCTGAAGCTTCGTACGC }\end{array}$ \\
\hline nej1-225 & TCCAAAGACCTTTGGTCC \\
\hline Nej1-MXp1 & $\begin{array}{l}\text { AGAATAACCATAACAACGGCCAGATAGGAGGTTAATCTTACACA } \\
\text { TGTGTGGATATCAAGCTTGCCTCGTCCCCGC }\end{array}$ \\
\hline Nej1-MXp2 & $\begin{array}{l}\text { GAACTATTTGAAAGGTCCAACCTTAATTTTTGACGTTTAATTGACT } \\
\text { TGCCGTCGACACTGGATGGCGGCGTTAGTATC }\end{array}$ \\
\hline nej1+267 & TTTACAGTGATGGCGAGC \\
\hline
\end{tabular}


bioRxiv preprint doi: https://doi.org/10.1101/2019.12.17.877266; this version posted December 17, 2019. The copyright holder for this preprint (which was not certified by peer review) is the author/funder, who has granted bioRxiv a license to display the preprint in perpetuity. It is made available under aCC-BY-NC-ND 4.0 International license.

Table S4: Plasmids used during strain construction

Plasmid

Genotype

\begin{tabular}{ll}
\hline pAG32 & ampr, $H P H$ \\
pBL13 & ampr, Cas9, URA3, gRNA targeting HTA1 \\
pBL14 & ampr, Cas9, URA3, gRNA targeting HTA2 \\
pJH2972 & ampr, Cas 9, URA3 \\
PML105.45 & GAL1-Ddc2, LEU2 \\
\hline
\end{tabular}


Table S5: Primers used in quantitative PCR (qPCR)

\begin{tabular}{|c|c|}
\hline Primer & Sequence \\
\hline CTR86p1 & CGGTCCTCGATTTTGTTACCTTC \\
\hline CTR86p2 & GCAAGGATATTCCTGCCTTTTTC \\
\hline ERS1p1 & ACACCCTGAATGGGGAAAC \\
\hline ERS1p2 & CTGCATGGGTGCTTGATG \\
\hline HOCSp4 & TCGTCAACCACTCTACAAAACCA \\
\hline IMG1p1 & TGGATCATGGACAAGGTCCTAC \\
\hline IMG1p2 & GGCGAAAACAATGGCACTCT \\
\hline KL032 & TGATGGTCATGGTGGTAGCG \\
\hline KL033 & CTTTGCGTCCAGAACAGACATAA \\
\hline KL034 & AAACAAACGACAGCATGCTGA \\
\hline KL035 & CTGGCCTCAGATCCTCGAA \\
\hline KL036 & CTCGCATAAAAAGCTGGAAGTG \\
\hline KL037 & ATCCAAAACCCTGGGCAAA \\
\hline KL038 & CCAAAAAGTAGTAAGGGAGAGGAT \\
\hline KL039 & ATAAACAGGGCCATATCGCATACA \\
\hline KL040 & TCATGTACTGTCCGGTGTGATT \\
\hline KL041 & ATCAAACTCCGTTTTTAGCCCC \\
\hline Mak31p1 & CCAAAGCGTCATGGACATCT \\
\hline Mak31p2 & AGGCCCATCATTCTACTACTGG \\
\hline MAT1 & ATTGCGACAAGGCTTCACCC \\
\hline MAT2 & CACATCACAGGTTTATTGGTTCCC \\
\hline MAT3-1 & ATGTCCTGACTTCTTTTGACGAGG \\
\hline MAT4-1 & ACGACCTATTTGTAACCGCACG \\
\hline MAT9 & GCCTCTATGTCCCCATCTTGTCTC \\
\hline MAT10 & GTGTTCCCGATTCAGTTTGACG \\
\hline MAT13 & TCAGGGTCTGGTGGAAGGAATG \\
\hline MAT14 & CAAAGGTGGCAGTTGTTGAACC \\
\hline MAT15 & CGTCTTCTCAGCGAACAACAGC \\
\hline MAT16 & GCAATAACCCACGGAAACACTG \\
\hline MAT19 & TCGTCGTCGCCATCATTTTC \\
\hline MAT20 & GCCCAAGTTTGAGAGAGGTTGC \\
\hline MATYAp4 & GATCTAAATAAATTCGTTTTCAATGATTAAAATAG \\
\hline MT101-Pho5orf +50 & CGCTTCTTTGGCCAATGC \\
\hline MT102-Pho5orf +100 & GGGTACCAATCTTGTCGAC \\
\hline RAD18p1 & TGTCATCGTTGGGACTGTCA \\
\hline
\end{tabular}


bioRxiv preprint doi: https://doi.org/10.1101/2019.12.17.877266; this version posted December 17, 2019. The copyright holder for this preprint (which was not certified by peer review) is the author/funder, who has granted bioRxiv a license to display the preprint in perpetuity. It is made available under aCC-BY-NC-ND 4.0 International license.

\begin{tabular}{ll} 
RAD18p2 & GAAACATAACCATCCATCCTTTCC \\
YCR026Cp1 & CACGCCTAGTTTCAGCTTGTTT \\
YCR026Cp2 & CTTCAAGACATAATCAACGACGC \\
YCR043Cp1 & CCAAGGAACTAATGATCTAAGCACA \\
YCR043Cp2 & ACCAGCAGTAATAAGTCGTCCTGA \\
YCR061Cp1 & GGAAAGACTGGCTCATCAAAAC \\
YCR061Cp2 & ACATTCTCAGAGAGAACCTCCA \\
\hline
\end{tabular}


bioRxiv preprint doi: https://doi.org/10.1101/2019.12.17.877266; this version posted December 17, 2019. The copyright holder for this preprint (which was not certified by peer review) is the author/funder, who has granted bioRxiv a license to display the preprint in perpetuity. It is made available under aCC-BY-NC-ND 4.0 International license.

\section{Supplemental Dataset}

\section{Dataset S1: $\gamma$-H2AX levels around MAT and RE}

$\gamma$-H2AX levels are shown in the Excel file "Dataset S1." $\gamma-\mathrm{H} 2 \mathrm{AX}$ levels were measured around the MAT locus for strains listed in Table S2. $\gamma$-H2AX measurements around RE were measured for strains yKL004 and yKL005. 


\section{References for Supplementary Information}

1. Arbona J-M, Herbert S, Fabre E, Zimmer C. Inferring the physical properties of yeast chromatin through Bayesian analysis of whole nucleus simulations. Genome Biol. 2017 Dec;18(1).

2. Hajjoul H, Mathon J, Ranchon H, Goiffon I, Mozziconacci J, Albert B, et al. Highthroughput chromatin motion tracking in living yeast reveals the flexibility of the fiber throughout the genome. Genome Res. 2013 Nov;23(11):1829-38.

3. Zhang Y, Dudko OK. First-Passage Processes in the Genome. Annu Rev Biophys. 2016 $05 ; 45: 117-34$.

4. Dekker J. Mapping in Vivo Chromatin Interactions in Yeast Suggests an Extended Chromatin Fiber with Regional Variation in Compaction. J Biol Chem. 2008 Dec 12;283(50):34532-40.

5. Gobbini E, Villa M, Gnugnoli M, Menin L, Clerici M, Longhese MP. Sae2 Function at DNA Double-Strand Breaks Is Bypassed by Dampening Tel1 or Rad53 Activity. JinksRobertson S, editor. PLOS Genet. 2015 Nov 19;11(11):e1005685.

6. Clerici M, Trovesi C, Galbiati A, Lucchini G, Longhese MP. Mec1/ATR regulates the generation of single-stranded DNA that attenuates Tel1/ATM signaling at DNA ends. EMBO J. 2014 Feb 3;33(3):198-216.

7. Berg H. Random Walks in Biology. New, expanded. Princeton University Press; 1993.

8. Singh AP, Galland R, Finch-Edmondson ML, Grenci G, Sibarita J-B, Studer V, et al. 3D Protein Dynamics in the Cell Nucleus. Biophys J. 2017 Jan 10;112(1):133-42.

9. Li J, Coïc E, Lee K, Lee C-S, Kim J-A, Wu Q, et al. Regulation of Budding Yeast MatingType Switching Donor Preference by the FHA Domain of Fkh1. Hunter N, editor. PLoS Genet. 2012 Apr 5;8(4):e1002630.

10. Avşaroğlu B, Bronk G, Li K, Haber JE, Kondev J. Chromosome-refolding model of mating-type switching in yeast. Proc Natl Acad Sci U S A. 2016 Nov 8;113(45):E6929-38.

11. Kozlov SV, Graham ME, Jakob B, Tobias F, Kijas AW, Tanuji M, et al. Autophosphorylation and ATM Activation ADDITIONAL SITES ADD TO THE COMPLEXITY. J Biol Chem. 2011 Mar 18;286(11):9107-19.

12. Nuebler J, Fudenberg G, Imakaev M, Abdennur N, Mirny LA. Chromatin organization by an interplay of loop extrusion and compartmental segregation. Proc Natl Acad Sci. 2018 Jul 17;115(29):E6697-706.

13. Arbona J-M, Herbert S, Fabre E, Zimmer C. Inferring the physical properties of yeast chromatin through Bayesian analysis of whole nucleus simulations. Genome Biol [Internet]. 2017 Dec [cited 2018 Jul 23];18(1). Available from: 
http://genomebiology.biomedcentral.com/articles/10.1186/s13059-017-1199-x

14. Brogaard K, Xi L, Wang J-P, Widom J. A map of nucleosome positions in yeast at basepair resolution. Nature. 2012 Jun;486(7404):496-501.

15. Kim J-A, Kruhlak M, Dotiwala F, Nussenzweig A, Haber JE. Heterochromatin is refractory to $\gamma$-H2AX modification in yeast and mammals. J Cell Biol. 2007 Jul 16;178(2):209-18.

16. Sivia DS, Skilling J. Data analysis: a Bayesian tutorial. 2nd ed. Oxford; New York: Oxford University Press; 2006. 246 p. (Oxford science publications). 